\title{
Structure-Reactivity Relationships in Rare-Earth Metal Carboxylate-Based Binary Ziegler-Type Catalysts
}

Andreas Fischbach, Franc Perdih, Eberhardt Herdtweck, and Reiner Anwander*

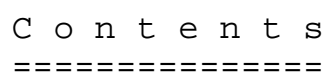

Table S1 - Crystal Data and Details of the Structure Determination for: Compound 1 $\mathbf{1 b}^{\prime}$

Table S2 - Final Coordinates and Equivalent Isotropic Thermal Parameters of the non-Hydrogen atoms for: Compound 1b'

Table S3 - Hydrogen Atom Positions and Isotropic Thermal Parameters for: Compound $\mathbf{1} \mathbf{b}^{\prime}$

Table S4 - (An)isotropic Thermal Parameters for: Compound 1b'

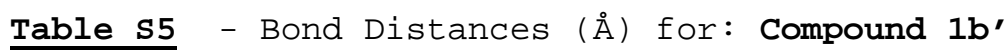

$\underline{\text { Table S6 }}$ - Bond Angles (Degrees) for: Compound 1b'

Table S7 - Crystal Data and Details of the Structure Determination for: Compound 1c'

Table S8 - Final Coordinates and Equivalent Isotropic Displacement Parameters of the non-Hydrogen atoms for: Compound $\mathbf{1} \mathbf{c}^{\prime}$

Table S9 - Hydrogen Atom Positions and Isotropic Displacement Parameters for: Compound $\mathbf{1} \mathbf{c}^{\prime}$

Table S10 - (An)isotropic Displacement Parameters for: Compound 1c'

Table S11 - Bond Distances (Angstrom) for: Compound 1c'

Table S12 - Bond Angles (Degrees) for: Compound 1c'

Table S13 - Crystal Data and Details of the Structure Determination for: Compound $9 \mathrm{~b}$

Table S14 - Final Coordinates and Equivalent Isotropic Displacement Parameters of the non-Hydrogen atoms for: Compound $\mathbf{9 b}$

Table S15 - Hydrogen Atom Positions and Isotropic Displacement Parameters for: Compound $\mathbf{9 b}$

Table S16 - (An)isotropic Displacement Parameters for: Compound 9b Table S17 - Bond Distances (Angstrom) for: Compound 9b

Table S18 - Bond Angles (Degrees) for: Compound 9b 
Table S19 - Crystal Data and Details of the Structure Determination for: Compound $9 \mathrm{c}$

Table S20 - Final Coordinates and Equivalent Isotropic Displacement Parameters of the non-Hydrogen atoms for: Compound 9c

Table S21 - Hydrogen Atom Positions and Isotropic Displacement Parameters for: Compound 9c

Table S22 - (An)isotropic Displacement Parameters for: Compound 9c

Table S23 - Bond Distances (Angstrom) for: Compound 9c

Table S24 - Bond Angles (Degrees) for: Compound 9c

Table s25 - Crystal Data and Details of the Structure Determination for: Compound 10b

Table s26 - Final Coordinates and Equivalent Isotropic Displacement Parameters of the non-Hydrogen atoms for: Compound 10b

Table S27 - Hydrogen Atom Positions and Isotropic Displacement Parameters for: Compound 10b

Table S28 - (An)isotropic Displacement Parameters for: Compound 10b

Table S29 - Bond Distances (Angstrom) for: Compound 10b

Table S30 - Bond Angles (Degrees) for: Compound 10b

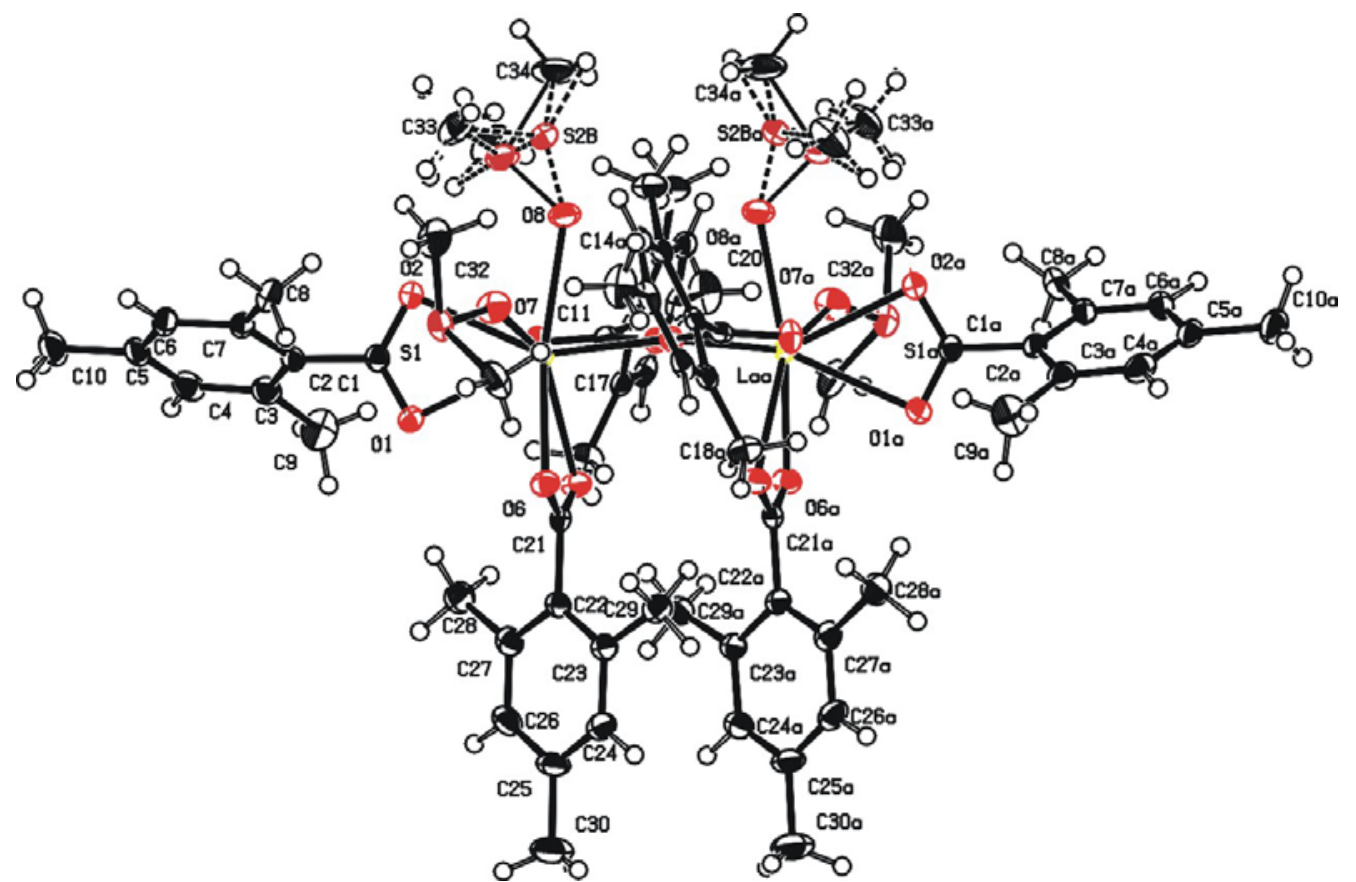

Molecular structure of $\left[\mathrm{La}\left(\mathrm{O}_{2} \mathrm{CC}_{6} \mathrm{H}_{2} \mathrm{Me}_{3}-2,4,6\right)_{2}\left(\mu-\mathrm{O}_{2} \mathrm{CC}_{6} \mathrm{H}_{2} \mathrm{Me}_{3}-2,4,6\right)(\mathrm{DMSO})_{2}\right]_{2}\left(\mathbf{1 b}^{\prime}\right)$ 
Table S1 - Crystal Data and Details of the Structure Determination for: Compound 1 $\mathbf{b}^{\prime}$

Crystal Data
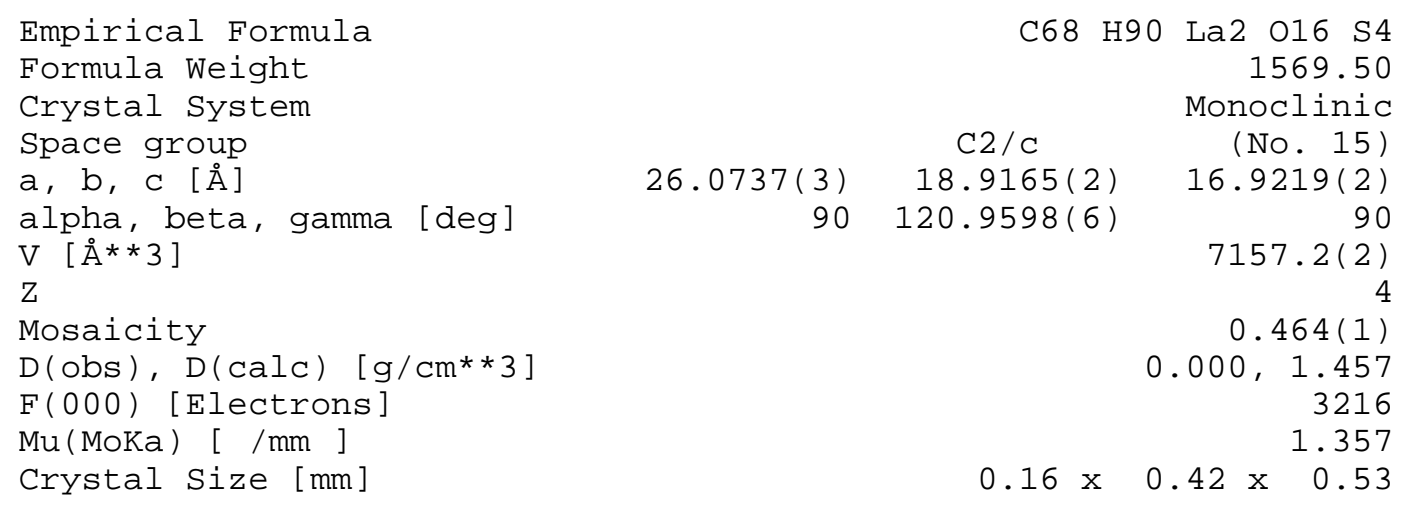

Data Collection

Temperature (K)

Drehanode

Theta Min-Max [Deg]

Kollimator [Nr.;mm]

Device

Drehanode $50 / 60 \quad$ MoKa 0.71073

Scan Characteristics set 1 [mm; Deg; Deg]:

$2.15, \quad 25.34$ $0540.353 ; 0.61$ Nonius Kappa CCD $\mathrm{Dx}=40 ;$ Phi movement mode; Delta-Phi $=1$

Exposure time [s] $x$ repetition $20 \times 2$

Data set

Resolution [min; max]

Completeness of the data set

Tot., Uniq. Data, R(int)

Observed data $[\mathrm{I}>2.0$ sigma(I)]

$-31: 31 ;-22: 22 ;-20: 20$

$20.40 ; \quad 0.83$

$18159, \quad 6433, \quad 0.024$

Refinement

Nref, Npar

R1 [I > $2.0 \operatorname{sigma(I)]}$

$6433, \quad 581$

5786

$\mathrm{R} 1, \mathrm{WR}, \mathrm{S}$ [all data]

Weighting Scheme : $w=1 /\left[\operatorname{sigma}^{2}\left(\mathrm{Fo}^{2}\right)+(\mathrm{a} * \mathrm{P})^{2}+\mathrm{b} * \mathrm{P}\right]$

with $\mathrm{a}: 0.0144 ; \mathrm{b}: 14.5714 ; \mathrm{P}:\left[\operatorname{Maximum}\left(\odot\right.\right.$ or $\left.\mathrm{Fo}^{2}\right)+2 * \mathrm{Fc}^{2}$ ]/3

Max. and Av. Shift/Error

$0.00,0.00$

Min. and Max. resd. dens. [e/Ang $\left.{ }^{3}\right]$

$-0.44,0.62$

Table S2 - Final Coordinates and Equivalent Isotropic Thermal Parameters of the non-Hydrogen atoms for Compound $\mathbf{1 b}$ '

\begin{tabular}{|c|c|c|c|c|}
\hline Atom & $\mathrm{X}$ & $\mathrm{y}$ & Z & $\mathrm{U}(\mathrm{eq}) \quad\left[\AA^{2}\right]$ \\
\hline--- & --- & -- & -- & ----- \\
\hline La & $0.071261(5)$ & $0.331900(6)$ & ๑.391703(8) & ๑. $0155(1)$ \\
\hline S1 & $0.23707(3)$ & $0.35931(3)$ & $0.48719(4)$ & $0.0242(2)$ \\
\hline 01 & $0.12569(7)$ & $0.27363(8)$ & $0.55437(10)$ & $0.0240(5)$ \\
\hline 02 & $0.11459(7)$ & ๑. 38948(8) & $0.55394(10)$ & $0.0264(5)$ \\
\hline 03 & $-0.01574(7)$ & $0.34372(8)$ & $0.42091(10)$ & $0.0246(5)$ \\
\hline 04 & $\odot .04800(6)$ & $0.34368(7)$ & $0.22808(10)$ & ๑. $0181(5$ \\
\hline 05 & $0.02607(6)$ & $0.21071(8)$ & $0.36204(11)$ & $0.0232(5)$ \\
\hline 06 & $0.11676(7)$ & $0.21109(8)$ & $0.38133(10)$ & $0.0215(5)$ \\
\hline 07 & $0.17079(7)$ & $0.37549(9)$ & $0.43259(12)$ & $0.0300(5)$ \\
\hline 08 & ०. $04824(8)$ & $0.46114(8)$ & $\odot .36605(12)$ & ๑. .0358(5) \\
\hline C1 & $0.13235(10)$ & $0.33108(11)$ & $\odot .59589(15)$ & $0.0192(7$ \\
\hline C2 & $0.16227(10)$ & $0.33203(11)$ & ๑. 69976(15) & $0.0205(7$ \\
\hline
\end{tabular}


Table S2 (cont.) - Final Coordinates and Equivalent Isotropic Thermal Parameters of the non-Hydrogen atoms for Compound 16'

\begin{tabular}{|c|c|c|c|c|}
\hline com & $x$ & y & z & $U(e q)\left[\AA^{2}\right]$ \\
\hline & & & & \\
\hline C3 & $0.13313(11)$ & $0.30343(13)$ & $229(16)$ & $\odot$ \\
\hline 4 & $\odot .16082(12)$ & $0.30902(13)$ & $\odot .83821(17)$ & $0.0304(8$ \\
\hline C5 & $0.21606(12)$ & $0.34104(12)$ & $\odot .89176(16)$ & $0.0285(8)$ \\
\hline$C 6$ & $0.24445(11)$ & $0.36812(13)$ & $\odot .84786(16)$ & $0.0255(7$ \\
\hline $\mathrm{C} 7$ & $0.21864(10)$ & $0.36370(11)$ & $0.75240(15)$ & $0.0208(7)$ \\
\hline $\mathrm{c} 8$ & $0.25227(12)$ & $\odot .39019(14)$ & $0.70821(18)$ & $0.0267(8)$ \\
\hline $\mathrm{Cg}$ & $0.07382(14)$ & $\odot .26656(19)$ & $0.6873(2)$ & \\
\hline C10 & $\odot .24624(17)$ & $\odot .34586(18)$ & $\odot .99525(19)$ & $0.0404(10)$ \\
\hline C11 & $0.05740(10)$ & $0.34862(11)$ & $0.33897(15)$ & $0.0176(6)$ \\
\hline $\mathrm{C} 12$ & $-0.12010(9)$ & $0.36043(11)$ & $0.31938(14)$ & $0.0172(6)$ \\
\hline 13 & $-0.14890(10)$ & $0.42502(11)$ & $0.28407(14)$ & \\
\hline C14 & $-0.20633(10)$ & $0.43389(13)$ & $0.26959(15)$ & 0 \\
\hline 15 & $-0.23553(10)$ & $\odot .38075(13)$ & $\odot .28772(15)$ & $\odot .0238(7)$ \\
\hline C16 & -0.20556 & $\odot .31747(13)$ & $0.32353(15)$ & $0.0227(7)$ \\
\hline C17 & $-0.14770(10)$ & $0.30641(12)$ & $0.34105(14)$ & $0.0198(7)$ \\
\hline $\mathrm{C} 18$ & $-0.11560(13)$ & $0.23763(14)$ & $0.3820(2)$ & $0.0306(8)$ \\
\hline C19 & $-0.11956(13)$ & $0.48524(13)$ & $0.2633(2)$ & $0.0295(8)$ \\
\hline $\mathrm{C} 20$ & $-0.29919(13)$ & $0.3906(2)$ & $0.2653(2)$ & $0.0383(10$ \\
\hline $\mathrm{C} 21$ & 0.0717 & 0.17 & $0.37265(14)$ & $0.0180(6)$ \\
\hline 22 & 0722 & 0.09880 & 0.37767 & $0.0200(7)$ \\
\hline $\mathrm{C} 23$ & & & 0.315 & $0.0214(7)$ \\
\hline C24 & $.08007(10)$ & -0.015 & 0.321 & $0.0268(7)$ \\
\hline $\mathrm{C} 25$ & $0.07227(11)$ & $-0.04948(12)$ & $0.38754(17)$ & $0.0308(8)$ \\
\hline C26 & ค $06499(11)$ & -0.00789 ( & $\odot .44897(17)$ & ค $0297(8)$ \\
\hline $\mathrm{C} 27$ & $\odot .06389(10)$ & $\odot .06572(12)$ & $0.44465(16)$ & $0.0249(7)$ \\
\hline $\mathrm{C} 28$ & $\odot .05429(13)$ & $0.10789(15)$ & $\odot .51153(19)$ & $\odot .0315(9)$ \\
\hline C29 & $\odot .08970(12)$ & $\odot .09262(14)$ & $\odot .24326(18)$ & $0.0255(8)$ \\
\hline C30 & 00700 & -0.129 & $0.3917(3)$ & $0.0487(11$ \\
\hline C31 & 0.250 & 0.30 & 0.411 & 0.0379 \\
\hline C32 & 0.271 & 0.4 & 0.484 & 0.0389 \\
\hline * $\mathrm{S} 2 \mathrm{~A}$ & 0.0579 & 0.516 & 0.44455 (11) & 0.0324 \\
\hline${ }^{*} \mathrm{C} 33$ & $\odot .13234(12)$ & $0.54379(15)$ & $0.4868(2)$ & $0.0406(9)$ \\
\hline${ }^{*} \mathrm{C} 34$ & $0.0246(2)$ & $0.5952(2)$ & $0.3814(4)$ & $0.0686(1 \varepsilon$ \\
\hline${ }^{*} \mathrm{~S} 2 \mathrm{E}$ & 0.0524 & $0.53476(11)$ & $0.39022(19)$ & $0.0276(7)$ \\
\hline${ }^{*} \mathrm{C} 36$ & 0.0248 & $0.5284(4)$ & $0.4673(6)$ & \\
\hline${ }^{*} \mathrm{C} 35$ & $0.13234(12)$ & $\odot .54379(15)$ & $\odot .4868(2)$ & $0.0406(9)$ \\
\hline
\end{tabular}

$U(e q)=1 / 3$ of the trace of the orthogonalized $U$

Starred Atom sites have a S.O.F less than 1.0

Table S3 - Hydrogen Atom Positions and Isotropic Thermal Parameters for Compound 1b'

$\begin{array}{lcccc}\text { Atom } & x & y & z & U(e q)\left[\AA^{2}\right] \\ ---- & --- & --- & -- & --.-\ldots \\ \text { H41 } & 0.1400(12) & 0.2896(15) & 0.8665(19) & 0.043(8) \\ \text { H61 } & 0.2842(12) & 0.3900(13) & 0.8867(17) & 0.029(7) \\ \text { H81 } & 0.2288(12) & 0.4200(14) & 0.6571(19) & 0.036(7) \\ \text { H82 } & 0.2644(13) & 0.3508(15) & 0.6848(19) & 0.044(8) \\ \text { H83 } & 0.2869(13) & 0.4157(14) & 0.7515(19) & 0.038(7) \\ \text { H91 } & 0.0503(14) & 0.2831(16) & 0.630(2) & 0.048(9) \\ \text { H92 } & 0.0516(14) & 0.2687(16 & 0.716(2) & 0.053(9) \\ \text { H93 } & 0.0774(18) & 0.215(2) & 0.676(3) & 0.097(14) \\ \text { H101 } & 0.2581(16) & 0.393(2) & 1.018(2) & 0.079(12) \\ \text { H102 } & 0.2786(16) & 0.3168(18) & 1.024(2) & 0.060(10)\end{array}$


Table s3 (cont.)

$\begin{array}{lc}\text { Atom } & \text { X } \\ ---- & -- \\ \text { H103 } & 0.2215(17) \\ \text { H141 } & -0.2234(11) \\ \text { H161 } & -0.2256(11) \\ \text { H181 } & -0.0941(13) \\ \text { H182 } & -0.1409(13) \\ \text { H183 } & -0.0871(15) \\ \text { H191 } & -0.0847(15) \\ \text { H192 } & -0.1152(15) \\ \text { H193 } & -0.1447(18) \\ \text { H201 } & -0.3124(16) \\ \text { H202 } & -0.3234(19) \\ \text { H203 } & -0.3029(17) \\ \text { H241 } & 0.0843(11) \\ \text { H261 } & 0.0589(11) \\ \text { H281 } & 0.0809(12) \\ \text { H282 } & 0.0601(12) \\ \text { H283 } & 0.0136(14) \\ \text { H291 } & 0.0597(11) \\ \text { H292 } & 0.0884(12) \\ \text { H293 } & 0.1267(13) \\ \text { H301 } & 0.081(2) \\ \text { H302 } & 0.036(2) \\ \text { H303 } & 0.097(2) \\ \text { H311 } & 0.2256(14) \\ \text { H312 } & 0.2912(15) \\ \text { H313 } & 0.2370(14) \\ \text { H321 } & 0.2671(14) \\ \text { H322 } & 0.2496(13) \\ \text { H323 } & 0.3140(16) \\ { }^{*} H 331 & 0.1598 \\ { }^{*} H 332 & 0.1414 \\ { }^{*} H 333 & 0.1371 \\ { }^{*} H 341 & -0.0190 \\ { }^{*} H 342 & 0.0376 \\ { }^{*} H 343 & 0.0369 \\ { }^{*} H 351 & 0.1574 \\ { }^{*} H 352 & 0.1462 \\ { }^{*} H 353 & 0.1350 \\ { }^{*} H 361 & -0.0182 \\ { }^{*} H 362 & 0.0319 \\ { }^{*} H 363 & 0.0456 \\ & \end{array}$

- Hydrogen Atom Positions and Isotropic Thermal Parameters for Compound 1 $\mathbf{1 b}^{\prime}$

\begin{tabular}{|c|c|c|}
\hline$y$ & Z & $\mathrm{U}(\mathrm{eq}) \quad\left[\AA^{2}\right]$ \\
\hline- & -- & \\
\hline ๑. 3315(18) & 1.016(2) & $0.070(12)$ \\
\hline $0.4736(13)$ & ๑. 2471(16) & $0.021(6)$ \\
\hline ๑.2799(13) & $0.3357(16)$ & $0.027(6)$ \\
\hline ๑.2217(16) & $0.354(2)$ & $0.051(9)$ \\
\hline ๑. 2019(16) & ๑. $3817(19)$ & $0.046(8)$ \\
\hline ๑.2449(18) & $0.444(3)$ & $0.066(11)$ \\
\hline $0.4732(16)$ & $0.268(2)$ & $0.056(9)$ \\
\hline ๑.5237(19) & $0.301(2)$ & $0.070(11)$ \\
\hline $0.505(2)$ & $0.207(3)$ & $0.093(14)$ \\
\hline ๑.3583(19) & $0.293(2)$ & $0.069(11)$ \\
\hline $0.391(2)$ & $0.210(3)$ & $0.092(15)$ \\
\hline$\odot .431(2)$ & $0.287(3)$ & $0.085(14)$ \\
\hline$-0.0424(13)$ & $0.2778(17)$ & $0.027(6)$ \\
\hline$-0.0282(14)$ & $0.4943(18)$ & $0.032(7)$ \\
\hline $0.1474(14)$ & $0.5376(18)$ & $0.032(7)$ \\
\hline $0.0774(14)$ & $0.5633(19)$ & $0.038(7)$ \\
\hline $0.1287(16)$ & $0.481(2)$ & $0.050(9)$ \\
\hline $0.1283(14)$ & ๑. 2086(17) & $0.029(7)$ \\
\hline ๑.0569(15) & $0.2011(19)$ & $0.039(7)$ \\
\hline $0.1172(14)$ & ๑. 2702(18) & $0.035(7)$ \\
\hline$-0.148(2)$ & $\odot .355(3)$ & $0.098(16)$ \\
\hline$-0.145(3)$ & $0.372(4)$ & $0.13(2)$ \\
\hline$-0.148(3)$ & $0.450(4)$ & $0.126(19)$ \\
\hline $0.2634(18)$ & $0.399(2)$ & $0.058(10)$ \\
\hline $0.3000(16)$ & $0.442(2)$ & $0.050(9)$ \\
\hline ๑.3319(15) & $\odot .356(2)$ & $0.048(9)$ \\
\hline $0.4715(17)$ & $0.525(2)$ & $0.055(9)$ \\
\hline $0.4570(16)$ & $0.420(2)$ & $0.051(9)$ \\
\hline ๑. $4297(18)$ & $0.507(2)$ & $0.070(11)$ \\
\hline 0.5050 & 0.5210 & 0.049 \\
\hline$\odot .5844$ & 0.5278 & 0.049 \\
\hline 0.5573 & 0.4351 & 0.049 \\
\hline 0.5906 & 0.3483 & 0.082 \\
\hline 0.6032 & 0.3372 & 0.082 \\
\hline 0.6352 & $\odot .4241$ & 0.082 \\
\hline$\odot .5569$ & 0.4616 & 0.049 \\
\hline ๑. 4987 & 0.5196 & 0.049 \\
\hline ๑.5805 & ๑. 5297 & ๑. 049 \\
\hline 0.5185 & 0.4320 & 0.046 \\
\hline 0.5731 & 0.5006 & 0.046 \\
\hline$\odot .4900$ & 0.5114 & 0.046 \\
\hline
\end{tabular}

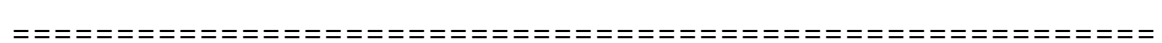

The Temperature Factor has the Form of $\operatorname{Exp}(-\mathrm{T})$ Where

$\mathrm{T}=8^{*}\left(\mathrm{Pi}^{* *} 2\right)^{*} \mathrm{U}^{*}(\operatorname{Sin}($ Theta $) /$ Lambda $){ }^{*} 2$ for Isotropic Atoms

Table S4 - (An)isotropic Thermal Parameters for Compound 1b'

\begin{tabular}{|c|c|c|c|c|c|c|}
\hline Atom & $U(1,1)$ & $U(2,2)$ & $U(3,3)$ & $U(2,3)$ & $U(1,3)$ & $\mathrm{U}(1,2)$ \\
\hline _ & $-\cdots$ & ---- & 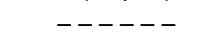 & $\ldots$ & & $\ldots$ \\
\hline$-a$ & 0.013 & $.0150(1)$ & 012 & $-0.00 \odot$ & 0.00 & $-\odot$ \\
\hline 1 & $0.0223(3)$ & $0.0305(3)$ & .018 & $0.0022(2)$ & $0.0097(3)$ & $\odot$ \\
\hline 11 & $0.0284(9)$ & $0.0197(8)$ & $0.0193(8)$ & $-0.0027(7)$ & $0.0089(7)$ & $\odot .0 \odot$ \\
\hline 02 & $0.0319(9)$ & $\odot .0210(9)$ & $0.0173(8)$ & $-0.0012(7)$ & $0.0062(7)$ & $0.0004(7$ \\
\hline 03 & 0. .0194( 8$)$ & ๑.0342(9) & 0.0161(8) & $-\odot .0010(7)$ & $0.0063(7)$ & $\odot . \odot \odot 2 \odot(7$ \\
\hline 04 & $180(8)$ & $0.0184(8)$ & $0.0168(8)$ & $0.0007(6)$ & $0.0082(7)$ & -0.0025 \\
\hline 5 & ๑.0150(8) & ๑.0188(8) & $0.0302(9)$ & $-0.0017(7)$ & $0.0077(7)$ & 0.0001 \\
\hline
\end{tabular}




\section{Table S4 (cont.)}

- (An)isotropic Thermal Parameters for Compound 1 $\mathbf{b}^{\prime}$

\begin{tabular}{|c|c|c|c|c|c|c|}
\hline Atom & $\mathrm{U}(1,1)$ & $U(2,2)$ & $\mathrm{U}(3,3)$ & $U(2,3)$ & $U(1,3)$ & $U(1,2)$ \\
\hline & & & & & & \\
\hline & $82(8)$ & $0(0)$ & & J & (1) & 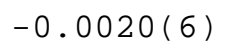 \\
\hline 7 & (8) & $0308(9)$ & $(10)$ & $2(8)$ & 0128 ( 8) & $3(7)$ \\
\hline 08 & ๑. .0299(10) & 0168 ( 8 ) & $372(10)$ & ๑. .0020( 8$)$ & $0.0005(8)$ & $-0.0003(7)$ \\
\hline 1 & 151(11) & $.0222(12)$ & $180(11)$ & ๑.0006(9) & $0.0069(10)$ & $0.0021(9)$ \\
\hline 2 & $237(12)$ & $0.0180(11)$ & $0172(11)$ & $0.0010(9)$ & $0.0087(10)$ & $0.0067(9)$ \\
\hline 3 & $0299(13)$ & $0.0238(12)$ & $0.0271(13)$ & $0.0018(10)$ & $0.0156(11)$ & $0.0040(10)$ \\
\hline C4 & $0.0443(16)$ & $0.0268(13)$ & $0.0293(14)$ & ๑.0064(11) & $0.0255(13)$ & $0.0083(12)$ \\
\hline 5 & $399(15)$ & ๑. 0244(12) & $0.0193(12)$ & ๑. . $0029(10)$ & 0.0139(12) & $0.0144(11)$ \\
\hline 6 & $250(13)$ & $0.0247(12)$ & $200(12)$ & $-0.0036(10)$ & $0.0067(11)$ & $0.0100(10)$ \\
\hline 57 & $212(12)$ & $0.0199(11)$ & $184(11)$ & $-0.0022(9)$ & $0.0081(10)$ & $0.0077(9)$ \\
\hline 8 & $219(13)$ & $0.0336(14)$ & $214(13)$ & $-0.0080(12)$ & ๑. . $0089(11)$ & $-0.0007(11)$ \\
\hline 59 & $387(17)$ & $0.054(2)$ & $415(18)$ & $-0.0043(16)$ & $0.0263(16)$ & $-0.0109(15)$ \\
\hline C10 & $58(2)$ & $0.0385(17)$ & $0.0224(14)$ & $0.0053(13)$ & $0.0191(15)$ & $0.0163(16)$ \\
\hline 1 & $194(12)$ & $0.0129(10)$ & $0.0195(11)$ & $-0.0016(9)$ & 92 & $03(9)$ \\
\hline 2 & $163(11)$ & 0.01 & 0.012 & -0.00 & $7(9)$ & $-0.0007(9)$ \\
\hline 13 & 198(11) & $0.0197(11)$ & 0.016 & $-0.0054(9)$ & $76(10$ & $-0.0012(9)$ \\
\hline C14 & $211(12)$ & $0.0224(12)$ & $192(12)$ & $-0.0034(10)$ & $0.0075(10)$ & $0.0046(10)$ \\
\hline C15 & $0.0184(12)$ & $0.0366(13)$ & 153(11) & $-0.0075(10)$ & $0.0078(10)$ & $-0.0019(10)$ \\
\hline C16 & $0.0243(12)$ & $0.0294(13)$ & $158(11)$ & $-0.0067(10)$ & $0.0114(10)$ & $-0.0078(10)$ \\
\hline C17 & $236(12)$ & $0.0213(11)$ & 0.014 & $-0.0044(9)$ & $88(10)$ & $-0.0028(9)$ \\
\hline 18 & $46(15)$ & 0.02 & 0.03 & $2(12)$ & 0.0211(14) & $0.0002(11)$ \\
\hline C19 & $9(14)$ & $0.0189(13)$ & $7(16)$ & ๑. . $0010(12)$ & $0.0160(13)$ & $0.0001(11)$ \\
\hline C20 & $219(14)$ & $\odot .060(2)$ & $0.0343(17)$ & $\odot .0030(15)$ & $0.0154(14)$ & $0.0038(14)$ \\
\hline C21 & $158(11)$ & $0.0193(11)$ & $136(10)$ & $-0.0010(9)$ & ๑.0०38(9) & $-0.0005(9)$ \\
\hline C22 & $0.0102(11)$ & $0.0193(11)$ & $0.0223(12)$ & ๑. .0017(9) & $0.0025(10)$ & $0.0010(8)$ \\
\hline $\mathrm{C} 23$ & $0.0109(11)$ & $0.0204(11)$ & $0.0250(12)$ & $-0.0004(9)$ & $0.0035(10)$ & $0.0019(9)$ \\
\hline C24 & $90(12)$ & $0.0231(12)$ & 0.02 & $-0.0041(11)$ & $0.0065(11)$ & $3(10)$ \\
\hline $\mathrm{C} 25$ & (13) & 0.0 & 0.039 & 0.003 & ๑. $0094(12$ & -0.00 \\
\hline C26 & $274(13)$ & $0.0280(13)$ & $0.0292(13)$ & $0.0090(11)$ & $0.0113(12$ & $-0.0019(11)$ \\
\hline C27 & $0.0165(12)$ & $0.0270(13)$ & $0.0252(12)$ & $0.0033(10)$ & $0.0064(10$ & -0.00 \\
\hline C28 & ๑.0358(16) & $0.0313(15$ & $0.0284(14)$ & $0.0007(12)$ & ๑. .0173(13 & $-0.0029(13)$ \\
\hline C29 & $\odot .0229(13)$ & $0.0246(13$ & $0.0282(13$ & $-0.0024(11)$ & $0.0125(11$ & $0.0007(11)$ \\
\hline C30 & $7(2)$ & ๑. .0206(15 & $0.061(2)$ & ๑.0042(15) & $0.025(2)$ & $-0.0015(14)$ \\
\hline C31 & $6(19)$ & 0.044 & $0.0255(14)$ & $0.0104(13)$ & $0.0210(14)$ & 0.024 \\
\hline C32 & $\odot .0230(15)$ & $0.0482(18)$ & $0.0374(17$ & $-0.0041(14)$ & 0.0098(14) & $-0.0099(13)$ \\
\hline S2A & $0.0311(6)$ & ๑. .0182(5) & $0.0379(10$ & $-0.0038(5)$ & ๑.0105(5) & $0.0014(4)$ \\
\hline C33 & $0.0335(15)$ & $0.0363(15)$ & $0.0434(16$ & $-0.0152(13)$ & $0.0137(14$ & $-0.0122(12)$ \\
\hline C34 & $45(3)$ & $0.019(2)$ & $0.082(4)$ & $0.000(2)$ & $-0.010(3)$ & $0.008(2)$ \\
\hline S2 & $0(12)$ & 0.01 & 0.022 & $-0.0035(10)$ & $0.0118(10)$ & $0.0017(8)$ \\
\hline C36 & $0.035(5)$ & $0.049(5)$ & $0.047(5)$ & $-0.020(4)$ & $0.033(4)$ & $0.000(4)$ \\
\hline C35 & $0.0335(15)$ & $0.0363(15)$ & $0.0434(16)$ & $-0.0152(13)$ & $0.0137(14)$ & $-0.0122(12)$ \\
\hline
\end{tabular}

The Temperature Factor has the Form of $\operatorname{Exp}(-\mathrm{T})$ Where

$\mathrm{T}=8^{*}\left(\mathrm{Pi} *{ }^{*} 2\right) \mathrm{U}^{*}(\operatorname{Sin}($ Theta)/Lambda $) * * 2$ for Isotropic Atoms

$T=2^{*}\left(P i *{ }^{*} 2\right){ }^{*} \operatorname{Sumij}\left(h(i){ }^{*} h(j){ }^{*} U(i, j){ }^{*} \operatorname{Astar}(i){ }^{*} A s t a r(j)\right)$, for Anisotropic Atoms. Astar(i) are Reciprocal Axial Lengths and h(i) are the Reflection Indices.

$\underline{\text { Table S5 }}$ - Bond Distances $(\AA)$ for Compound 16'

$\begin{array}{llllll}\mathrm{La} & -01 & 2.6051(15) & \mathrm{C} 4 & -\mathrm{H} 41 & 0.96(3) \\ \mathrm{La} & -02 & 2.6093(15) & \mathrm{C} 6 & -\mathrm{H} 61 & 0.99(3) \\ \mathrm{La} & -03 & 2.568(2) & \mathrm{C} 8 & -\mathrm{H} 82 & 0.97(3) \\ \mathrm{La} & -04 & 2.5211(15) & \mathrm{C} 8 & -\mathrm{H} 83 & 0.95(3) \\ \mathrm{La} & -05 & 2.5075(16) & \mathrm{C} 8 & -\mathrm{H} 81 & 0.95(3) \\ \mathrm{La} & -06 & 2.6220(17) & \mathrm{C} 9 & -\mathrm{H} 91 & 0.90(3) \\ \mathrm{La} & -07 & 2.459(2) & \mathrm{C} 9 & -\mathrm{H} 92 & 0.93(4) \\ \mathrm{La} & -08 & 2.5015(15) & \mathrm{C} 9 & -\mathrm{H} 93 & 1.01(4) \\ \mathrm{La} & -\mathrm{C} 21 & 2.923(2) & \mathrm{C} 10 & -\mathrm{H} 101 & 0.96(4)\end{array}$


Table S5 (cont.) - Bond Distances ( $\AA$ ) for Compound 1b'

\begin{tabular}{|c|c|c|c|c|c|}
\hline La & $-04 \_a$ & $2.7097(17)$ & C10 & $-\mathrm{H} 102$ & $0.91(4)$ \\
\hline S1 & -07 & $1.514(2)$ & $\mathrm{C} 10$ & $-\mathrm{H} 103$ & $0.92(5)$ \\
\hline S1 & $-C 31$ & $1.779(4)$ & C14 & $-\mathrm{H} 141$ & $0.86(3)$ \\
\hline S1 & $-\mathrm{C} 32$ & $1.777(3)$ & C16 & - H161 & $0.97(3)$ \\
\hline S2A & -08 & $1.601(2)$ & C18 & - H181 & $0.95(4)$ \\
\hline S2A & - C33 & $1.766(4)$ & $\mathrm{C} 18$ & - H183 & $0.93(4)$ \\
\hline S2A & $-\mathrm{C} 34$ & $1.784(5)$ & C18 & - H182 & $0.94(3)$ \\
\hline S2A & $-C 35$ & $1.766(4)$ & C19 & - H191 & ๑.90(4) \\
\hline S2B & $-C 35$ & $1.881(4)$ & C19 & - H193 & $0.91(4)$ \\
\hline S2B & $-\mathrm{C} 36$ & $1.789(11)$ & C19 & - H192 & $0.94(3)$ \\
\hline S2B & - C33 & $1.881(4)$ & $\mathrm{C} 20$ & $-\mathrm{H} 202$ & $0.82(4)$ \\
\hline S2B & -08 & $1.440(3)$ & $\mathrm{C} 20$ & $-\mathrm{H} 203$ & $0.87(4)$ \\
\hline 01 & $-C 1$ & $1.257(3)$ & $\mathrm{C} 20$ & - H201 & $0.94(4)$ \\
\hline 02 & $-C 1$ & $1.265(3)$ & $\mathrm{C} 24$ & $-\mathrm{H} 241$ & $0.95(3)$ \\
\hline 03 & $-C 11$ & $1.250(3)$ & $\mathrm{C} 26$ & - H261 & $0.94(3)$ \\
\hline 04 & $-C 11 \_a$ & $1.282(3)$ & $\mathrm{C} 28$ & $-H 281$ & $0.96(3)$ \\
\hline 05 & $-C 21$ & $1.266(3)$ & C28 & $-\mathrm{H} 282$ & $0.99(3)$ \\
\hline 06 & $-C 21$ & $1.268(3)$ & $\mathrm{C} 28$ & $-\mathrm{H} 283$ & $0.99(4)$ \\
\hline C1 & $-\mathrm{C} 2$ & $1.512(3)$ & C29 & $-\mathrm{H} 292$ & $0.97(3)$ \\
\hline C2 & $-\mathrm{C3}$ & $1.397(4)$ & C29 & - H293 & $0.95(3)$ \\
\hline $\mathrm{C} 2$ & $-C 7$ & $1.402(4)$ & $\mathrm{C} 29$ & $-H 291$ & $0.97(3)$ \\
\hline C3 & $-C 9$ & $1.504(5)$ & C30 & $-\mathrm{H} 302$ & $0.85(6)$ \\
\hline C3 & $-\mathrm{C} 4$ & $1.400(3)$ & C30 & - H303 & $0.93(6)$ \\
\hline C4 & $-\mathrm{C5}$ & $1.386(4)$ & C30 & - H301 & $0.87(5)$ \\
\hline C5 & $-\mathrm{C} 10$ & $1.509(4)$ & C31 & - H312 & $0.93(4)$ \\
\hline C5 & $-\mathrm{C6}$ & $1.389(4)$ & C31 & - H313 & $0.95(3)$ \\
\hline $\mathrm{C} 6$ & $-C 7$ & $1.397(3)$ & C31 & $-H 311$ & $0.99(4)$ \\
\hline C7 & $-\mathrm{C} 8$ & $1.501(4)$ & C32 & -H321 & $0.96(3)$ \\
\hline C11 & $-\mathrm{C} 12$ & $1.508(4)$ & C32 & $-H 322$ & $1.00(3)$ \\
\hline C12 & $-\mathrm{C} 13$ & $1.397(3)$ & C32 & $-H 323$ & $0.98(4)$ \\
\hline C12 & $-\mathrm{C} 17$ & $1.403(3)$ & & & \\
\hline C13 & $-\mathrm{C} 14$ & $1.397(4)$ & C33 & -H331 & 0.98 \\
\hline C13 & - C19 & $1.511(4)$ & C33 & -H332 & 0.98 \\
\hline C14 & - C15 & $1.387(4)$ & C33 & $-\mathrm{H} 333$ & 0.98 \\
\hline C15 & $-\mathrm{C} 20$ & $1.512(5)$ & C34 & $-H 342$ & 0.98 \\
\hline C15 & - C16 & $1.387(4)$ & C34 & $-H 343$ & 0.98 \\
\hline C16 & $-\mathrm{C} 17$ & $1.396(4)$ & C34 & - H341 & 0.98 \\
\hline C17 & $-\mathrm{C} 18$ & $1.509(4)$ & C35 & $-H 351$ & 0.98 \\
\hline C21 & $-C 22$ & $1.507(3)$ & C35 & $-H 352$ & 0.98 \\
\hline $\mathrm{C} 22$ & $-\mathrm{C} 27$ & $1.407(3)$ & C35 & $-H 353$ & 0.98 \\
\hline C22 & $-\mathrm{C} 23$ & $1.406(3)$ & C36 & - H361 & 0.98 \\
\hline $\mathrm{C} 23$ & $-C 24$ & $1.395(3)$ & C36 & $-H 362$ & 0.98 \\
\hline C23 & $-C 29$ & $1.507(4)$ & C36 & $-H 363$ & 0.98 \\
\hline C24 & $-C 25$ & $1.392(4)$ & & & \\
\hline C25 & $-C 26$ & $1.392(4)$ & & & \\
\hline C25 & $-\mathrm{C} 30$ & $1.509(4)$ & & & \\
\hline C26 & $-C 27$ & $1.394(3)$ & & & \\
\hline $\mathrm{C} 27$ & $-C 28$ & $1.507(4)$ & & & \\
\hline
\end{tabular}

Table S6 - Bond Angles (Degrees) for Compound 1b'

\begin{tabular}{|c|c|c|c|c|c|c|c|}
\hline 01 & $-L a$ & -02 & $50.15(5)$ & C25 & $-C 26$ & $-C 27$ & $122.2(2)$ \\
\hline 01 & $-L a$ & -03 & $83.60(6)$ & $\mathrm{C} 22$ & $-C 27$ & $-C 26$ & $118.6(2)$ \\
\hline 01 & - La & -04 & $152.92(5)$ & C26 & $-C 27$ & $-C 28$ & $119.8(2)$ \\
\hline 01 & - La & -05 & $76.65(5)$ & $\mathrm{C} 22$ & $-C 27$ & $-C 28$ & $121.6(2)$ \\
\hline 01 & - La & -06 & $71.50(5)$ & La & -01 & $-\mathrm{C} 1$ & $93.92(13)$ \\
\hline 01 & - La & -07 & $85.54(6)$ & La & -02 & $-C 1$ & $93.51(13)$ \\
\hline 01 & - La & -08 & $123.27(5)$ & $\mathrm{La}$ & -03 & $-C 11$ & $98.20(16)$ \\
\hline 01 & $-L a$ & $-C 21$ & $70.44(5)$ & La & -04 & $-C 11 \_a$ & $158.63(16)$ \\
\hline 01 & - La & -04_a & $128.09(6)$ & La_a & -04 & - C11_a & $90.70(14)$ \\
\hline 02 & - La & -03 & $73.04(6)$ & La & -04 & - La_a & $110.45(6)$ \\
\hline 02 & - La & -04 & $147.99(5)$ & La & -05 & $-C 2 \overline{1}$ & $95.91(14)$ \\
\hline 02 & - La & -05 & $119.79(5)$ & La & -06 & $-C 21$ & $90.53(14)$ \\
\hline
\end{tabular}


Table S6 (cont.) - Bond Angles (Degrees) for Compound 1b'

\begin{tabular}{|c|c|c|c|c|c|c|c|}
\hline 02 & $-\mathrm{La}$ & -06 & $117.93(5)$ & La & -07 & $-\mathrm{S} 1$ & $144.32(10)$ \\
\hline 02 & - La & -07 & $74.98(6)$ & La & -08 & $-\mathrm{S} 2 \mathrm{~A}$ & $124.70(10)$ \\
\hline 02 & $-\mathrm{La}$ & -08 & $73.14(5)$ & La & -08 & $-S 2 B$ & $156.88(15)$ \\
\hline 02 & $-\mathrm{La}$ & $-C 21$ & $120.44(5)$ & 01 & $-C 1$ & -02 & $122.4(2)$ \\
\hline 02 & $-\mathrm{La}$ & -04_a & $117.50(5)$ & 02 & $-\mathrm{C} 1$ & $-\mathrm{C} 2$ & $117.57(18)$ \\
\hline 03 & $-\mathrm{La}$ & -04 & $117.80(5)$ & 01 & $-C 1$ & $-\mathrm{C} 2$ & $120.02(19)$ \\
\hline 03 & - La & -05 & $75.37(5)$ & C1 & $-\mathrm{C} 2$ & $-\mathrm{C3}$ & $120.1(2)$ \\
\hline 03 & $-\mathrm{La}$ & -06 & $124.31(5)$ & C3 & $-\mathrm{C} 2$ & $-C 7$ & $120.6(2)$ \\
\hline 03 & $-\mathrm{La}$ & -07 & $145.65(5)$ & C1 & $-\mathrm{C} 2$ & $-C 7$ & $119.3(2)$ \\
\hline 03 & $-\mathrm{La}$ & -08 & $77.96(6)$ & $\mathrm{C} 2$ & $-\mathrm{C3}$ & $-\mathrm{C} 4$ & $118.4(3)$ \\
\hline 03 & $-\mathrm{La}$ & $-C 21$ & $99.31(6)$ & $\mathrm{C} 2$ & $-\mathrm{C3}$ & $-C 9$ & $121.3(2)$ \\
\hline 03 & - La & -04_a & $49.41(5)$ & $\mathrm{C} 4$ & $-\mathrm{C} 3$ & $-\mathrm{CO}$ & $120.3(3)$ \\
\hline 04 & $-\mathrm{La}$ & -05 & $92.19(5)$ & C3 & $-\mathrm{C} 4$ & $-\mathrm{C5}$ & $122.3(3)$ \\
\hline 04 & $-\mathrm{La}$ & -06 & $82.24(5)$ & C4 & $-\mathrm{C5}$ & $-\mathrm{C} 10$ & $122.1(3)$ \\
\hline 04 & $-\mathrm{La}$ & -07 & $84.39(6)$ & C6 & $-\mathrm{C5}$ & $-\mathrm{C} 10$ & $119.7(3)$ \\
\hline 04 & $-\mathrm{La}$ & -08 & $79.77(5)$ & C4 & $-\mathrm{C5}$ & $-\mathrm{c} 6$ & $118.2(2)$ \\
\hline 04 & $-\mathrm{La}$ & $-C 21$ & $88.80(5)$ & $\mathrm{C} 5$ & $-\mathrm{C} 6$ & $-C 7$ & $121.6(3)$ \\
\hline 04 & - La & $-04 \_a$ & $68.66(5)$ & $\mathrm{C} 2$ & $-C 7$ & $-\mathrm{C} 8$ & $121.1(2)$ \\
\hline 05 & $-\mathrm{La}$ & -06 & $51.09(6)$ & $\mathrm{C} 2$ & $-C 7$ & $-\mathrm{C} 6$ & $119.0(2)$ \\
\hline 05 & $-\mathrm{La}$ & -07 & $133.01(6)$ & C6 & $-C 7$ & $-\mathrm{C} 8$ & $119.9(2)$ \\
\hline 05 & $-\mathrm{La}$ & -08 & $144.36(6)$ & 03 & $-\mathrm{C} 11$ & $-\mathrm{C} 12$ & $118.7(2)$ \\
\hline 05 & $-\mathrm{La}$ & $-C 21$ & $25.52(7)$ & 03 & $-C 11$ & -04_a & $121.6(3)$ \\
\hline 04_a & $-\mathrm{La}$ & -05 & $71.65(5)$ & 04_a & $-C 11$ & $-\mathrm{C} 12$ & $119.7(2)$ \\
\hline 06 & $-\mathrm{La}$ & -07 & $82.11(6)$ & $\mathrm{C} 1 \overline{1}$ & $-\mathrm{C} 12$ & $-\mathrm{C} 17$ & $118.6(2)$ \\
\hline 06 & $-\mathrm{La}$ & -08 & $156.44(6)$ & C13 & $-\mathrm{C} 12$ & $-\mathrm{C} 17$ & $120.9(2)$ \\
\hline 06 & - La & $-C 21$ & $25.70(7)$ & C11 & $-C 12$ & $-C 13$ & $120.5(2)$ \\
\hline 04_a & $-\mathrm{La}$ & -06 & $113.97(5)$ & C14 & $-C 13$ & - C19 & $119.6(2)$ \\
\hline 07 & $-\mathrm{La}$ & -08 & $81.11(7)$ & C12 & $-C 13$ & $-\mathrm{C} 14$ & $118.1(2)$ \\
\hline 07 & $-\mathrm{La}$ & $-C 21$ & $107.50(7)$ & C12 & $-C 13$ & - C19 & $122.3(3)$ \\
\hline 04_a & $-\mathrm{La}$ & -07 & $145.27(5)$ & C13 & $-C 14$ & $-C 15$ & $122.3(2)$ \\
\hline 08 & $-\mathrm{La}$ & $-C 21$ & $165.06(6)$ & C14 & $-\mathrm{C} 15$ & $-C 20$ & $120.8(2)$ \\
\hline 04_a & $-\mathrm{La}$ & -08 & $73.10(6)$ & C16 & $-C 15$ & $-C 20$ & $121.0(3)$ \\
\hline 04_a & $-\mathrm{La}$ & $-C 21$ & $93.86(5)$ & C14 & $-C 15$ & - C16 & $118.2(3)$ \\
\hline 07 & $-\mathrm{S} 1$ & $-C 31$ & $104.67(14)$ & C15 & - C16 & $-\mathrm{C} 17$ & $121.8(2)$ \\
\hline 07 & $-\mathrm{S} 1$ & $-\mathrm{C} 32$ & $104.31(13)$ & C12 & $-\mathrm{C} 17$ & - C18 & $120.7(3)$ \\
\hline C31 & $-\mathrm{S1}$ & $-\mathrm{C} 32$ & $100.06(17)$ & C16 & $-\mathrm{C} 17$ & - C18 & $120.7(2)$ \\
\hline 08 & $-\mathrm{S} 2 \mathrm{~A}$ & $-\mathrm{C} 33$ & $101.61(15)$ & C12 & $-\mathrm{C} 17$ & $-\mathrm{C} 16$ & $118.6(2)$ \\
\hline 08 & $-S 2 A$ & - C34 & $103.1(2)$ & 05 & $-C 21$ & $-C 22$ & $118.1(2)$ \\
\hline C33 & $-S 2 A$ & $-\mathrm{C} 34$ & $95.1(2)$ & 06 & $-C 21$ & $-C 22$ & $120.0(2)$ \\
\hline C35 & $-\mathrm{S} 2 \mathrm{~B}$ & $-C 36$ & $93.1(4)$ & 05 & $-C 21$ & -06 & $121.85(19)$ \\
\hline 08 & $-S 2 B$ & $-C 35$ & $102.89(19)$ & $\mathrm{C} 21$ & $-C 22$ & $-C 27$ & $119.3(2)$ \\
\hline 08 & $-S 2 B$ & - C36 & $98.1(3)$ & $\mathrm{C} 23$ & $-\mathrm{C} 22$ & $-C 27$ & $120.5(2)$ \\
\hline C23 & $-C 24$ & $-C 25$ & $122.3(2)$ & $\mathrm{C} 21$ & $-\mathrm{C} 22$ & $-C 23$ & $120.2(2)$ \\
\hline $\mathrm{C} 24$ & $-C 25$ & $-C 26$ & $117.9(2)$ & $\mathrm{C} 24$ & $-C 23$ & $-C 29$ & $120.1(2)$ \\
\hline C24 & $-C 25$ & $-\mathrm{C} 30$ & $121.3(3)$ & $\mathrm{C} 22$ & $-\mathrm{C} 23$ & $-C 29$ & $121.4(2)$ \\
\hline C26 & $-C 25$ & $-\mathrm{C} 30$ & $120.9(3)$ & $\mathrm{C} 22$ & $-C 23$ & $-C 24$ & $118.5(2)$ \\
\hline C3 & $-\mathrm{C} 4$ & $-\mathrm{H} 41$ & $117.5(17)$ & $\mathrm{C} 23$ & $-C 29$ & $-H 291$ & $112.2(18)$ \\
\hline C5 & $-\mathrm{C} 4$ & $-\mathrm{H} 41$ & $120.3(17)$ & $\mathrm{C} 23$ & $-C 29$ & $-H 292$ & $109.6(18)$ \\
\hline C7 & $-\mathrm{C} 6$ & $-H 61$ & $120.7(17)$ & H291 & $-C 29$ & $-\mathrm{H} 292$ & $109(2)$ \\
\hline C5 & $-\mathrm{C} 6$ & $-\mathrm{H} 61$ & $117.6(16)$ & H291 & $-C 29$ & $-\mathrm{H} 293$ & $105(2)$ \\
\hline C7 & $-\mathrm{C} 8$ & $-H 81$ & $113(2)$ & $\mathrm{C} 23$ & $-C 29$ & $-H 293$ & $111.4(17)$ \\
\hline C7 & $-\mathrm{C} 8$ & $-H 83$ & $111(2)$ & H292 & $-C 29$ & $-H 293$ & $110(3)$ \\
\hline H81 & $-\mathrm{C} 8$ & $-\mathrm{H} 82$ & $106(2)$ & $\mathrm{C} 25$ & $-\mathrm{C} 30$ & - H302 & $113(4)$ \\
\hline H81 & $-\mathrm{C} 8$ & $-\mathrm{H} 83$ & $108(2)$ & $\mathrm{C} 25$ & $-\mathrm{C} 30$ & $-\mathrm{H} 303$ & $114(4)$ \\
\hline H82 & $-\mathrm{C} 8$ & $-\mathrm{H} 83$ & $109(3)$ & H301 & $-\mathrm{C} 30$ & $-\mathrm{H} 302$ & $103(5)$ \\
\hline C7 & $-\mathrm{C} 8$ & $-H 82$ & $110(2)$ & H301 & $-\mathrm{C} 30$ & $-\mathrm{H} 303$ & $106(5)$ \\
\hline C3 & $-C 9$ & -H91 & $115(2)$ & $\mathrm{H} 302$ & $-\mathrm{C} 30$ & $-\mathrm{H} 303$ & $109(5)$ \\
\hline C3 & $-C 9$ & $-\mathrm{H} 92$ & $112(2)$ & $\mathrm{C} 25$ & $-\mathrm{C} 30$ & - H301 & $111(3)$ \\
\hline H91 & $-\mathrm{CO}$ & $-\mathrm{H} 92$ & $107(3)$ & S1 & $-C 31$ & - H311 & $106(2)$ \\
\hline H91 & $-C 9$ & $-\mathrm{H} 93$ & $102(3)$ & $\mathrm{S} 1$ & - C31 & - H312 & $106.0(19)$ \\
\hline H92 & $-C 9$ & $-\mathrm{H} 93$ & $107(3)$ & H311 & $-C 31$ & $-H 312$ & $116(3)$ \\
\hline C3 & $-C 9$ & $-\mathrm{H} 93$ & $114(3)$ & H311 & - C31 & $-H 313$ & $110(3)$ \\
\hline C5 & $-\mathrm{C} 10$ & - H101 & $113.0(18)$ & H312 & - C31 & - H313 & $110(3)$ \\
\hline
\end{tabular}


Table S6 (cont.) - Bond Angles (Degrees) for Compound 1b'

\begin{tabular}{|c|c|c|c|c|c|c|c|}
\hline $\mathrm{C} 5$ & - C10 & $-\mathrm{H} 103$ & $112(2)$ & S1 & - C31 & $-H 313$ & $109(2)$ \\
\hline $\mathrm{H} 101$ & $-\mathrm{C} 10$ & $-\mathrm{H} 102$ & $109(3)$ & S1 & $-\mathrm{C} 32$ & $-H 322$ & $107.2(19)$ \\
\hline $\mathrm{C} 5$ & - C10 & $-\mathrm{H} 102$ & $112(2)$ & S1 & $-\mathrm{C} 32$ & - H323 & $108(2)$ \\
\hline $\mathrm{H} 102$ & - C10 & - H103 & $105(3)$ & S1 & $-\mathrm{C} 32$ & -H321 & $106(2)$ \\
\hline $\mathrm{H} 101$ & - C10 & - H103 & $105(3)$ & H321 & $-\mathrm{C} 32$ & $-H 323$ & $112(3)$ \\
\hline C13 & - C14 & $-\mathrm{H} 141$ & $117(2)$ & H322 & $-\mathrm{C} 32$ & - H323 & $113(3)$ \\
\hline C15 & - C14 & $-\mathrm{H} 141$ & $121(2)$ & H321 & - C32 & $-H 322$ & $111(3)$ \\
\hline C17 & - C16 & - H161 & $119.3(17)$ & & & & \\
\hline C15 & - C16 & - H161 & $118.9(17)$ & S2A & $-\mathrm{C} 33$ & -H331 & 109.48 \\
\hline C17 & - C18 & - H181 & $112.0(18)$ & S2A & - - 33 & -H332 & 109.52 \\
\hline C17 & - C18 & - H183 & $109(2)$ & H331 & $-\mathrm{C} 33$ & -H332 & 109.42 \\
\hline H181 & - C18 & $-\mathrm{H} 182$ & $111(3)$ & H331 & - C33 & $-H 333$ & 109.48 \\
\hline H181 & - C18 & - H183 & $106(3)$ & S2A & $-\mathrm{C} 33$ & - H333 & 109.46 \\
\hline H182 & - C18 & $-\mathrm{H} 183$ & $105(3)$ & H332 & - C33 & $-H 333$ & 109.47 \\
\hline $\mathrm{C} 17$ & - C18 & - H182 & $113(2)$ & S2A & - C34 & - H342 & 109.47 \\
\hline C13 & - C19 & - H191 & $113(2)$ & S2A & - - 34 & $-H 343$ & 109.42 \\
\hline C13 & - C19 & - H192 & $109(2)$ & S2A & - C34 & -H341 & 109.48 \\
\hline H191 & - C19 & - H192 & $112(3)$ & H341 & - C34 & - H343 & 109.51 \\
\hline H191 & - C19 & - H193 & $110(4)$ & H342 & - C34 & - H343 & 109.49 \\
\hline C13 & - C19 & - H193 & $112(3)$ & H341 & $-\mathrm{C} 34$ & - H342 & 109.46 \\
\hline H192 & - C19 & - H193 & $99(3)$ & S2B & $-C 35$ & -H351 & 109.51 \\
\hline C15 & $-\mathrm{C} 20$ & $-\mathrm{H} 2 \mathrm{O} 2$ & $113(4)$ & S2B & $-C 35$ & $-H 352$ & 109.52 \\
\hline C15 & $-\mathrm{C} 20$ & $-\mathrm{H} 203$ & $110(3)$ & S2B & $-\mathrm{C} 35$ & - H353 & 109.41 \\
\hline C15 & $-\mathrm{C} 20$ & $-H 201$ & $116(3)$ & H351 & $-C 35$ & - H352 & 109.47 \\
\hline H201 & $-\mathrm{C} 20$ & $-\mathrm{H} 203$ & $102(4)$ & H351 & $-\mathrm{C} 35$ & $-H 353$ & 109.53 \\
\hline $\mathrm{H} 2 \mathrm{O} 2$ & $-\mathrm{C} 20$ & $-\mathrm{H} 203$ & $107(4)$ & H352 & $-\mathrm{C} 35$ & -H353 & 109.39 \\
\hline H201 & $-\mathrm{C} 20$ & $-\mathrm{H} 202$ & $108(4)$ & S2B & - C36 & $-H 361$ & 109.40 \\
\hline $\mathrm{C} 25$ & $-\mathrm{C} 24$ & $-H 241$ & $119.7(16)$ & S2B & - C36 & $-H 362$ & 109.48 \\
\hline C23 & $-C 24$ & $-\mathrm{H} 241$ & $118.0(16)$ & S2B & - C36 & -H363 & 109.39 \\
\hline $\mathrm{C} 27$ & $-\mathrm{C} 26$ & $-H 261$ & $116.3(17)$ & H361 & - C36 & -H362 & 109.52 \\
\hline $\mathrm{C} 25$ & $-\mathrm{C} 26$ & $-H 261$ & $121.5(17)$ & H361 & - C36 & $-H 363$ & 109.49 \\
\hline $\mathrm{C} 27$ & $-\mathrm{C} 28$ & $-H 281$ & $113(2)$ & H362 & - C36 & -H363 & 109.55 \\
\hline $\mathrm{C} 27$ & $-C 28$ & $-\mathrm{H} 283$ & $111.4(18)$ & & & & \\
\hline H281 & $-C 28$ & $-H 282$ & $108(2)$ & & & & \\
\hline $\mathrm{C} 27$ & $-\mathrm{C} 28$ & $-\mathrm{H} 282$ & $110.2(17)$ & & & & \\
\hline H282 & $-\mathrm{C} 28$ & $-H 283$ & $109(3)$ & & & & \\
\hline H281 & $-C 28$ & $-H 283$ & $105(3)$ & & & & \\
\hline
\end{tabular}

Translation of Symmetry Code to Equiv.Pos

$a=[2555.00]=-x, y, 0.5-z$

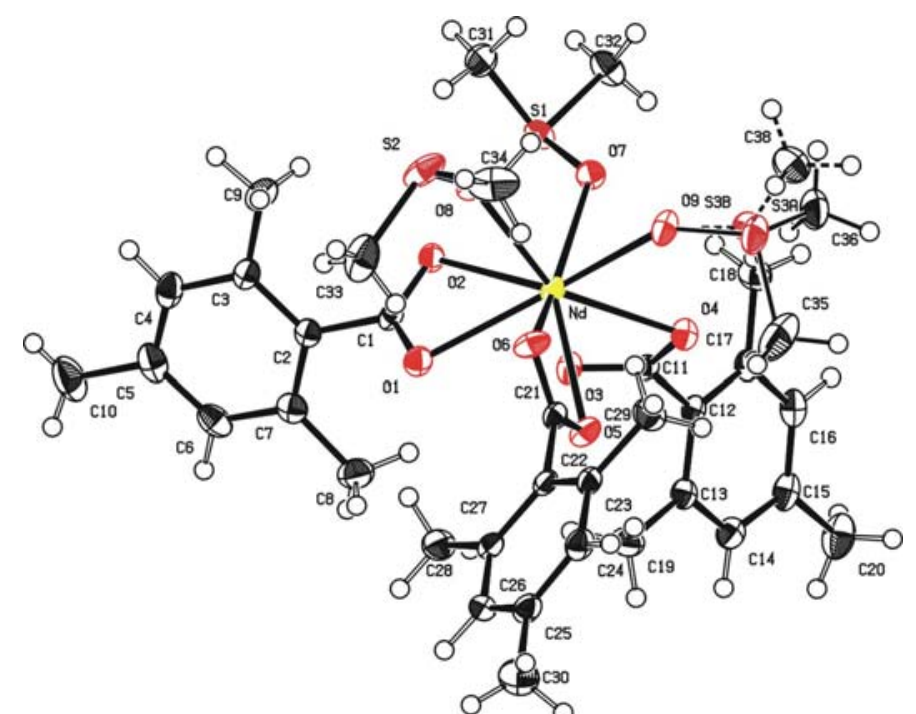

Molecular structure of $\left[\mathrm{Nd}\left(\mathrm{O}_{2} \mathrm{CC}_{6} \mathrm{H}_{2} \mathrm{Me}-2,4,6\right)_{3}(\mathrm{DMSO})_{3}\right]\left(\mathbf{1 c}^{\prime}\right)$ 
Table S7 - Crystal Data and Details of the Structure Determination for: Compound 1c'

Crystal Data

Formula

Formula Weight

Crystal System

Space group

$a, b, c$ [Angstrom]

alpha, beta, gamma [deg]

$\mathrm{V}[$ Ang**3]

Z

$D($ calc $)\left[\mathrm{g} / \mathrm{cm}^{*} * 3\right]$

$\operatorname{Mu}($ MoKa $)[/ \mathrm{mm}]$

$\mathrm{F}(000)$

Crystal Size $[\mathrm{mm}]$

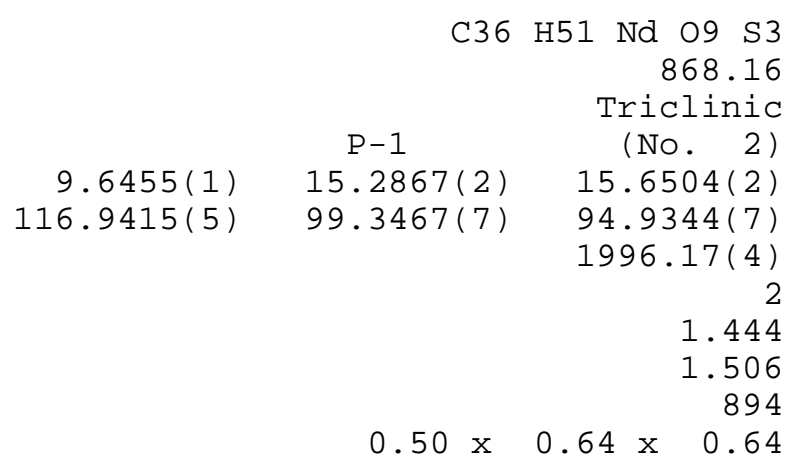

C36 H51 Nd 09 S3

868.16

Triclinic

$P-1$

(No. 2)

$9.6455(1) \quad 15.2867(2) \quad 15.6504(2)$

$116.9415(5)$

$99.3467(7)$

$94.9344(7)$

$1996.17(4)$

1.444

1.506

894

$0.50 \times 0.64 \times 0.64$

Data Collection

Temperature (K)

Radiation [Angstrom]

Theta Min-Max [Deg]

Dataset

Tot., Uniq. Data, R(int)

observed data $[\mathrm{I}>2.0 \mathrm{sigma}(\mathrm{I})]$

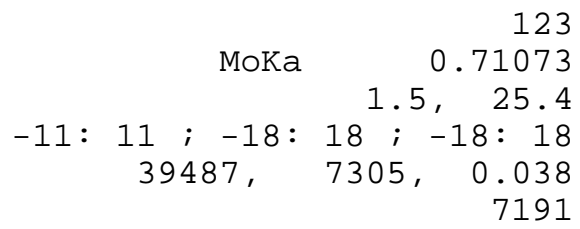

Refinement

Nref, Npar

$\mathrm{R}$, WR2, S

$7305, \quad 641$

$0.0196,0.0504,1.08$

$W=1 /\left[\backslash S^{\wedge} 2^{\wedge}\left(F 0^{\wedge} 2^{\wedge}\right)+(0.0203 P)^{\wedge} 2^{\wedge}+1.9522 P\right]$ where $P=\left(F 0^{\wedge} 2^{\wedge}+2 F c^{\wedge} 2^{\wedge}\right) / 3$

Max. and Av. Shift/Error

Min. and Max. Resd. Dens. [e/Ang^3]

$0.00,0.00$

$-0.81,1.44$

Table S8 - Final Coordinates and Equivalent Isotropic Displacement Parameters of the non-Hydrogen atoms for: Compound 1 $\mathbf{c}^{\prime}$

\begin{tabular}{|c|c|c|c|c|}
\hline Atom & $\mathrm{x}$ & $y$ & z & $\mathrm{U}(\mathrm{eq})$ [Ang \\
\hline & --- & - - & 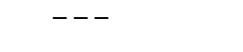 & \\
\hline $\mathrm{Nd}$ & $\odot .41282(1)$ & $0.12544(1)$ & $0.80516(1)$ & $0.0167(1)$ \\
\hline S1 & $0.31235(5)$ & $0.01865(4)$ & $0.95367(4)$ & $0.0226(1)$ \\
\hline S2 & $0.14690(7)$ & $0.30057(5)$ & $0.86993(5)$ & $0.0352(2)$ \\
\hline *S3A & $0.17548(9)$ & $-0.05765(7)$ & $0.55030(7)$ & $0.0326(3)$ \\
\hline 01 & $0.56681(16)$ & $0.29157(10)$ & ๑.92528(10) & $0.0248(4)$ \\
\hline 02 & $0.47817(15)$ & $0.20407(10)$ & $\odot .99062(10)$ & $0.0220(4)$ \\
\hline 03 & $0.63460(15)$ & ๑.06950(10) & ๑.85086(10) & $0.0228(4)$ \\
\hline 04 & $0.49607(14)$ & $-\odot .03580(10)$ & $0.70579(10)$ & $0.0217(4)$ \\
\hline 05 & $0.56922(15)$ & $\odot .16080(11)$ & $0.70365(10)$ & $0.0239(4)$ \\
\hline 06 & $0.38116(15)$ & $0.23410(11)$ & $0.72348(11)$ & $0.0261(5)$ \\
\hline 07 & $0.30109(15)$ & $\odot .00751(10)$ & $\odot .85096(10)$ & $0.0225(4)$ \\
\hline 08 & $0.22034(15)$ & $0.21589(10)$ & $\odot .86623(11)$ & $0.0241(4)$ \\
\hline 09 & ๑.21554(16) & $\odot .02500(11)$ & $\odot .65809(10)$ & $0.0277(4)$ \\
\hline C1 & $\odot .5554(2)$ & $\odot .27969(14)$ & $\odot .99887(14)$ & $0.0190(6)$ \\
\hline C2 & $0.6362(2)$ & $\odot .35795(14)$ & 1.09985(14) & $0.0192(6)$ \\
\hline C3 & $0.5609(2)$ & $\odot .40950(14)$ & $1.17158(15)$ & $0.0221(6)$ \\
\hline C4 & $0.6372(3)$ & $0.48193(16)$ & $1.26480(16)$ & $0.0286(7)$ \\
\hline C5 & $0.7855(3)$ & $\odot .50292(16)$ & $1.28886(16)$ & $0.0312(7)$ \\
\hline C6 & $0.8572(3)$ & $\odot .45076(16)$ & $1.21614(17)$ & ๑.0289(7) \\
\hline $\mathrm{C} 7$ & $0.7861(2)$ & $\odot .37913(15)$ & $1.12161(16)$ & $0.0236(6)$ \\
\hline C8 & $0.8698(3)$ & $0.3275(2)$ & $1.0451(2)$ & $0.0332(8)$ \\
\hline C9 & $0.3999(3)$ & $0.3895(2)$ & $1.14934(18)$ & $0.0301(7)$ \\
\hline C10 & $0.8663(4)$ & $0.5802(2)$ & $1.3907(2)$ & $\odot .0488(10)$ \\
\hline
\end{tabular}


Table s8 (cont.)

Atom
----
C11
C12
C13
C14
C15
C16
C17
C18
C19
C20
C21
C22
C23
C24
C25
C26
C27
C28
C29
C30
C31
C32
C33
C34
*C35
*C36
* $53 B$
*C38
*C37

- Final Coordinates and Equivalent Isotropic Displacement Parameters of the non-Hydrogen atoms for: Compound 1c'

\begin{tabular}{|c|c|c|}
\hline $\mathrm{y}$ & Z & $\mathrm{U}(\mathrm{eq}) \quad\left[\mathrm{Ang}^{\wedge}{ }^{\wedge}\right.$ \\
\hline-- & -- & \\
\hline$-0.01222(14)$ & $0.77232(14)$ & $0.0183(6)$ \\
\hline$-0.08305(14)$ & $0.75634(13)$ & $0.0185(6)$ \\
\hline$-0.05179(15)$ & $\odot .75135(14)$ & $0.0210(6)$ \\
\hline$-0.11874(16)$ & $0.73206(15)$ & $0.0248(6)$ \\
\hline$-0.21502(16)$ & $0.71717(15)$ & $0.0262(6)$ \\
\hline$-0.24340(16)$ & $0.72465(15)$ & $0.0253(6)$ \\
\hline$-0.17875(15)$ & $0.74417(14)$ & $0.0212(6)$ \\
\hline$-0.21108(19)$ & $0.75510(19)$ & $0.0303(7)$ \\
\hline ๑.05035(17) & $0.76246(19)$ & ๑. .0298(7) \\
\hline$-0.2875(2)$ & $0.6924(2)$ & 0.0392( 8$)$ \\
\hline $0.22167(14)$ & $0.69443(14)$ & $0.0196(6)$ \\
\hline $0.28279(14)$ & $0.64864(14)$ & $0.0187(5)$ \\
\hline $0.26988(15)$ & $0.55909(14)$ & $0.0209(6)$ \\
\hline $0.32670(16)$ & $0.51834(15)$ & $0.0252(6)$ \\
\hline ๑.39576(15) & $0.56429(16)$ & $0.0264(6)$ \\
\hline $0.40881(15)$ & $0.65436(16)$ & $0.0249(6)$ \\
\hline ๑. 35359(15) & $0.69789(15)$ & $0.0211(6)$ \\
\hline $0.3726(2)$ & $0.79792(17)$ & 0.0298(7) \\
\hline $0.19425(19)$ & $0.50316(18)$ & $0.0321(7)$ \\
\hline$\odot .4553(2)$ & $0.5176(2)$ & $0.0436(9)$ \\
\hline ๑. . $08474(19)$ & $0.99703(17)$ & $0.0311(7)$ \\
\hline$-0.10079(18)$ & $\odot .93450(18)$ & $0.0300(7)$ \\
\hline ๑. 40308(19) & $0.8998(2)$ & $0.0444(9)$ \\
\hline $0.2699(3)$ & $0.7450(2)$ & $0.0433(10)$ \\
\hline$-0.0544(3)$ & $0.5037(2)$ & ๑. . $0561(10)$ \\
\hline$-0.1724(2)$ & $0.5521(3)$ & $0.0434(11)$ \\
\hline$-0.07914(17)$ & $0.5922(2)$ & $0.0258(8)$ \\
\hline$-0.1072(8)$ & $\odot .5159(7)$ & $0.041(3)$ \\
\hline$-0.0544(3)$ & $0.5037(2)$ & 0.0561(10) \\
\hline
\end{tabular}

$U(e q)=1 / 3$ of the trace of the orthogonalized $U$ Tensor

Starred Atom sites have a S.O.F less than 1.0

Table S9 - Hydrogen Atom Positions and Isotropic Displacement Parameters for: Compound 10'

\begin{tabular}{|c|c|c|c|c|}
\hline Atom & $x$ & $\mathrm{y}$ & Z & $U($ iso $) \quad\left[A^{\prime} g^{\wedge} 2\right]$ \\
\hline--- & -- & -- & --- & $\ldots$ \\
\hline H41 & $0.585(3)$ & $\odot .5158(19)$ & $1.3120(19)$ & $\odot .033(7)$ \\
\hline H61 & $0.957(3)$ & $0.465(2)$ & $1.231(2)$ & ๑.041(7) \\
\hline H81 & $0.826(3)$ & $0.261(2)$ & 1. $001(2)$ & ๑.052(9) \\
\hline H82 & $0.878(4)$ & ๑.359(3) & $1.007(3)$ & $0.065(10)$ \\
\hline H83 & $0.963(4)$ & $\odot .327(3)$ & $1.072(3)$ & $0.075(11)$ \\
\hline H91 & $\odot .367(4)$ & $0.421(3)$ & $1.212(3)$ & $0.081(11)$ \\
\hline H92 & $0.362(4)$ & $0.419(3)$ & $1.116(3)$ & $0.085(12)$ \\
\hline H93 & $0.365(4)$ & $0.329(3)$ & $1.132(3)$ & $0.079(12)$ \\
\hline H101 & $0.817(5)$ & $0.613(3)$ & $1.430(3)$ & ๑. .098(16) \\
\hline $\mathrm{H} 102$ & $0.911(5)$ & $0.546(4)$ & $1.426(4)$ & $0.115(16)$ \\
\hline H103 & $0.941(5)$ & $\odot .615(3)$ & $1.394(3)$ & $0.091(14)$ \\
\hline H141 & $1.035(3)$ & $-0.0977(17)$ & $\odot .7299(17)$ & $\odot .025(6)$ \\
\hline H161 & $0.738(3)$ & $-0.309(2)$ & $0.7149(19)$ & $0.035(7)$ \\
\hline H181 & $0.499(3)$ & $-0.278(2)$ & $0.729(2)$ & $0.046(8)$ \\
\hline $\mathrm{H} 182$ & $0.516(4)$ & $-0.190(3)$ & $0.820(3)$ & $0.068(10)$ \\
\hline H183 & $0.456(3)$ & $-0.190(2)$ & $0.728(2)$ & 0.053(9) \\
\hline H191 & $0.983(3)$ & $0.054(2)$ & $0.745(2)$ & ๑. .044(8) \\
\hline H192 & $0.821(3)$ & ๑.067(2) & $0.723(2)$ & $0.047(8)$ \\
\hline H193 & $0.907(3)$ & $0.102(2)$ & $0.829(2)$ & ๑.038(7) \\
\hline
\end{tabular}


Table s9 (cont.) - Hydrogen Atom Positions and Isotropic Displacement Parameters for: Compound 10'

\begin{tabular}{|c|c|c|c|c|}
\hline Atom & $x$ & $y$ & z & $U($ iso $) \quad\left[\mathrm{Ang}^{\wedge} 2\right]$ \\
\hline--- & -- & -- & -- & --------- \\
\hline $\mathrm{H} 201$ & $1.012(4)$ & $-0.316(3)$ & $0.726(3)$ & $\odot .075(12)$ \\
\hline $\mathrm{H} 2 \mathrm{O} 2$ & $0.979(4)$ & $-0.336(3)$ & $0.626(3)$ & $0.072(11)$ \\
\hline $\mathrm{H} 203$ & $1.106(5)$ & $-0.255(3)$ & $0.710(3)$ & $0.093(13)$ \\
\hline $\mathrm{H} 241$ & $0.471(3)$ & $0.3160(17)$ & $0.4585(19)$ & $\odot .025(6)$ \\
\hline H261 & $0.808(3)$ & $0.4575(19)$ & $0.6901(18)$ & $0.031(6)$ \\
\hline H281 & $0.695(3)$ & $0.388(2)$ & $0.843(2)$ & $0.041(7)$ \\
\hline $\mathrm{H} 282$ & $0.790(3)$ & $0.317(2)$ & $0.795(2)$ & $0.038(7)$ \\
\hline $\mathrm{H} 283$ & $0.832(3)$ & $0.428(2)$ & $0.8260(19)$ & $0.034(7)$ \\
\hline H291 & $0.283(3)$ & $0.185(2)$ & $0.543(2)$ & ๑. $056(9)$ \\
\hline H292 & $0.267(3)$ & $0.215(2)$ & $0.465(2)$ & $0.047(8)$ \\
\hline H293 & $0.353(3)$ & $0.132(2)$ & $0.460(2)$ & $0.049(8)$ \\
\hline H301 & $0.764(5)$ & $0.513(4)$ & $0.562(4)$ & $0.111(16)$ \\
\hline $\mathrm{H} 302$ & $0.744(5)$ & $0.417(3)$ & $0.466(4)$ & $0.107(15)$ \\
\hline H303 & $0.635(5)$ & $0.469(4)$ & $0.482(4)$ & $0.115(17)$ \\
\hline H311 & $0.200(3)$ & ๑.151(2) & $1.006(2)$ & $0.046(8)$ \\
\hline H312 & $0.086(3)$ & $0.051(2)$ & $0.951(2)$ & $0.038(7)$ \\
\hline H313 & $0.169(3)$ & ๑. $0893(19)$ & $1.057(2)$ & $0.037(7)$ \\
\hline H321 & $0.293(3)$ & $-0.145(2)$ & $0.903(2)$ & $0.046(8)$ \\
\hline H322 & $0.236(3)$ & $-0.0928(18)$ & ○.999(2) & $0.031(6)$ \\
\hline H323 & $0.141(3)$ & $-0.123(2)$ & $0.892(2)$ & $0.039(7)$ \\
\hline H331 & $0.341(3)$ & $0.425(2)$ & $0.964(3)$ & $0.054(9)$ \\
\hline H332 & $0.340(3)$ & $0.382(2)$ & $0.853(2)$ & $0.039(7)$ \\
\hline H333 & $0.240(4)$ & $0.451(3)$ & $0.898(3)$ & $0.072(11)$ \\
\hline H341 & $0.003(3)$ & $0.216(2)$ & $0.720(2)$ & $0.051(9)$ \\
\hline H342 & $0.036(3)$ & $0.323(2)$ & $0.745(2)$ & $0.048(8)$ \\
\hline H343 & $0.145(3)$ & $0.253(2)$ & $0.709(2)$ & $0.042(7)$ \\
\hline${ }^{*}$ H361 & 0.1065 & -0.1964 & 0.5669 & 0.052 \\
\hline${ }^{*} \mathrm{H} 362$ & 0.2762 & -0.1615 & 0.6027 & 0.052 \\
\hline${ }^{*} \mathrm{H} 363$ & 0.2032 & -0.2222 & 0.4876 & 0.052 \\
\hline *H352 & 0.3185 & -0.1174 & 0.4438 & 0.067 \\
\hline *H351 & 0.3332 & $\odot .0009$ & 0.4881 & 0.067 \\
\hline${ }^{*} \mathrm{H} 353$ & 0.4113 & -0.0448 & 0.5528 & 0.067 \\
\hline${ }^{*} \mathrm{H} 371$ & 0.4111 & -0.0464 & 0.5521 & 0.067 \\
\hline *H372 & 0.3345 & 0.0019 & ๑. 4896 & 0.067 \\
\hline${ }^{*} \mathrm{H} 373$ & 0.3174 & -0.1168 & 0.4429 & 0.067 \\
\hline${ }^{*}$ H381 & -0.0344 & -0.1059 & 0.5537 & 0.049 \\
\hline *H382 & 0.0237 & -0.1736 & 0.4590 & 0.049 \\
\hline * H383 & 0.0286 & -0.0574 & 0.4931 & 0.049 \\
\hline
\end{tabular}

The Temperature Factor has the Form of $\operatorname{Exp}(-\mathrm{T})$ Where

$T=8 *\left(\mathrm{Pi}^{* *} 2\right){ }^{*} U^{*}(\operatorname{Sin}($ Theta $) /$ Lambda $) *{ }^{*} 2$ for Isotropic Atoms

Starred Atom sites have a S.0.F less than 1.0

Table S10 - (An)isotropic Displacement Parameters for: Compound 1c'

\begin{tabular}{|c|c|c|c|c|c|c|}
\hline Atom & $U(1,1)$ or & $U(2,2)$ & $U(3,3)$ & $U(2,3)$ & $U(1,3)$ & $U(1,2)$ \\
\hline--- & $---\cdot-$ & $\ldots$ & $\ldots \ldots$ & $\ldots$ & $\ldots \ldots$ & ----- \\
\hline $\mathrm{Nd}$ & $0.0216(1)$ & ๑. .0165(1) & ๑. . 0155(1) & $0.0088(1)$ & $0.0083(1)$ & $0.0063(1)$ \\
\hline S1 & $0.0242(2)$ & $0.0241(3)$ & 0.0198(2) & $0.0136(2)$ & $-\odot .0 \odot \odot 3(2)$ & $-0.0028(2)$ \\
\hline $\mathrm{S} 2$ & $0.0481(3)$ & 0.0379(3) & $0.0471(4)$ & $0.0321(3)$ & $0.0337(3)$ & 0.0294(3) \\
\hline S3A & $0.0319(4)$ & 0.0338(5) & 0.0188(5) & $0.0021(4)$ & ๑.0009(3) & $0.0105(3)$ \\
\hline 01 & $0.0344(8)$ & $0.0223(7)$ & $0.0206(7)$ & $0.0116(6)$ & ๑.0106(6) & $0.0032(6)$ \\
\hline 02 & $0.0301(8)$ & $0.0175(7)$ & ๑.0189(7) & ๑.0084(6) & ๑.0088(6) & ๑.0016(6) \\
\hline 03 & $0.0251(7)$ & 0.0225(7) & 0.0163(7) & 0.0056(6) & ๑.0032(6) & ๑.0068(6) \\
\hline 04 & $0.0223(7)$ & ๑. . $0218(7)$ & 0.0185(7) & $0.0082(6)$ & ๑.0010(6) & $0.0066(6)$ \\
\hline
\end{tabular}


Table S10 (cont.) - (An)isotropic Displacement Parameters for: Compound 1c'

\begin{tabular}{|c|c|c|c|c|c|c|}
\hline Atom & $\mathrm{U}(1,1)$ or & $U(2,2)$ & $U(3,3)$ & $U(2,3)$ & $U(1,3)$ & $U(1,2)$ \\
\hline & & & & & & \\
\hline 5 & 0301 ( 8 ) & ○253(7) & $0.0248(7)$ & $0.0153(6)$ & (6) & $0.0118(6)$ \\
\hline 06 & $0272(8)$ & ○346 ( 8 ) & ๑. .0312( 8$)$ & $0236(7)$ & $160(6)$ & $0128(6)$ \\
\hline 07 & $283(7)$ & $0.0226(7)$ & 0.0194(7) & $0.0125(6)$ & ๑.0063(6) & $0.0021(6)$ \\
\hline 08 & $0301(8)$ & $0.0223(7)$ & $0.0308(8)$ & $0.0174(6)$ & 0.0165 & 0.0124(6) \\
\hline 09 & $315(8)$ & $0.0275(8)$ & $0.0187(7)$ & $0.0054(6)$ & $0.0057(6)$ & $0.0111(6)$ \\
\hline C1 & $0241(10)$ & ๑.0171(9) & ๑. . $0188(10)$ & $\odot .0086(8)$ & $\odot .0 \odot 97(8)$ & $\odot .0087(8)$ \\
\hline $\mathrm{C} 2$ & $0271(10)$ & $0.0146(9)$ & $0.0202(10)$ & $0.0114(8)$ & $0.0068(8)$ & $0.0044(8)$ \\
\hline C3 & $0.0334(11)$ & $0.0172(9)$ & $0.0209(10)$ & $0.0117(8)$ & 0.0102(9) & $0.0067(8)$ \\
\hline 54 & $.0471(14)$ & $0.0197(10)$ & $0.0212(11)$ & $0.0101(9)$ & $0.0123(10)$ & $0.0065(10)$ \\
\hline 5 & $479(14)$ & $.0227(11)$ & $0.0213(11)$ & 0.01 & $\odot .00$ & -0.00 \\
\hline C6 & $295(12)$ & $0.0269(11)$ & $0.0325(12)$ & $0.0194(10)$ & $-0.0001(9)$ & $-0.0020(9)$ \\
\hline C7 & $290(11)$ & ๑. . $0198(10)$ & $0.0276(11)$ & 0.0159(9) & $0.0067(9)$ & 0.0046 ( 8) \\
\hline C8 & $289(13)$ & $0.0349(14)$ & $\odot .0400(14)$ & ๑. . $0190(12)$ & $0.0140(11)$ & $0.0082(10)$ \\
\hline C9 & $336(12)$ & ๑.0323(13) & ๑. .0281(12) & $0.0140(11)$ & $0.0147(10)$ & $0.0123(10)$ \\
\hline C10 & $.065(2)$ & $0.0405(16)$ & $0.0248(13)$ & 0.0106(12 & $-0.0019(13)$ & $-0.0180(15)$ \\
\hline 11 & $216(10)$ & 0.0197(10) & $0.0162(9)$ & $\odot .0 \odot 99(8)$ & 0.0065 & $\odot .0 \odot$ \\
\hline C12 & $(10)$ & $(10)$ & $0.0117(9)$ & $0.0071(8)$ & $0.0017(7)$ & 0.00 \\
\hline C13 & $22(10)$ & $226(10)$ & $159(9)$ & $0.0082(8)$ & $0007(8)$ & $0.0047(8)$ \\
\hline C14 & $0209(11)$ & $0.0304(11)$ & ๑. . $0190(10)$ & $\odot .009 \odot(9)$ & $\odot .0009(8)$ & $\odot .0069(9)$ \\
\hline C15 & $316(11)$ & $0.0270(11)$ & $0.0165(10)$ & $0.0078(9)$ & $-0.0001(8)$ & $0.0131(9)$ \\
\hline C16 & $0.0376(12)$ & $0.0193(10)$ & $0.0180(10)$ & $0.0093(8)$ & $0.0011(9)$ & 0.00 \\
\hline 17 & $66(10)$ & ๑. $0219(10)$ & $138(9)$ & $\odot .0085(8)$ & $\odot .001$ & $\odot$. \\
\hline C18 & $(13)$ & 12) & $24(13)$ & 0.01 & 0.008 & 10) \\
\hline C19 & $34(11)$ & $0.0274(12)$ & $0.0394(14)$ & $0.0159(11)$ & 0.009 & $0.0046(9)$ \\
\hline C20 & $428(16)$ & ๑. . $0358(14)$ & $0.0339(14)$ & ๑. . 0113(12) & $\odot .0035(12)$ & ๑. . $0228(12)$ \\
\hline C21 & $236(10)$ & $0.0203(10)$ & $0.0130(9)$ & $\odot .0061(8)$ & ๑. .0051( 8) & $0.0028(8)$ \\
\hline C22 & $0.0228(10)$ & $0.0185(9)$ & $0.0164(9)$ & $\odot .0075(8)$ & $0.0097(8)$ & $0.0064(8)$ \\
\hline 23 & $53(10)$ & $9(10)$ & 151(9) & $0.0068(8)$ & 0.006 & 0.0 \\
\hline C24 & $3(12)$ & $268(11)$ & $73(10)$ & 0.01 & ○. & 0.006 \\
\hline C25 & $7(12)$ & $0.0214(10)$ & $257(11)$ & 0.01 & ๑.0164(9) & $0.0055(9)$ \\
\hline C26 & $0246(11)$ & ๑. $0203(10)$ & $0.0257(11)$ & $0.0071(9)$ & ०.0096(9) & $-\odot . \odot \odot \odot 7(8)$ \\
\hline C27 & $.0205(10)$ & $0.0226(10)$ & ๑. .0194(10) & $0.0076(8)$ & $0.0086(8)$ & $0.0062(8)$ \\
\hline $\mathrm{C} 28$ & $.0233(11)$ & $0.0376(13)$ & ๑.0249(11) & $0.0134(10)$ & $0.0025(9)$ & $0.0022(10)$ \\
\hline C29 & $(13)$ & (13) & $0.0213(11)$ & 0.01 & 0.002 & -0.00 \\
\hline C30 & (18) & $\odot$. & $36(16)$ & $\odot .02$ & 0.02 & -0.00 \\
\hline C31 & $70(13)$ & $342(13)$ & $0.0216(11)$ & $0.0126(10)$ & ๑.0091(10) & $0.0030(10)$ \\
\hline C32 & $0.0364(13)$ & ๑. $0281(12)$ & $\odot .0273(12)$ & 0. 0194(10 & $-0.0015(10$ & $-0.0050(10)$ \\
\hline C33 & $0.079(2)$ & $\odot .0257(13)$ & $0.0403(15)$ & $0.0201(12)$ & $\odot .0247(15)$ & $0.0212(14)$ \\
\hline C34 & $0.0331(14)$ & $0.0600(18)$ & ๑.0645(19) & ๑. .0487(16) & ๑.0181(14) & ๑. . 0201(14) \\
\hline C35 & $0.0626(19)$ & $0.072(2)$ & $0.0292(14)$ & $0.0149(14$ & ๑. .0191(13) & 0.027 \\
\hline C36 & $0.059(2)$ & ๑. $0221(16)$ & ๑. . $0386(19)$ & $0.0 \odot 44(14)$ & ๑. .0182(17) & $0.0011(15)$ \\
\hline S3B & $0.0258(12)$ & $0.0293(13)$ & $0.0215(14)$ & $0.0129(10)$ & ๑.0019(10) & $0.0022(9)$ \\
\hline C38 & $0.023(5)$ & $0.050(6)$ & $0.033(5)$ & $0.011(5)$ & $-0.005(4)$ & $-0.002(4)$ \\
\hline C37 & ๑. .0626(19) & $\odot .072(2)$ & ๑. . $0292(14)$ & ๑. . $0149(14)$ & ๑. .0191(13) & ๑. $0273(16)$ \\
\hline
\end{tabular}

The Temperature Factor has the Form as given in Table S4

Table S11 - Bond Distances (Angstrom) for: Compound 1c'

$\begin{array}{llllll}\mathrm{Nd} & -01 & 2.5115(15) & \mathrm{C} 4 & -\mathrm{H} 41 & 0.94(3) \\ \mathrm{Nd} & -02 & 2.5260(14) & \mathrm{C} 6 & -\mathrm{H} 61 & 0.93(3) \\ \mathrm{Nd} & -03 & 2.4881(15) & \mathrm{C} 8 & -\mathrm{H} 81 & 0.94(3) \\ \mathrm{Nd} & -04 & 2.5321(15) & \mathrm{C} 8 & -\mathrm{H} 82 & 0.93(5) \\ \mathrm{Nd} & -05 & 2.5511(15) & \mathrm{C} 8 & -\mathrm{H} 83 & 0.93(4) \\ \mathrm{Nd} & -06 & 2.5241(18) & \mathrm{C} 9 & -\mathrm{H} 91 & 1.00(4) \\ \mathrm{Nd} & -07 & 2.4521(16) & \mathrm{C} 9 & -\mathrm{H} 92 & 0.89(5) \\ \mathrm{Nd} & -08 & 2.4683(15) & \mathrm{C} 9 & -\mathrm{H} 93 & 0.86(5) \\ \mathrm{Nd} & -09 & 2.4796(15) & \mathrm{C} 10 & -\mathrm{H} 101 & 0.84(5)\end{array}$


Table S11 (cont.) - Bond Distances (Angstrom) for: Compound 1c'

\begin{tabular}{|c|c|c|c|c|c|}
\hline $\mathrm{Nd}$ & $-\mathrm{C1}$ & $2.873(2)$ & $\mathrm{C} 10$ & $-\mathrm{H} 102$ & $0.99(6)$ \\
\hline $\mathrm{Nd}$ & $-\mathrm{C} 11$ & $2.866(2)$ & $\mathrm{C} 10$ & $-\mathrm{H} 103$ & $0.84(5)$ \\
\hline S1 & -07 & $1.5221(15)$ & C14 & $-\mathrm{H} 141$ & $0.92(3)$ \\
\hline S1 & $-C 31$ & $1.785(3)$ & C16 & $-H 161$ & $0.95(3)$ \\
\hline S1 & - C32 & $1.783(3)$ & C18 & - H181 & $0.91(3)$ \\
\hline S2 & -08 & $1.5113(18)$ & C18 & - H182 & $0.94(4)$ \\
\hline S2 & $-C 33$ & $1.794(4)$ & C18 & $-H 183$ & $0.89(3)$ \\
\hline S2 & - C34 & $1.788(3)$ & C19 & - H191 & $0.94(3)$ \\
\hline S3A & -09 & $1.5376(17)$ & C19 & - H192 & $0.99(3)$ \\
\hline S3A & $-C 35$ & $1.734(3)$ & C19 & -H193 & $0.96(3)$ \\
\hline S3A & - C36 & $1.788(4)$ & $\mathrm{C} 20$ & $-\mathrm{H} 201$ & $0.82(5)$ \\
\hline S3B & - C38 & $1.786(10)$ & $\mathrm{C} 20$ & $-\mathrm{H} 202$ & $0.94(4)$ \\
\hline S3B & -09 & $1.446(3)$ & $\mathrm{C} 20$ & $-\mathrm{H} 203$ & $0.96(5)$ \\
\hline S3B & $-\mathrm{C} 37$ & $2.042(4)$ & $\mathrm{C} 24$ & $-\mathrm{H} 241$ & $0.92(3)$ \\
\hline 01 & $-\mathrm{C1}$ & $1.264(3)$ & $\mathrm{C} 26$ & $-H 261$ & $0.96(3)$ \\
\hline 02 & $-C 1$ & $1.262(3)$ & $\mathrm{C} 28$ & $-H 281$ & $0.93(3)$ \\
\hline 03 & $-C 11$ & $1.260(3)$ & $\mathrm{C} 28$ & $-\mathrm{H} 282$ & $0.93(3)$ \\
\hline 04 & $-C 11$ & $1.262(2)$ & $\mathrm{C} 28$ & $-\mathrm{H} 283$ & $0.96(3)$ \\
\hline 05 & $-C 21$ & $1.259(3)$ & $\mathrm{C} 29$ & $-H 291$ & $0.90(3)$ \\
\hline 06 & $-C 21$ & $1.263(2)$ & $\mathrm{C} 29$ & $-\mathrm{H} 292$ & $0.97(3)$ \\
\hline C1 & $-\mathrm{C} 2$ & $1.504(3)$ & $\mathrm{C} 29$ & $-\mathrm{H} 293$ & $0.94(3)$ \\
\hline $\mathrm{C} 2$ & $-\mathrm{C3}$ & $1.404(3)$ & C30 & $-H 301$ & $0.91(6)$ \\
\hline C2 & $-C 7$ & $1.404(3)$ & C30 & $-\mathrm{H} 302$ & $0.94(5)$ \\
\hline C3 & $-\mathrm{Cg}$ & $1.507(4)$ & $\mathrm{C} 30$ & $-\mathrm{H} 303$ & $0.89(6)$ \\
\hline C3 & $-\mathrm{C4}$ & $1.394(3)$ & C31 & - H311 & $0.96(3)$ \\
\hline C4 & $-\mathrm{C5}$ & $1.388(4)$ & C31 & - H312 & $0.94(3)$ \\
\hline C5 & $-\mathrm{C6}$ & $1.392(4)$ & C31 & - H313 & $0.92(3)$ \\
\hline C5 & $-\mathrm{C} 10$ & $1.508(4)$ & C32 & $-H 321$ & $0.93(3)$ \\
\hline C6 & $-C 7$ & $1.390(3)$ & C32 & - H322 & $0.96(3)$ \\
\hline C7 & $-\mathrm{C} 8$ & $1.509(4)$ & C32 & $-H 323$ & $0.96(3)$ \\
\hline C11 & $-\mathrm{C} 12$ & $1.508(3)$ & C33 & $-H 331$ & $0.95(4)$ \\
\hline C12 & $-\mathrm{C} 13$ & $1.402(3)$ & C33 & - H332 & $0.93(3)$ \\
\hline C12 & $-\mathrm{C} 17$ & $1.400(3)$ & C33 & - H333 & $0.89(5)$ \\
\hline C13 & $-C_{14}$ & $1.393(3)$ & C34 & $-H 341$ & $0.91(3)$ \\
\hline C13 & - C19 & $1.507(4)$ & C34 & $-\mathrm{H} 342$ & $0.92(3)$ \\
\hline C14 & - C15 & $1.389(4)$ & C34 & $-H 343$ & $0.93(3)$ \\
\hline C15 & $-\mathrm{C} 20$ & $1.514(4)$ & & & \\
\hline C15 & - C16 & $1.391(3)$ & C35 & - H351 & 0.98 \\
\hline C16 & $-\mathrm{C} 17$ & $1.398(3)$ & C35 & - H352 & 0.98 \\
\hline C17 & - C18 & $1.506(4)$ & C35 & $-H 353$ & 0.98 \\
\hline $\mathrm{C} 21$ & $-C 22$ & $1.508(3)$ & C36 & - H361 & 0.98 \\
\hline $\mathrm{C} 22$ & $-C 23$ & $1.399(3)$ & C36 & $-H 362$ & 0.98 \\
\hline C22 & $-C 27$ & $1.401(3)$ & C36 & - H363 & 0.98 \\
\hline C23 & $-C 24$ & $1.395(3)$ & C37 & - H371 & 0.98 \\
\hline $\mathrm{C} 23$ & $-C 29$ & $1.509(4)$ & C37 & $-\mathrm{H} 372$ & 0.98 \\
\hline C24 & $-C 25$ & $1.384(3)$ & C37 & - H373 & 0.98 \\
\hline $\mathrm{C} 25$ & $-C 26$ & $1.390(3)$ & $\mathrm{C} 38$ & $-H 381$ & 0.98 \\
\hline C25 & $-C 30$ & $1.513(4)$ & C38 & - H382 & 0.98 \\
\hline C26 & $-C 27$ & $1.393(3)$ & C38 & $-H 383$ & 0.98 \\
\hline C27 & $-C 28$ & $1.511(3)$ & & & \\
\hline
\end{tabular}

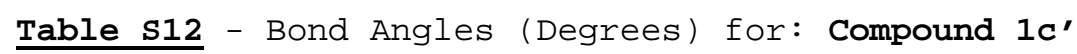

$\begin{array}{rrrrrrrr}01 & -\mathrm{Nd} & -02 & 52.09(5) & \mathrm{Nd} & -01 & -\mathrm{C} 1 & 93.12(13) \\ 01 & -\mathrm{Nd} & -03 & 80.12(5) & \mathrm{Nd} & -02 & -\mathrm{C} 1 & 92.50(11) \\ 01 & -\mathrm{Nd} & -04 & 127.06(5) & \mathrm{Nd} & -03 & -\mathrm{C} 11 & 94.00(12) \\ 01 & -\mathrm{Nd} & -05 & 76.29(5) & \mathrm{Nd} & -04 & -\mathrm{C} 11 & 91.90(12) \\ 01 & -\mathrm{Nd} & -06 & 74.69(5) & \mathrm{Nd} & -05 & -\mathrm{C} 21 & 92.51(12) \\ 01 & -\mathrm{Nd} & -07 & 124.62(5) & \mathrm{Nd} & -06 & -\mathrm{C} 21 & 93.65(14) \\ 01 & -\mathrm{Nd} & -08 & 82.23(5) & \mathrm{Nd} & -07 & -\mathrm{S} 1 & 127.79(9) \\ 01 & -\mathrm{Nd} & -09 & 149.19(6) & \mathrm{Nd} & -08 & -\mathrm{S} 2 & 143.07(10) \\ 01 & -\mathrm{Nd} & -\mathrm{C} 1 & 26.07(6) & \mathrm{Nd} & -09 & -\mathrm{S} \mathrm{A} & 145.53(10) \\ 01 & -\mathrm{Nd} & -\mathrm{C} 11 & 103.92(6) & \mathrm{Nd} & -09 & -\mathrm{S} 3 \mathrm{~B} & 122.20(15)\end{array}$


Table S12 (cont.) - Bond Angles (Degrees) for: Compound 1c'

\begin{tabular}{|c|c|c|c|c|c|c|c|}
\hline 02 & $-\mathrm{Nd}$ & -03 & $72.64(5)$ & $\mathrm{Nd}$ & $-\mathrm{C} 1$ & -01 & $60.81(11)$ \\
\hline 02 & $-\mathrm{Nd}$ & -04 & $118.49(5)$ & $\mathrm{Nd}$ & $-\mathrm{C1}$ & -02 & $61.46(10)$ \\
\hline 02 & $-\mathrm{Nd}$ & -05 & $123.85(5)$ & $\mathrm{Nd}$ & $-C 1$ & $-\mathrm{C2}$ & $177.22(14)$ \\
\hline 02 & $-\mathrm{Nd}$ & -06 & $119.90(5)$ & 01 & $-C 1$ & -02 & $122.19(18)$ \\
\hline 02 & $-\mathrm{Nd}$ & -07 & $72.54(5)$ & 01 & $-\mathrm{C} 1$ & $-\mathrm{C} 2$ & $119.2(2)$ \\
\hline 02 & $-\mathrm{Nd}$ & -08 & $73.02(5)$ & 02 & $-C 1$ & $-\mathrm{C} 2$ & $118.58(18)$ \\
\hline 02 & $-\mathrm{Nd}$ & -09 & $143.58(5)$ & C1 & $-\mathrm{C} 2$ & $-C 7$ & $119.86(18)$ \\
\hline 02 & $-\mathrm{Nd}$ & $-\mathrm{C} 1$ & $26.04(6)$ & C1 & $-\mathrm{C} 2$ & $-\mathrm{C3}$ & $119.64(17)$ \\
\hline 02 & $-\mathrm{Nd}$ & $-C 11$ & $95.57(5)$ & C3 & $-\mathrm{C} 2$ & $-C 7$ & $120.50(18)$ \\
\hline 03 & $-\mathrm{Nd}$ & -04 & $52.11(5)$ & $\mathrm{C} 2$ & $-\mathrm{C3}$ & $-\mathrm{C} 4$ & $119.0(2)$ \\
\hline 03 & $-\mathrm{Nd}$ & -05 & $78.86(5)$ & $\mathrm{C} 2$ & $-\mathrm{C3}$ & $-C 9$ & $121.46(19)$ \\
\hline 03 & $-\mathrm{Nd}$ & -06 & $128.04(5)$ & $\mathrm{C} 4$ & $-\mathrm{C3}$ & $-\mathrm{Cg}$ & $119.5(2)$ \\
\hline 03 & $-\mathrm{Nd}$ & -07 & $83.04(5)$ & C3 & $-\mathrm{C} 4$ & $-\mathrm{C5}$ & $121.7(2)$ \\
\hline 03 & $-\mathrm{Nd}$ & -08 & $145.40(5)$ & C6 & $-\mathrm{C5}$ & $-\mathrm{C} 10$ & $121.1(3)$ \\
\hline 03 & $-\mathrm{Nd}$ & -09 & $126.10(5)$ & $\mathrm{C} 4$ & $-\mathrm{C5}$ & $-\mathrm{C} 10$ & $120.9(2)$ \\
\hline 03 & $-\mathrm{Nd}$ & $-C 1$ & $74.10(5)$ & C4 & $-\mathrm{C5}$ & $-\mathrm{C} 6$ & $118.0(2)$ \\
\hline 03 & $-\mathrm{Nd}$ & $-C 11$ & $26.01(5)$ & $\mathrm{C5}$ & $-\mathrm{C} 6$ & $-C 7$ & $122.6(2)$ \\
\hline 04 & $-\mathrm{Nd}$ & -05 & $73.57(5)$ & $\mathrm{C} 2$ & $-C 7$ & $-\mathrm{C} 6$ & $118.2(2)$ \\
\hline 04 & $-\mathrm{Nd}$ & -06 & $114.97(5)$ & $\mathrm{C} 2$ & $-C 7$ & $-\mathrm{C} 8$ & $121.7(2)$ \\
\hline 04 & $-\mathrm{Nd}$ & -07 & $75.79(5)$ & C6 & $-C 7$ & $-\mathrm{C} 8$ & $120.0(2)$ \\
\hline 04 & $-\mathrm{Nd}$ & -08 & $150.25(5)$ & $\mathrm{Nd}$ & $-C 11$ & $-\mathrm{C} 12$ & $178.92(14)$ \\
\hline 04 & $-\mathrm{Nd}$ & -09 & $74.23(5)$ & 03 & $-C 11$ & $-\mathrm{C} 12$ & $119.03(17)$ \\
\hline 04 & $-\mathrm{Nd}$ & $-C 1$ & $126.19(5)$ & 04 & $-C 11$ & $-\mathrm{C} 12$ & $118.97(18)$ \\
\hline 04 & $-\mathrm{Nd}$ & $-\mathrm{C} 11$ & $26.10(5)$ & 03 & $-C 11$ & -04 & $122.0(2)$ \\
\hline 05 & $-\mathrm{Nd}$ & -06 & $51.49(5)$ & $\mathrm{Nd}$ & $-C 11$ & -03 & $59.99(11)$ \\
\hline 05 & $-\mathrm{Nd}$ & -07 & $149.35(5)$ & $\mathrm{Nd}$ & $-C 11$ & -04 & $62.0 \odot(11)$ \\
\hline 05 & $-\mathrm{Nd}$ & -08 & $125.06(6)$ & C13 & $-\mathrm{C} 12$ & $-\mathrm{C} 17$ & $121.0(2)$ \\
\hline 05 & $-\mathrm{Nd}$ & -09 & $92.04(5)$ & C11 & $-\mathrm{C} 12$ & $-\mathrm{C} 17$ & $120.41(18)$ \\
\hline 05 & $-\mathrm{Nd}$ & $-C_{1}$ & $99.98(5)$ & C11 & $-\mathrm{C} 12$ & $-\mathrm{C} 13$ & $118.5(2)$ \\
\hline 05 & $-\mathrm{Nd}$ & $-C 11$ & $74.84(6)$ & C14 & $-\mathrm{C} 13$ & - C19 & $119.91(19)$ \\
\hline 06 & $-\mathrm{Nd}$ & -07 & $147.96(5)$ & $\mathrm{C} 12$ & $-\mathrm{C} 13$ & $-\mathrm{C} 19$ & $121.43(19)$ \\
\hline 06 & $-\mathrm{Nd}$ & -08 & $74.29(5)$ & $\mathrm{C} 12$ & $-\mathrm{C} 13$ & $-C 14$ & $118.6(2)$ \\
\hline 06 & $-\mathrm{Nd}$ & -09 & $75.77(5)$ & C13 & - C14 & $-C 15$ & $121.7(2)$ \\
\hline 06 & $-\mathrm{Nd}$ & $-\mathrm{C} 1$ & $97.87(6)$ & C14 & $-C 15$ & - C16 & $118.5(2)$ \\
\hline 06 & $-\mathrm{Nd}$ & $-C 11$ & $125.51(5)$ & C14 & $-C 15$ & $-\mathrm{C} 20$ & $120.6(2)$ \\
\hline 07 & $-\mathrm{Nd}$ & -08 & $82.90(5)$ & C16 & $-C 15$ & $-C 20$ & $120.9(2)$ \\
\hline 07 & $-\mathrm{Nd}$ & -09 & $78.92(5)$ & C15 & $-\mathrm{C} 16$ & $-\mathrm{C} 17$ & $121.8(2)$ \\
\hline 07 & $-\mathrm{Nd}$ & $-C 1$ & $98.55(6)$ & C12 & $-\mathrm{C} 17$ & $-\mathrm{C} 18$ & $121.5(2)$ \\
\hline 07 & $-\mathrm{Nd}$ & $-C_{11}$ & $78.04(6)$ & C12 & $-\mathrm{C} 17$ & $-\mathrm{C} 16$ & $118.25(19)$ \\
\hline 08 & $-\mathrm{Nd}$ & -09 & $81.55(5)$ & C16 & $-\mathrm{C} 17$ & $-\mathrm{C} 18$ & $120.2(2)$ \\
\hline 08 & $-\mathrm{Nd}$ & $-C 1$ & $76.98(5)$ & 05 & $-C 21$ & -06 & $121.9(2)$ \\
\hline 08 & $-\mathrm{Nd}$ & $-C 11$ & $160.08(6)$ & 05 & $-C 21$ & $-\mathrm{C} 22$ & $119.45(18)$ \\
\hline 09 & $-\mathrm{Nd}$ & $-C 1$ & $158.53(5)$ & 06 & $-C 21$ & $-\mathrm{C} 22$ & $118.7(2)$ \\
\hline 09 & $-\mathrm{Nd}$ & $-C 11$ & $100.21(6)$ & $\mathrm{C} 23$ & $-\mathrm{C} 22$ & $-\mathrm{C} 27$ & $120.6(2)$ \\
\hline C1 & $-\mathrm{Nd}$ & $-C 11$ & $100.09(6)$ & $\mathrm{C} 21$ & $-\mathrm{C} 22$ & $-\mathrm{C} 27$ & $118.98(18)$ \\
\hline 07 & $-\mathrm{S} 1$ & $-C 31$ & $104.44(11)$ & C21 & $-C 22$ & $-C 23$ & $120.39(19)$ \\
\hline 07 & $-\mathrm{S} 1$ & $-\mathrm{C} 32$ & $105.10(11)$ & $\mathrm{C} 22$ & $-C 23$ & $-C 24$ & $118.66(19)$ \\
\hline C31 & $-\mathrm{S} 1$ & $-\mathrm{C} 32$ & $98.56(14)$ & $\mathrm{C} 22$ & $-C 23$ & $-C 29$ & $122.5(2)$ \\
\hline 08 & $-\mathrm{S} 2$ & $-\mathrm{C} 33$ & $106.89(14)$ & C24 & $-C 23$ & $-C 29$ & $118.80(19)$ \\
\hline 08 & $-\mathrm{S} 2$ & $-\mathrm{C} 34$ & $106.35(15)$ & $\mathrm{C} 23$ & $-\mathrm{C} 24$ & $-C 25$ & $122.0(2)$ \\
\hline C33 & $-\mathrm{S} 2$ & - C34 & $98.27(17)$ & $\mathrm{C} 26$ & $-C 25$ & $-\mathrm{C} 30$ & $121.1(2)$ \\
\hline 09 & $-S 3 A$ & - C35 & $106.08(13)$ & C24 & $-C 25$ & $-C 26$ & $118.1(2)$ \\
\hline 09 & -S3A & $-\mathrm{C} 36$ & $106.64(16)$ & $\mathrm{C} 24$ & $-C 25$ & $-\mathrm{C} 30$ & $120.8(2)$ \\
\hline C35 & $-S 3 A$ & - C36 & $94.1(2)$ & $\mathrm{C} 25$ & $-C 26$ & $-C 27$ & $122.1(2)$ \\
\hline C37 & $-S 3 B$ & - C38 & $97.7(4)$ & $\mathrm{C} 22$ & $-\mathrm{C} 27$ & $-C 26$ & $118.50(19)$ \\
\hline 09 & $-S 3 B$ & $-\mathrm{C} 37$ & $95.7(2)$ & $\mathrm{C} 22$ & $-C 27$ & $-C 28$ & $120.6(2)$ \\
\hline 09 & $-S 3 B$ & $-C 38$ & $98.7(4)$ & $\mathrm{C} 26$ & $-C 27$ & $-C 28$ & $120.8(2)$ \\
\hline C3 & $-\mathrm{C} 4$ & $-\mathrm{H} 41$ & $117.9(17)$ & H291 & $-C 29$ & $-\mathrm{H} 292$ & $104(3)$ \\
\hline $\mathrm{C} 5$ & $-\mathrm{C} 4$ & $-\mathrm{H} 41$ & $120.4(17)$ & H291 & $-C 29$ & $-\mathrm{H} 293$ & $108(3)$ \\
\hline C5 & $-\mathrm{C} 6$ & $-\mathrm{H} 61$ & $118.9(17)$ & H292 & $-C 29$ & $-H 293$ & $108(2)$ \\
\hline C7 & $-\mathrm{C} 6$ & $-\mathrm{H} 61$ & $118.5(17)$ & $\mathrm{C} 25$ & $-\mathrm{C} 30$ & $-H 301$ & $113(4)$ \\
\hline $\mathrm{C7}$ & $-\mathrm{C} 8$ & $-H 81$ & $112.4(18)$ & $\mathrm{C} 25$ & $-\mathrm{C} 30$ & $-\mathrm{H} 302$ & $112(3)$ \\
\hline $\mathrm{C7}$ & $-\mathrm{C} 8$ & $-\mathrm{H} 82$ & $111(3)$ & $\mathrm{C} 25$ & - C30 & $-\mathrm{H} 303$ & $115(4)$ \\
\hline
\end{tabular}


Table S12 (cont.)

\begin{tabular}{|c|c|c|c|}
\hline $\mathrm{C} 7$ & $-\mathrm{C} 8$ & $-\mathrm{H} 83$ & $113(2)$ \\
\hline H81 & $-\mathrm{C} 8$ & $-\mathrm{H} 82$ & $106(3)$ \\
\hline H81 & $-\mathrm{C} 8$ & $-\mathrm{H} 83$ & $107(4)$ \\
\hline H82 & $-\mathrm{C} 8$ & $-\mathrm{H} 83$ & $106(4)$ \\
\hline C3 & $-\mathrm{C9}$ & $-\mathrm{H} 91$ & $109(2)$ \\
\hline C3 & $-\mathrm{C9}$ & $-\mathrm{H} 92$ & $112(3)$ \\
\hline C3 & $-\mathrm{C} 9$ & $-\mathrm{H} 93$ & $113(3)$ \\
\hline H91 & $-\mathrm{CO}$ & $-\mathrm{H} 92$ & $103(4)$ \\
\hline H91 & $-\mathrm{C9}$ & $-\mathrm{H} 93$ & $97(4)$ \\
\hline H92 & $-\mathrm{C} 9$ & $-\mathrm{H} 93$ & $120(4)$ \\
\hline C5 & $-\mathrm{C} 10$ & $-\mathrm{H} 101$ & $117(3)$ \\
\hline C5 & $-\mathrm{C} 10$ & $-\mathrm{H} 102$ & $109(3)$ \\
\hline C5 & $-\mathrm{C} 10$ & $-\mathrm{H} 103$ & $116(3)$ \\
\hline H101 & $-\mathrm{C} 10$ & $-\mathrm{H} 102$ & $100(4)$ \\
\hline H101 & $-\mathrm{C} 10$ & $-\mathrm{H} 103$ & $113(5)$ \\
\hline $\mathrm{H} 102$ & $-\mathrm{C} 10$ & $-\mathrm{H} 103$ & $98(5)$ \\
\hline C13 & - C14 & $-\mathrm{H} 141$ & $118.4(18)$ \\
\hline C15 & - C14 & $-\mathrm{H} 141$ & $119.9(18)$ \\
\hline C15 & - C16 & $-\mathrm{H} 161$ & $120.4(18)$ \\
\hline C17 & - C16 & - H161 & $117.7(18)$ \\
\hline C17 & $-\mathrm{C} 18$ & $-\mathrm{H} 181$ & $113.8(19)$ \\
\hline C17 & $-\mathrm{C} 18$ & - H182 & $115(3)$ \\
\hline C17 & - C18 & - H183 & $112(2)$ \\
\hline H181 & $-\mathrm{C} 18$ & $-\mathrm{H} 182$ & $101(4)$ \\
\hline H181 & -C18 & $-\mathrm{H} 183$ & $108(3)$ \\
\hline H182 & - C18 & - H183 & $106(3)$ \\
\hline C13 & - C19 & - H191 & $110(2)$ \\
\hline C13 & - C19 & - H192 & $111.4(19)$ \\
\hline C13 & - C19 & -H193 & $112(2)$ \\
\hline H191 & - C19 & - H192 & $111(3)$ \\
\hline H191 & - C19 & - H193 & $107(3)$ \\
\hline H192 & - C19 & - H193 & $105(3)$ \\
\hline C15 & $-\mathrm{C} 20$ & $-\mathrm{H} 201$ & $112(3)$ \\
\hline C15 & $-\mathrm{C} 20$ & $-\mathrm{H} 202$ & $110(3)$ \\
\hline C15 & $-\mathrm{C} 20$ & $-\mathrm{H} 203$ & $113(3)$ \\
\hline H201 & $-\mathrm{C} 20$ & $-\mathrm{H} 202$ & $108(4)$ \\
\hline $\mathrm{H} 201$ & $-\mathrm{C} 20$ & $-\mathrm{H} 203$ & $101(4)$ \\
\hline $\mathrm{H} 2 \mathrm{O} 2$ & $-\mathrm{C} 20$ & $-\mathrm{H} 203$ & $113(4)$ \\
\hline C23 & $-C 24$ & $-\mathrm{H} 241$ & $118.1(18)$ \\
\hline $\mathrm{C} 25$ & $-C 24$ & $-\mathrm{H} 241$ & $119.9(19)$ \\
\hline $\mathrm{C} 25$ & $-C 26$ & $-H 261$ & $121.1(17)$ \\
\hline $\mathrm{C} 27$ & $-C 26$ & $-H 261$ & $116.8(17)$ \\
\hline $\mathrm{C} 27$ & $-C 28$ & $-\mathrm{H} 281$ & $112.2(18)$ \\
\hline $\mathrm{C} 27$ & $-C 28$ & $-\mathrm{H} 282$ & $111.8(17)$ \\
\hline $\mathrm{C} 27$ & $-C 28$ & $-\mathrm{H} 283$ & $111.5(17)$ \\
\hline H281 & $-C 28$ & $-\mathrm{H} 282$ & $106(3)$ \\
\hline H281 & $-C 28$ & $-\mathrm{H} 283$ & $107(2)$ \\
\hline H282 & $-C 28$ & $-\mathrm{H} 283$ & $108(3)$ \\
\hline $\mathrm{C} 23$ & $-C 29$ & $-H 291$ & $112.6(18)$ \\
\hline $\mathrm{C} 23$ & $-C 29$ & $-\mathrm{H} 292$ & $112.4(19)$ \\
\hline C23 & $-C 29$ & $-\mathrm{H} 293$ & $110.5(18)$ \\
\hline
\end{tabular}

(Degrees) for: Compound 1c'

\begin{tabular}{|c|c|c|c|}
\hline H301 & $-\mathrm{C} 30$ & $-\mathrm{H} 302$ & $110(4)$ \\
\hline H301 & $-\mathrm{C} 30$ & $-\mathrm{H} 303$ & $109(5)$ \\
\hline H302 & $-\mathrm{C} 30$ & $-\mathrm{H} 303$ & $96(5)$ \\
\hline S1 & - C31 & $-H 311$ & $106.8(18)$ \\
\hline S1 & - C31 & $-\mathrm{H} 312$ & $109.4(18)$ \\
\hline S1 & $-C 31$ & $-H 313$ & $109.0(19)$ \\
\hline H311 & $-C 31$ & $-H 312$ & $112(3)$ \\
\hline H311 & - C31 & $-H 313$ & $108(3)$ \\
\hline H312 & $-C 31$ & $-H 313$ & $111(3)$ \\
\hline S1 & $-\mathrm{C} 32$ & $-H 321$ & $107(2)$ \\
\hline S1 & $-\mathrm{C} 32$ & $-H 322$ & $105.3(18)$ \\
\hline S1 & $-\mathrm{C} 32$ & $-H 323$ & $111(2)$ \\
\hline H321 & $-\mathrm{C} 32$ & $-H 322$ & $112(3)$ \\
\hline H321 & $-\mathrm{C} 32$ & $-H 323$ & 108(3) \\
\hline H322 & $-\mathrm{C} 32$ & $-H 323$ & $114(3)$ \\
\hline S2 & - C33 & $-H 331$ & $109(2)$ \\
\hline S2 & - C33 & $-H 332$ & $109(2)$ \\
\hline S2 & - C33 & $-H 333$ & $106(3)$ \\
\hline H331 & - C33 & $-H 332$ & $111(3)$ \\
\hline H331 & - C33 & $-H 333$ & $110(3)$ \\
\hline H332 & $-\mathrm{C} 33$ & $-H 333$ & $111(3)$ \\
\hline S2 & $-\mathrm{C} 34$ & $-H 341$ & $107.0(19)$ \\
\hline S2 & $-\mathrm{C} 34$ & $-\mathrm{H} 342$ & $108.1(17)$ \\
\hline S2 & - C34 & $-H 343$ & $110.0(18)$ \\
\hline H341 & - C34 & $-\mathrm{H} 342$ & $108(3)$ \\
\hline H341 & - C34 & $-\mathrm{H} 343$ & 109(3) \\
\hline H342 & $-\mathrm{C} 34$ & $-\mathrm{H} 343$ & $114(3)$ \\
\hline S3A & $-C 35$ & $-H 351$ & 109.43 \\
\hline S3A & $-C 35$ & $-H 352$ & 109.49 \\
\hline S3A & $-C 35$ & $-H 353$ & 109.49 \\
\hline H351 & $-C 35$ & $-H 352$ & 109.40 \\
\hline H351 & $-C 35$ & - H353 & 109.53 \\
\hline H352 & $-C 35$ & $-H 353$ & 109.48 \\
\hline S3A & $-\mathrm{C} 36$ & $-H 361$ & 109.48 \\
\hline S3A & $-\mathrm{C} 36$ & $-H 362$ & 109.46 \\
\hline S3A & $-\mathrm{C} 36$ & $-H 363$ & 109.45 \\
\hline H361 & - C36 & $-H 362$ & 109.49 \\
\hline H361 & $-\mathrm{C} 36$ & $-H 363$ & 109.49 \\
\hline H362 & $-\mathrm{C} 36$ & $-H 363$ & 109.46 \\
\hline S3B & $-\mathrm{C} 37$ & $-H 371$ & 85.94 \\
\hline S3B & $-\mathrm{C} 37$ & $-\mathrm{H} 372$ & 129.46 \\
\hline S3B & $-\mathrm{C} 37$ & $-\mathrm{H} 373$ & 109.70 \\
\hline H371 & $-\mathrm{C} 37$ & $-\mathrm{H} 372$ & 109.46 \\
\hline H371 & $-\mathrm{C} 37$ & $-\mathrm{H} 373$ & 109.48 \\
\hline H372 & $-\mathrm{C} 37$ & $-\mathrm{H} 373$ & 109.42 \\
\hline S3B & $-C 38$ & $-H 381$ & 109.47 \\
\hline S3B & $-\mathrm{C} 38$ & $-H 382$ & 109.50 \\
\hline S3B & $-\mathrm{C} 38$ & $-H 383$ & 109.44 \\
\hline H381 & $-\mathrm{C} 38$ & $-H 382$ & 109.49 \\
\hline H381 & $-\mathrm{C} 38$ & $-H 383$ & 109.51 \\
\hline H382 & $-\mathrm{C} 38$ & $-H 383$ & 109.41 \\
\hline
\end{tabular}




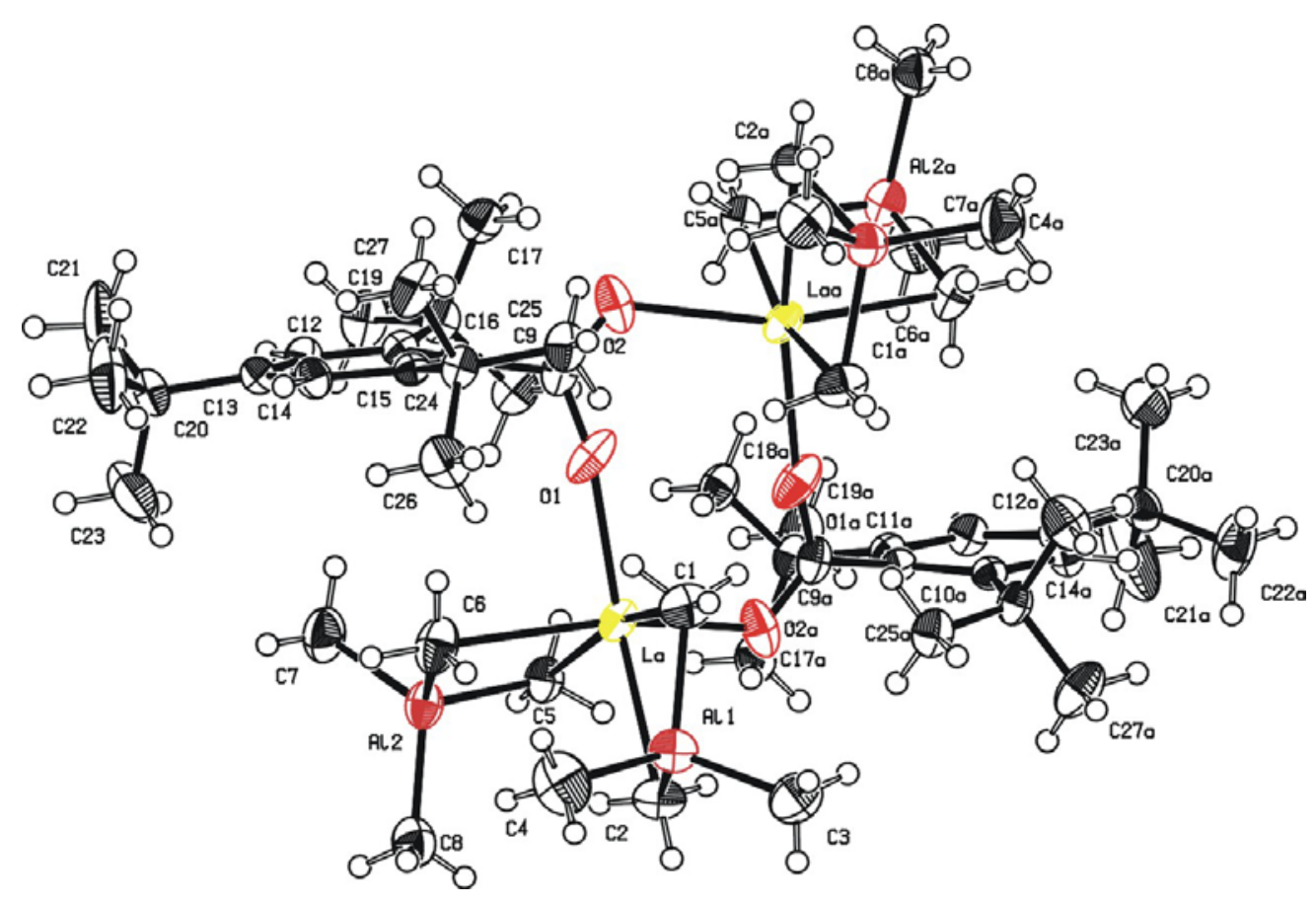

Molecular structure of $\left\{\mathrm{La}\left(\mathrm{O}_{2} \mathrm{CAr}{ }^{\mathrm{tBu}}\right)\left[(\mu-\mathrm{Me})_{2} \mathrm{AlMe}_{2}\right]_{2}\right\}_{2}$

$(9 b)$

Table S13 - Crystal Data and Details of the Structure Determination for: Compound $9 b$

\section{Crystal Data}

Formula

Formula Weight

Crystal System

space group

$a, b, c$ [Angstrom]

alpha, beta, gamma [deg]

$\mathrm{V}\left[\mathrm{Ang} *{ }^{*} 3\right]$

Z

$\mathrm{D}(\mathrm{calc})\left[\mathrm{g} / \mathrm{cm}^{*} * 3\right]$

$\mathrm{Mu}($ MoKa) $[/ \mathrm{mm}]$

$\mathrm{F}(0 \odot \odot)$

Crystal size [mm]
C54 H106 Al4 La2 04

1205.13

Monoclinic

C2/c (No. 15)

$23.7992(2) \quad 19.6717(1) \quad 15.1649(1)$

$90 \quad 114.6295(3)$

$6453.84(8)$

1.240

1.397

2512

$0.23 \times 0.30 \times 0.33$

\section{Data Collection}

Temperature (K)

Radiation [Angstrom]

Theta Min-Max [Deg]

Dataset

Tot., Uniq. Data, R(int)

Observed data [I $>2.0$ sigma(I)]

Refinement

Nref, Npar

$\mathrm{R}$, WR2, S

$W=1 /\left[\backslash \mathrm{s}^{\wedge} 2^{\wedge}\left(\mathrm{Fo}^{\wedge} 2^{\wedge}\right)+(0.0285 \mathrm{P})^{\wedge} 2^{\wedge}+7.6156 \mathrm{P}\right]$

Max. and $A v$. Shift/Error

Min. and Max. Resd. Dens. [e/Ang^3]
$5898, \quad 394$

$0.0244,0.0602,1.04$

MoKa $\quad 0.71073$

$1.7,25.3$

$-28: 28 ;-23: 23 ;-18: 18$ $72245, \quad 5898, \quad 0.046$

$\mathrm{re}=\left(\mathrm{FO}^{\wedge} 2^{\wedge}+2 \mathrm{FC} \mathrm{C}^{\wedge} \wedge\right) / 3$

$0.00,0.00$ $-0.87, \quad 0.70$ 
Table S14 - Final Coordinates and Equivalent Isotropic Displacement Parameters of the non-Hydrogen atoms for: Compound $\mathbf{9 b}$

\begin{tabular}{|c|c|c|c|c|}
\hline Atom & $\mathrm{x}$ & $\mathrm{y}$ & z & $\mathrm{U}(\mathrm{eq}) \quad\left[\mathrm{Ang}^{\wedge}{ }^{\wedge} 2\right]$ \\
\hline & $-\ldots$ & & & \\
\hline La & $-0.03208(1)$ & $0.24748(1)$ & $0.07588(1)$ & $0.0300(1)$ \\
\hline Al1 & $-0.07629(4)$ & $0.39433(4)$ & $-0.02925(6)$ & $0.0371(2)$ \\
\hline Al2 & $-0.00865(3)$ & $0.14968(4)$ & $-0.07867(6)$ & $0.0365(3)$ \\
\hline 01 & $0.05729(8)$ & $\odot .23371(9)$ & $0.22289(15)$ & $0.0470(6)$ \\
\hline 02 & $\odot .11569(9)$ & $\odot .21924(9)$ & $0.37785(13)$ & $0.0441(6)$ \\
\hline C1 & $-0.01556(14)$ & $0.38085(14)$ & $0.1145(2)$ & $0.0430(9)$ \\
\hline $\mathrm{C} 2$ & $-0.12184(14)$ & ๑.30366(15) & $-\odot .0803(2)$ & ๑.0419(9) \\
\hline C3 & $-0.13912(16)$ & $0.45846(17)$ & $-0.0282(3)$ & $0.0540(11)$ \\
\hline $\mathrm{C} 4$ & $-0.0282(2)$ & $0.4198(2)$ & $-0.1030(3)$ & $0.0667(16)$ \\
\hline C5 & $-0.06414(13)$ & $0.12456(13)$ & $-0.0085(2)$ & $\odot .0348(9)$ \\
\hline $\mathrm{C} 6$ & $0.04194(15)$ & $0.23611(16)$ & $-0.0177(3)$ & $0.0490(10)$ \\
\hline $\mathrm{C} 7$ & $0.05096(17)$ & $0.07427(17)$ & $-0.0492(3)$ & $0.0527(11)$ \\
\hline $\mathrm{c} 8$ & $-0.06454(15)$ & $0.16950(18)$ & $-0.2146(2)$ & $0.0475(11)$ \\
\hline c9 & $0.10941(11)$ & $0.21842(11)$ & $0.29189(18)$ & $0.0307(8)$ \\
\hline C10 & $0.16045(9)$ & $\odot .19742(11)$ & $0.26379(15)$ & $0.0241(6)$ \\
\hline $\mathrm{C} 11$ & $\odot .17072(9)$ & $0.12688(11)$ & $0.25424(16)$ & $0.0261(7)$ \\
\hline C12 & $0.20854(10)$ & $0.10997(11)$ & $0.20834(16)$ & $0.0311(7)$ \\
\hline C13 & $0.23780(10)$ & $0.15732(12)$ & $0.17406(16)$ & $0.0303(7)$ \\
\hline C14 & $0.23093(10)$ & $\odot .22476(12)$ & $0.19204(17)$ & $0.0307(7)$ \\
\hline C15 & ๑.19355(10) & $\odot .24700(10)$ & $0.23767(15)$ & $0.0245(6)$ \\
\hline $\mathrm{C} 16$ & $0.14519(10)$ & $0.06855(11)$ & $0.29613(17)$ & $0.0320(7)$ \\
\hline C17 & $0.17558(11)$ & $\odot .07279(13)$ & $0.40771(18)$ & $0.0396(8)$ \\
\hline $\mathrm{C} 18$ & $0.07429(11)$ & $\odot .06995(12)$ & $0.25746(19)$ & $0.0387(8)$ \\
\hline C19 & $0.16110(13)$ & $-0.00202(12)$ & $0.2693(2)$ & $0.0470(10)$ \\
\hline C20 & $0.27475(12)$ & $0.13314(13)$ & $\odot .11724(18)$ & $0.0379(8)$ \\
\hline $\mathrm{C} 21$ & $0.3115(2)$ & $0.0683(2)$ & $0.1632(3)$ & $0.106(2)$ \\
\hline $\mathrm{C} 22$ & $0.32176(15)$ & $0.18521(18)$ & $0.1172(3)$ & $0.0730(14)$ \\
\hline $\mathrm{C} 23$ & $0.23011(16)$ & $0.1177(2)$ & $0.0146(2)$ & $\odot .0827(16)$ \\
\hline C24 & ๑.19581(10) & $0.32492(11)$ & $0.25740(17)$ & $0.0299(7)$ \\
\hline C25 & $0.16140(11)$ & ๑.34981(12) & $0.31730(19)$ & $0.0388(8)$ \\
\hline C26 & $0.16949(14)$ & $0.36371(13)$ & $0.1611(2)$ & $0.0494(10)$ \\
\hline C27 & $\odot .26363(11)$ & ๑.34565(13) & $0.3155(2)$ & $0.0499(9)$ \\
\hline
\end{tabular}

$U(e q)=1 / 3$ of the trace of the orthogonalized $U$ Tensor

Table S15 - Hydrogen Atom Positions and Isotropic Displacement Parameters for: Compound $\mathbf{9 b}$

\begin{tabular}{|c|c|c|c|c|}
\hline Atom & $x$ & $y$ & z & $\mathrm{U}($ iso $) \quad[$ Ang^2] \\
\hline & -- & & -- & \\
\hline H11 & $\odot .0249(14)$ & $\odot .3622(15)$ & $\odot .133(2)$ & $\odot .061(9)$ \\
\hline $\mathrm{H} 12$ & $-0.0309(12)$ & $0.3639(15)$ & $0.164(2)$ & $0.057(8)$ \\
\hline H13 & $-0.0095(13)$ & $\odot .4289(17)$ & $0.126(2)$ & $\odot .068(9)$ \\
\hline H21 & $-0.1059(14)$ & $0.2681(16)$ & $-0.107(2)$ & $0.060(9)$ \\
\hline H22 & $-0.1507(14)$ & $\odot .3240(16)$ & $-0.133(2)$ & $0.066(10)$ \\
\hline H23 & $-0.1453(13)$ & $\odot .2824(15)$ & $-0.049(2)$ & $0.051(8)$ \\
\hline H31 & $-0.1579(16)$ & $0.4432(18)$ & $0.015(3)$ & $0.090(12)$ \\
\hline H32 & $-0.1695(16)$ & $0.4638(18)$ & $-0.089(3)$ & $0.090(13)$ \\
\hline H33 & $-0.1232(15)$ & $0.5015(19)$ & $-0.0 \odot 8(2)$ & $0.082(11)$ \\
\hline H41 & $-0.0208(18)$ & $0.383(2)$ & $-0.140(3)$ & $0.110(15)$ \\
\hline $\mathrm{H} 42$ & $\odot .0 \odot 9(2)$ & $0.438(2)$ & $-0.069(3)$ & $0.122(17)$ \\
\hline $\mathrm{H} 43$ & $-0.0470(18)$ & $0.456(2)$ & $-0.146(3)$ & $0.110(15)$ \\
\hline H51 & $-0.0472(12)$ & $0.1121(14)$ & $0.054(2)$ & $0.042(8)$ \\
\hline H52 & $-0.0744(11)$ & $0.0840(14)$ & $-0.0390(18)$ & $0.037(7)$ \\
\hline H53 & $-0.1030(12)$ & $0.1437(12)$ & $-0.0176(17)$ & $0.036(7)$ \\
\hline H61 & $0.0288(14)$ & $0.2801(18)$ & $-0.033(2)$ & $0.063(10)$ \\
\hline $\mathrm{H} 62$ & $0.0679(16)$ & $0.2331(15)$ & $-0.054(2)$ & $0.066(10)$ \\
\hline H63 & $0.0726(17)$ & $0.2340(16)$ & $0.044(3)$ & $0.073(11)$ \\
\hline
\end{tabular}




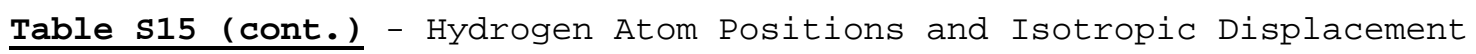
Parameters for: Compound $\mathbf{9 b}$

\begin{tabular}{|c|c|c|c|c|}
\hline Atom & $x$ & $\mathrm{y}$ & z & $\mathrm{U}($ iso $) \quad\left[\mathrm{Ang}^{\wedge} 2\right]$ \\
\hline---- & -- & --- & -- & --------- \\
\hline H71 & $\odot .0772(17)$ & ○.0690(19) & $0.020(3)$ & $0.097(13)$ \\
\hline H72 & $0.0765(15)$ & $0.0806(17)$ & $-0.080(2)$ & $0.078(11)$ \\
\hline H73 & ๑.0336(16) & $0.032(2)$ & $-0.067(3)$ & ○.096(14) \\
\hline H81 & $-0.1062(17)$ & $0.1717(16)$ & $-0.229(2)$ & $0.077(11)$ \\
\hline H82 & $-0.0601(14)$ & $0.1367(17)$ & $-0.258(2)$ & $0.074(10)$ \\
\hline H83 & $-0.0572(14)$ & $0.2096(19)$ & $-0.235(2)$ & $0.068(10)$ \\
\hline H121 & 0.2147 & 0.0632 & 0.1999 & 0.037 \\
\hline H141 & 0.2525 & $\odot .2579$ & 0.1726 & 0.037 \\
\hline H171 & 0.1681 & 0.1179 & 0.4282 & 0.059 \\
\hline H172 & 0.2202 & $\odot .0653$ & 0.4312 & 0.059 \\
\hline $\mathrm{H} 173$ & 0.1578 & 0.0379 & 0.4347 & 0.059 \\
\hline H181 & 0.0615 & 0.1111 & 0.2807 & 0.058 \\
\hline H182 & 0.0602 & $\odot .0297$ & 0.2807 & 0.058 \\
\hline H183 & 0.0560 & 0.0700 & 0.1864 & 0.058 \\
\hline H191 & 0.2061 & -0.0070 & 0.2951 & 0.071 \\
\hline H192 & 0.1431 & -0.0068 & ๑. 1985 & 0.071 \\
\hline H193 & 0.1442 & -0.0372 & 0.2971 & 0.071 \\
\hline H211 & ๑. 3335 & 0.0746 & 0.2335 & 0.160 \\
\hline H212 & 0.3413 & ๑. 0592 & 0.1355 & 0.160 \\
\hline H213 & $\odot .2830$ & ○. . 0298 & 0.1499 & 0.160 \\
\hline H221 & ๑ . 3510 & $\odot .1947$ & 0.1841 & 0.110 \\
\hline H222 & 0.3004 & 0.2272 & 0.0866 & 0.110 \\
\hline H223 & 0.3442 & 0.1673 & 0.0808 & 0.110 \\
\hline H231 & 0.2003 & 0.0835 & 0.0149 & 0.124 \\
\hline H232 & 0.2529 & 0.1002 & -0.0216 & 0.124 \\
\hline H233 & 0.2082 & 0.1593 & -0.0165 & 0.124 \\
\hline H251 & ๑.1761 & $\odot .3247$ & 0.3787 & ๑. . 058 \\
\hline H252 & 0.1169 & 0.3421 & 0.2808 & 0.058 \\
\hline $\mathrm{H} 253$ & $\odot .1692$ & ๑. 3985 & 0.3307 & 0.058 \\
\hline H261 & 0.1927 & ๑. 3516 & 0.1230 & 0.074 \\
\hline H262 & 0.1731 & 0.4127 & 0.1740 & 0.076 \\
\hline H263 & 0.1259 & 0.3517 & 0.1247 & 0.074 \\
\hline H271 & ๑. 2808 & 0.3204 & 0.3768 & $\odot .075$ \\
\hline H272 & ๑. 2659 & ๑. 3945 & 0.3290 & 0.075 \\
\hline $\mathrm{H} 273$ & 0.2874 & 0.3352 & 0.2778 & 0.075 \\
\hline
\end{tabular}

The Temperature Factor has the Form of $\operatorname{Exp}(-\mathrm{T})$ Where $T=8 *\left(\mathrm{Pi}^{* *} 2\right) \mathrm{U}^{*}(\operatorname{Sin}($ Theta $) /$ Lambda $){ }^{*} 2$ for Isotropic Atoms

Table S16 - (An)isotropic Displacement Parameters for: Compound 9b

\begin{tabular}{|c|c|c|c|c|c|c|}
\hline Atom & $\mathrm{U}(1,1)$ or & $U(2,2)$ & $U(3,3)$ & $U(2,3)$ & $U(1,3)$ & $U(1,2)$ \\
\hline- & ----- & & & -- & & - \\
\hline La & $0.0238(1)$ & $0.0219(1)$ & $0.0422(1)$ & $-0.0037(1)$ & ๑. $0118(1)$ & $-0.0013(1)$ \\
\hline Al1 & $0.0420(4)$ & $0.0274(4)$ & $0.0395(4)$ & $0.0026(3)$ & $0.0147(3)$ & $-0.0052(3)$ \\
\hline Al2 & $0.0361(4)$ & $0.0297(4)$ & $\odot .0480(5)$ & $-0.0053(3)$ & ๑.0219(4) & $-0.0004(3)$ \\
\hline 01 & $0.0234(9)$ & ०.0315(9) & $0.0793(14)$ & $0.0051(9)$ & $0.0148(9)$ & $0.0042(7)$ \\
\hline 02 & ๑. . $0691(12)$ & ○. $0298(9)$ & $0.0535(12)$ & $-0.0006(8)$ & $0.0456(10)$ & $-\odot .0 \odot \odot 4(9)$ \\
\hline C1 & $\odot .0462(17)$ & $0.0244(13)$ & $0.0473(16)$ & $-0.0023(12)$ & $0.0086(14)$ & $-0.0034(12)$ \\
\hline $\mathrm{C} 2$ & $0.0463(16)$ & $0.0346(15)$ & $\odot .0378(16)$ & $0.0038(13)$ & $0.0105(14)$ & $-0.0065(13)$ \\
\hline C3 & ๑. . $0553(19)$ & $\odot .0411(18)$ & $0.062(2)$ & ๑. .0०88(16) & ๑. $0208(18)$ & $\odot .0083(15)$ \\
\hline C4 & $0.077(3)$ & $0.059(2)$ & $0.077(3)$ & $0.004(2)$ & $0.045(2)$ & $-0.019(2)$ \\
\hline C5 & $0.0374(15)$ & $0.0263(13)$ & $\odot .0438(17)$ & $-0.0015(12)$ & $0.0201(13)$ & $0.0011(11)$ \\
\hline C6 & ๑. $0387(16)$ & ๑. $0418(18)$ & $0.073(2)$ & $-0.0110(16)$ & $\odot .0296(17)$ & $-0.0085(13)$ \\
\hline $\mathrm{C7}$ & ๑. . $0567(19)$ & $0.0450(18)$ & $0.064(2)$ & $-0.0004(16)$ & $0.0328(18)$ & $0.0133(15)$ \\
\hline
\end{tabular}




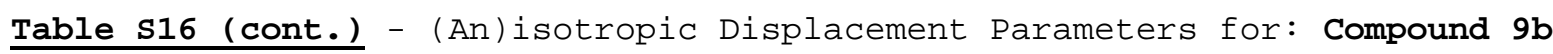

\begin{tabular}{|c|c|c|c|c|c|c|}
\hline Atom & $U(1,1)$ or & $U(2,2)$ & $U(3,3)$ & $U(2,3)$ & $U(1,3)$ & $U(1,2)$ \\
\hline 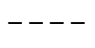 & -- & & & & & \\
\hline 8 & $466(18)$ & $0511(19)$ & $0509(18)$ & $-0.0037(15)$ & $263(15)$ & $-\odot$. \\
\hline $\mathrm{Cg}$ & $0.0340(13)$ & 0.0158(11) & $0.0481(15)$ & $0.0014(10)$ & $0.0228(12)$ & $-0.0016(9)$ \\
\hline 10 & ๑. $0224(11)$ & ๑. $0249(11)$ & ๑. . $0236(11)$ & $\odot . \odot \odot \odot \odot(9)$ & ๑. .0०81(9) & ๑. .0००3(9) \\
\hline 11 & $0.0244(11)$ & ๑. 0239(11) & ๑. $0284(12)$ & $-0.0002(9)$ & $0.0094(10)$ & $-0.0006(9)$ \\
\hline C12 & $0.0341(12)$ & ๑.0234(11) & $0.0368(13)$ & $-0.0035(10)$ & $0.0157(11)$ & $-0.0003(9)$ \\
\hline C13 & ๑. . $0304(12)$ & ๑. $0336(13)$ & ๑. $0269(12)$ & $-0.0032(10)$ & ๑.0119(10) & $0.0008(10)$ \\
\hline C14 & $0.0339(13)$ & ๑. 0296(12) & $0.0322(13)$ & $0.0004(10)$ & $0.0173(11)$ & $-0.0022(10)$ \\
\hline 15 & $0.0240(11)$ & ๑. $0232(11)$ & ๑. . $0241(11)$ & ๑. .0०18(9) & $0.0080(9)$ & $0.0014(9)$ \\
\hline 16 & $0.0326(13)$ & ๑. 0223(11) & $0.0434(14)$ & $0.0022(10)$ & $0.0181(11)$ & $-0.0007(9)$ \\
\hline 17 & $0.0404(14)$ & $0.0363(14)$ & $\odot .0439(15)$ & $0.0132(11)$ & ๑.0193(12) & $0.0045(11)$ \\
\hline C18 & $0.0368(14)$ & ๑.0286(13) & $\odot .0512(16)$ & $0.0035(11)$ & $0.0187(12)$ & $-0.0058(10)$ \\
\hline C19 & $0.0546(17)$ & ๑. $0244(13)$ & ๑.0711(19) & ๑.0018(12) & ๑.0352(15) & $-0.0023(11)$ \\
\hline $\mathrm{C} 20$ & $0.0460(15)$ & ๑.0390(14) & $0.0375(14)$ & $-0.0077(11)$ & ๑.0260(12) & $-0.0014(11)$ \\
\hline C21 & $0.158(4)$ & $0.089(3)$ & $0.138(4)$ & $0.043(3)$ & $0.127(4)$ & $0.071(3)$ \\
\hline C22 & $0.065(2)$ & $0.080(2)$ & $0.104(3)$ & $-0.045(2)$ & $0.065(2)$ & $-0.0270(18)$ \\
\hline $\mathrm{C} 23$ & $0.068(2)$ & $0.141(4)$ & $0.053(2)$ & $-0.041(2)$ & $\odot .039 \odot(18)$ & $-0.035(2)$ \\
\hline C24 & ๑.0295(12) & ๑. $0228(11)$ & $0.0404(13)$ & ๑. $00 \odot 1(10)$ & $0.0176(10)$ & $-0.0017(9)$ \\
\hline C25 & $0.0437(14)$ & ๑. $0261(12)$ & $0.0513(16)$ & $-0.0051(11)$ & ๑.0244(13) & $-0.0031(11)$ \\
\hline $\mathrm{C} 2$ & $0.070(2)$ & ๑. $0317(14)$ & $0.0530(17)$ & ๑.0086(13) & $\odot .0320(15)$ & $0.0055(13)$ \\
\hline $\mathrm{C} 27$ & $0.0371(14)$ & ๑.0322(14) & $0.080(2)$ & $-0.0130(14)$ & $0.0239(14)$ & $-0.0049(11)$ \\
\hline
\end{tabular}

The Temperature Factor has the Form as given in Table S4

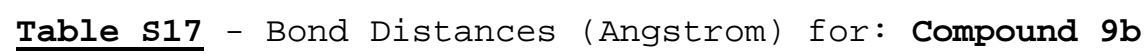

\begin{tabular}{|c|c|c|c|c|c|}
\hline La & -01 & $2.370(2)$ & $\mathrm{C} 4$ & $-\mathrm{H} 41$ & $0.98(4)$ \\
\hline La & $-C_{1}$ & $2.681(3)$ & $\mathrm{C} 4$ & $-\mathrm{H} 42$ & $0.89(5)$ \\
\hline La & $-\mathrm{C} 2$ & $2.679(3)$ & C4 & $-\mathrm{H} 43$ & $0.94(4)$ \\
\hline La & $-\mathrm{C5}$ & $2.693(3)$ & $\mathrm{C} 5$ & $-H 51$ & $0.90(3)$ \\
\hline La & $-\mathrm{C} 6$ & $2.693(4)$ & $\mathrm{C} 5$ & $-H 52$ & $0.90(3)$ \\
\hline La & $-02 \_a$ & $2.434(2)$ & $\mathrm{C} 5$ & $-H 53$ & $0.95(3)$ \\
\hline Al1 & $-\mathrm{C1}$ & $2.070(3)$ & $\mathrm{C} 6$ & $-H 61$ & $0.92(4)$ \\
\hline Al1 & $-\mathrm{C} 2$ & $2.063(3)$ & C6 & $-\mathrm{H} 62$ & $0.99(4)$ \\
\hline Al1 & $-\mathrm{C3}$ & $1.962(4)$ & $\mathrm{C} 6$ & $-\mathrm{H} 63$ & $0.92(4)$ \\
\hline Al1 & $-\mathrm{C4}$ & $1.969(5)$ & C7 & $-\mathrm{H} 71$ & $0.98(4)$ \\
\hline Al2 & $-\mathrm{C5}$ & $2.072(3)$ & C7 & $-\mathrm{H} 72$ & $0.92(4)$ \\
\hline Al2 & $-\mathrm{C} 6$ & $2.066(4)$ & C7 & $-\mathrm{H} 73$ & $0.92(4)$ \\
\hline Al2 & $-C 7$ & $1.971(4)$ & $\mathrm{C8}$ & $-H 81$ & $0.92(4)$ \\
\hline Al2 & $-\mathrm{C} 8$ & $1.971(3)$ & $\mathrm{C} 8$ & $-H 82$ & $0.96(3)$ \\
\hline 01 & $-\mathrm{CO}$ & $1.282(3)$ & $\mathrm{C} 8$ & $-\mathrm{H} 83$ & $0.89(4)$ \\
\hline 02 & $-C 9$ & $1.249(3)$ & & & \\
\hline C9 & $-\mathrm{C} 10$ & $1.503(4)$ & C12 & $-H 121$ & 0.95 \\
\hline C10 & $-\mathrm{C} 11$ & $1.427(3)$ & C14 & $-\mathrm{H} 141$ & 0.95 \\
\hline C10 & - C15 & $1.410(3)$ & C17 & - H171 & 0.98 \\
\hline C11 & $-\mathrm{C} 12$ & $1.388(3)$ & C17 & $-\mathrm{H} 172$ & 0.98 \\
\hline C11 & $-\mathrm{C} 16$ & $1.553(3)$ & C17 & $-\mathrm{H} 173$ & 0.98 \\
\hline C12 & $-\mathrm{C} 13$ & $1.387(3)$ & C18 & - H181 & 0.98 \\
\hline C13 & $-C 14$ & $1.378(3)$ & C18 & - H182 & 0.98 \\
\hline C13 & $-C 20$ & $1.541(4)$ & C18 & - H183 & 0.98 \\
\hline C14 & $-\mathrm{C} 15$ & $1.405(4)$ & C19 & $-H 191$ & 0.98 \\
\hline C15 & $-C 24$ & $1.558(3)$ & C19 & - H192 & 0.98 \\
\hline C16 & $-\mathrm{C} 17$ & $1.541(3)$ & C19 & - H193 & 0.98 \\
\hline C16 & - C18 & $1.539(4)$ & $\mathrm{C} 21$ & $-H 211$ & 0.98 \\
\hline C16 & - C19 & $1.538(3)$ & C21 & $-\mathrm{H} 212$ & 0.98 \\
\hline C2O & $-C 21$ & $1.539(5)$ & C21 & $-\mathrm{H} 213$ & 0.98 \\
\hline $\mathrm{C} 20$ & $-C 22$ & $1.517(5)$ & $\mathrm{C} 22$ & $-H 221$ & 0.98 \\
\hline C20 & $-C 23$ & $1.505(4)$ & $\mathrm{C} 22$ & $-\mathrm{H} 222$ & 0.98 \\
\hline C24 & $-C 25$ & $1.535(4)$ & $\mathrm{C} 22$ & $-\mathrm{H} 223$ & 0.98 \\
\hline
\end{tabular}


Table S17 (cont.) - Bond Distances (Angstrom) for: Compound 9b

$\begin{array}{llllll}\mathrm{C} 24 & -\mathrm{C} 26 & 1.531(4) & \mathrm{C} 23 & -\mathrm{H} 231 & 0.98 \\ \mathrm{C} 24 & -\mathrm{C} 27 & 1.537(4) & \mathrm{C} 23 & -\mathrm{H} 232 & 0.98 \\ & & & \mathrm{C} 23 & -\mathrm{H} 233 & 0.98 \\ \mathrm{C} 1 & -\mathrm{H} 11 & 0.96(3) & \mathrm{C} 25 & -\mathrm{H} 251 & 0.98 \\ \mathrm{C} 1 & -\mathrm{H} 12 & 1.02(3) & \mathrm{C} 25 & -\mathrm{H} 252 & 0.98 \\ \mathrm{C} 1 & -\mathrm{H} 13 & 0.96(3) & \mathrm{C} 25 & -\mathrm{H} 253 & 0.98 \\ \mathrm{C} 2 & -\mathrm{H} 21 & 0.96(3) & \mathrm{C} 26 & -\mathrm{H} 261 & 0.98 \\ \mathrm{C} 2 & -\mathrm{H} 22 & 0.90(3) & \mathrm{C} 26 & -\mathrm{H} 262 & 0.98 \\ \mathrm{C} 2 & -\mathrm{H} 23 & 0.97(3) & \mathrm{C} 26 & -\mathrm{H} 263 & 0.98 \\ \mathrm{C} 3 & -\mathrm{H} 31 & 0.98(4) & \mathrm{C} 27 & -\mathrm{H} 271 & 0.98 \\ \mathrm{C} 3 & -\mathrm{H} 32 & 0.91(4) & \mathrm{C} 27 & -\mathrm{H} 272 & 0.98 \\ \mathrm{C} 3 & -\mathrm{H} 33 & 0.93(4) & \mathrm{C} 27 & -\mathrm{H} 273 & 0.98\end{array}$

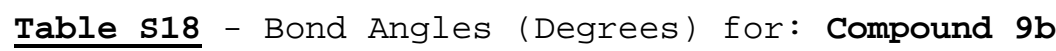

\begin{tabular}{|c|c|c|c|c|c|c|c|}
\hline 01 & $-\mathrm{La}$ & $-C 1$ & $85.06(8)$ & $\mathrm{C} 21$ & $-\mathrm{C} 20$ & $-C 22$ & $106.2(3)$ \\
\hline 01 & $-\mathrm{La}$ & $-\mathrm{C} 2$ & $162.04(8)$ & $\mathrm{C} 21$ & $-\mathrm{C} 20$ & $-C 23$ & $108.9(3)$ \\
\hline 01 & $-\mathrm{La}$ & $-\mathrm{C5}$ & $108.05(8)$ & $\mathrm{C} 22$ & $-\mathrm{C} 20$ & $-C 23$ & $109.8(3)$ \\
\hline 01 & $-\mathrm{La}$ & $-\mathrm{C} 6$ & $87.62(10)$ & C15 & $-\mathrm{C} 24$ & $-C 25$ & $116.3(2)$ \\
\hline 01 & $-\mathrm{La}$ & $-02 \_a$ & $102.93(7)$ & C15 & $-\mathrm{C} 24$ & $-C 26$ & $109.84(19)$ \\
\hline C1 & $-\mathrm{La}$ & $-\mathrm{C} 2$ & $77.28(9)$ & C15 & $-C 24$ & $-C 27$ & $108.4(2)$ \\
\hline $\mathrm{C} 1$ & $-\mathrm{La}$ & $-\mathrm{C5}$ & $165.72(8)$ & $\mathrm{C} 25$ & $-C 24$ & $-C 26$ & $107.4(2)$ \\
\hline C1 & $-\mathrm{La}$ & $-\mathrm{C6}$ & $97.34(10)$ & $\mathrm{C} 25$ & $-C 24$ & $-C 27$ & $105.5(2)$ \\
\hline 02_a & - La & $-\mathrm{C1}$ & $102.91(9)$ & C26 & $-C 24$ & $-C 27$ & $109.2(2)$ \\
\hline $\mathrm{C} 2$ & $-\mathrm{La}$ & $-\mathrm{C5}$ & $89.24(9)$ & & & & \\
\hline $\mathrm{C} 2$ & $-\mathrm{La}$ & $-\mathrm{C} 6$ & $91.38(11)$ & La & $-C 1$ & $-\mathrm{H} 11$ & $74.2(18)$ \\
\hline 02_a & - La & $-\mathrm{C} 2$ & $84.41(9)$ & La & $-C 1$ & $-\mathrm{H} 12$ & $77.0(17)$ \\
\hline $\mathrm{C} 5$ & $-\mathrm{La}$ & $-\mathrm{C} 6$ & $78.06(10)$ & $\mathrm{La}$ & $-C 1$ & $-\mathrm{H} 13$ & $177.8(18)$ \\
\hline 02_a & $-L a$ & $-C 5$ & $80.11(8)$ & Al1 & $-C 1$ & $-\mathrm{H} 11$ & $122.3(17)$ \\
\hline 02_a & $-\mathrm{La}$ & $-\mathrm{C} 6$ & $157.81(9)$ & Al1 & $-\mathrm{C} 1$ & $-\mathrm{H} 12$ & $120.7(17)$ \\
\hline $\mathrm{C} 1$ & -Al1 & $-\mathrm{C} 2$ & $108.15(12)$ & Al1 & $-C 1$ & $-\mathrm{H} 13$ & $92.9(17)$ \\
\hline C1 & -Al1 & $-\mathrm{C3}$ & $105.90(15)$ & $\mathrm{H} 11$ & $-C 1$ & $-\mathrm{H} 12$ & $107(2)$ \\
\hline C1 & -Al1 & $-\mathrm{C} 4$ & $108.34(17)$ & $\mathrm{H} 11$ & $-C 1$ & $-\mathrm{H} 13$ & $106(3)$ \\
\hline $\mathrm{C} 2$ & $-A 11$ & $-\mathrm{C3}$ & $106.02(15)$ & $\mathrm{H} 12$ & $-C 1$ & $-\mathrm{H} 13$ & $105(2)$ \\
\hline $\mathrm{C} 2$ & -Al1 & $-\mathrm{C4}$ & $110.22(15)$ & $\mathrm{La}$ & $-\mathrm{C} 2$ & $-\mathrm{H} 21$ & $76.3(17)$ \\
\hline C3 & -Al1 & $-\mathrm{C} 4$ & $117.80(17)$ & La & $-\mathrm{C} 2$ & $-\mathrm{H} 22$ & $177(2)$ \\
\hline C5 & $-A 12$ & $-\mathrm{C} 6$ & $110.11(14)$ & La & $-\mathrm{C} 2$ & $-\mathrm{H} 23$ & $78.4(18)$ \\
\hline C5 & $-A 12$ & $-C 7$ & $104.87(15)$ & Al1 & $-\mathrm{C} 2$ & $-\mathrm{H} 21$ & $123(2)$ \\
\hline C5 & $-A 12$ & $-\mathrm{C} 8$ & $106.71(14)$ & Al1 & $-\mathrm{C} 2$ & $-\mathrm{H} 22$ & $92(2)$ \\
\hline $\mathrm{C} 6$ & -Al2 & $-C 7$ & $106.73(16)$ & Al1 & $-\mathrm{C} 2$ & $-\mathrm{H} 23$ & $120.7(17)$ \\
\hline C6 & $-A 12$ & $-\mathrm{C} 8$ & $108.58(15)$ & $\mathrm{H} 21$ & $-\mathrm{C} 2$ & $-\mathrm{H} 22$ & $104(3)$ \\
\hline C7 & -Al2 & $-\mathrm{C} 8$ & $119.62(16)$ & $\mathrm{H} 21$ & $-\mathrm{C2}$ & $-\mathrm{H} 23$ & $108(3)$ \\
\hline La & -01 & $-\mathrm{CO}$ & $168.21(18)$ & $\mathrm{H} 22$ & $-\mathrm{C} 2$ & $-\mathrm{H} 23$ & $104(3)$ \\
\hline La_a & -02 & $-C 9$ & $123.18(18)$ & Al1 & $-\mathrm{C3}$ & $-H 31$ & $112(2)$ \\
\hline La & $-C_{1}$ & -Al1 & $85.46(10)$ & Al1 & $-\mathrm{C3}$ & $-H 32$ & $111(3)$ \\
\hline La & $-\mathrm{C} 2$ & -Al1 & $85.65(10)$ & Al1 & $-\mathrm{C3}$ & - H33 & $113(2)$ \\
\hline La & $-\mathrm{C5}$ & $-A 12$ & $85.12(10)$ & H31 & $-\mathrm{C3}$ & $-H 32$ & $109(4)$ \\
\hline La & $-\mathrm{C} 6$ & $-A 12$ & $85.24(13)$ & H31 & $-\mathrm{C3}$ & - H33 & $107(3)$ \\
\hline 01 & $-C 9$ & -02 & $120.2(3)$ & H32 & $-\mathrm{C3}$ & - H33 & $106(3)$ \\
\hline 01 & $-\mathrm{CO}$ & $-\mathrm{C} 10$ & $117.0(2)$ & Al1 & $-\mathrm{C} 4$ & $-\mathrm{H} 41$ & $115(3)$ \\
\hline 02 & $-C 9$ & $-\mathrm{C} 10$ & $122.7(2)$ & Al1 & $-\mathrm{C} 4$ & $-\mathrm{H} 42$ & $116(3)$ \\
\hline $\mathrm{C9}$ & $-\mathrm{C} 10$ & $-C 11$ & $119.3(2)$ & Al1 & $-\mathrm{C} 4$ & $-\mathrm{H} 43$ & $111(3)$ \\
\hline $\mathrm{CO}$ & - C10 & $-\mathrm{C} 15$ & $120.11(19)$ & $\mathrm{H} 41$ & $-\mathrm{C} 4$ & $-\mathrm{H} 42$ & $105(4)$ \\
\hline C11 & $-\mathrm{C} 10$ & $-C 15$ & $120.3(2)$ & H41 & $-\mathrm{C} 4$ & $-\mathrm{H} 43$ & $108(4)$ \\
\hline C10 & $-C 11$ & $-\mathrm{C} 12$ & $117.3(2)$ & $\mathrm{H} 42$ & $-\mathrm{C} 4$ & $-\mathrm{H} 43$ & $100(4)$ \\
\hline C10 & $-C 11$ & - C16 & $124.4(2)$ & La & $-\mathrm{C5}$ & $-H 51$ & $80.2(18)$ \\
\hline C12 & $-C 11$ & $-\mathrm{C} 16$ & $118.30(19)$ & La & $-\mathrm{C5}$ & $-\mathrm{H} 52$ & $177.4(18)$ \\
\hline C11 & $-C 12$ & - C13 & $124.0(2)$ & La & $-\mathrm{C5}$ & $-H 53$ & $77.3(15)$ \\
\hline C12 & $-C 13$ & - C14 & $116.9(2)$ & Al2 & $-\mathrm{C5}$ & $-H 51$ & $120(2)$ \\
\hline C12 & $-C 13$ & $-C 20$ & $119.7(2)$ & Al2 & $-\mathrm{C5}$ & $-H 52$ & $92.4(18)$ \\
\hline C14 & $-C 13$ & $-\mathrm{C} 20$ & $123.4(2)$ & Al2 & $-\mathrm{C5}$ & $-H 53$ & $128.2(15)$ \\
\hline C13 & - C14 & $-\mathrm{C} 15$ & $123.3(2)$ & H51 & $-\mathrm{C5}$ & $-\mathrm{H} 52$ & $102(2)$ \\
\hline C10 & - C15 & $-C 14$ & $117.73(19)$ & H51 & $-\mathrm{C5}$ & $-\mathrm{H} 53$ & $104(2)$ \\
\hline
\end{tabular}


Table S18 (cont.) - Bond Angles (Degrees) for: Compound 9b

\begin{tabular}{|c|c|c|c|c|c|c|c|}
\hline C10 & - C15 & $-C 24$ & $127.2(2)$ & H52 & $-\mathrm{C5}$ & $-H 53$ & $104(2)$ \\
\hline C14 & $-C 15$ & $-\mathrm{C} 24$ & $115.0(2)$ & $\mathrm{La}$ & $-\mathrm{C} 6$ & $-H 61$ & $80(2)$ \\
\hline C11 & - C16 & - C17 & $108.95(19)$ & $\mathrm{La}$ & $-\mathrm{C6}$ & $-\mathrm{H} 62$ & $177.7(17)$ \\
\hline C11 & $-\mathrm{C} 16$ & - C18 & $112.63(19)$ & $\mathrm{La}$ & $-\mathrm{C} 6$ & $-\mathrm{H} 63$ & $83(3)$ \\
\hline C11 & - C16 & - C19 & $112.2(2)$ & Al2 & $-\mathrm{C} 6$ & $-H 61$ & $126(2)$ \\
\hline C17 & - C16 & $-\mathrm{C} 18$ & $110.9(2)$ & Al2 & $-\mathrm{C} 6$ & $-\mathrm{H} 62$ & $94.9(18)$ \\
\hline C17 & $-\mathrm{C} 16$ & - C19 & $106.6(2)$ & Al2 & $-\mathrm{C} 6$ & $-\mathrm{H} 63$ & $119(2)$ \\
\hline C18 & - C16 & - C19 & $105.5(2)$ & H61 & $-\mathrm{C} 6$ & $-\mathrm{H} 62$ & $98(3)$ \\
\hline C13 & $-C 20$ & $-C 21$ & $110.6(3)$ & H61 & $-\mathrm{C} 6$ & $-\mathrm{H} 63$ & $110(3)$ \\
\hline C13 & $-\mathrm{C} 20$ & $-\mathrm{C} 22$ & $112.6(2)$ & $\mathrm{H} 62$ & $-\mathrm{C} 6$ & $-\mathrm{H} 63$ & $99(3)$ \\
\hline C13 & $-\mathrm{C} 20$ & $-C 23$ & $108.6(3)$ & Al2 & $-C 7$ & $-\mathrm{H} 71$ & $114(2)$ \\
\hline Al2 & $-C 7$ & $-\mathrm{H} 72$ & $111(2)$ & $\mathrm{C} 20$ & $-C 22$ & $-H 221$ & 109.51 \\
\hline Al2 & $-C 7$ & $-\mathrm{H} 73$ & $115(3)$ & $\mathrm{C} 20$ & $-C 22$ & $-H 222$ & 109.41 \\
\hline H71 & $-C 7$ & $-\mathrm{H} 72$ & $107(3)$ & $\mathrm{C} 20$ & $-C 22$ & $-H 223$ & 109.43 \\
\hline H71 & $-C 7$ & $-\mathrm{H} 73$ & $103(3)$ & $\mathrm{H} 221$ & $-C 22$ & $-\mathrm{H} 222$ & 109.53 \\
\hline $\mathrm{H} 72$ & $-C 7$ & $-\mathrm{H} 73$ & $107(3)$ & $\mathrm{H} 221$ & $-C 22$ & $-H 223$ & 109.47 \\
\hline Al2 & $-\mathrm{C} 8$ & $-\mathrm{H} 81$ & $116.5(18)$ & $\mathrm{H} 222$ & $-C 22$ & $-\mathrm{H} 223$ & 109.48 \\
\hline Al2 & $-\mathrm{C} 8$ & $-\mathrm{H} 82$ & $111.4(19)$ & $\mathrm{C} 20$ & $-C 23$ & $-H 231$ & 109.54 \\
\hline Al2 & $-\mathrm{C} 8$ & $-\mathrm{H} 83$ & $113.2(19)$ & $\mathrm{C} 20$ & $-C 23$ & $-H 232$ & 109.45 \\
\hline H81 & $-\mathrm{C} 8$ & $-\mathrm{H} 82$ & $107(3)$ & $\mathrm{C} 20$ & $-C 23$ & $-H 233$ & 109.52 \\
\hline H81 & $-\mathrm{C} 8$ & $-\mathrm{H} 83$ & $103(3)$ & $\mathrm{H} 231$ & $-C 23$ & $-H 232$ & 109.40 \\
\hline \multirow[t]{2}{*}{ H82 } & $-\mathrm{C} 8$ & $-\mathrm{H} 83$ & $105(3)$ & $\mathrm{H} 231$ & $-C 23$ & $-H 233$ & 109.57 \\
\hline & & & & $\mathrm{H} 232$ & $-C 23$ & $-H 233$ & 109.35 \\
\hline C11 & $-\mathrm{C} 12$ & $-\mathrm{H} 121$ & 117.97 & C24 & $-C 25$ & $-H 251$ & 109.51 \\
\hline C13 & $-\mathrm{C} 12$ & $-\mathrm{H} 121$ & 118.08 & $\mathrm{C} 24$ & $-C 25$ & $-H 252$ & 109.49 \\
\hline C13 & - C14 & $-\mathrm{H} 141$ & 118.40 & C24 & $-C 25$ & $-H 253$ & 109.43 \\
\hline C15 & $-C_{14}$ & $-\mathrm{H} 141$ & 118.31 & $\mathrm{H} 251$ & $-C 25$ & $-H 252$ & 109.38 \\
\hline C16 & $-\mathrm{C} 17$ & $-\mathrm{H} 171$ & 109.44 & $\mathrm{H} 251$ & $-C 25$ & $-H 253$ & 109.47 \\
\hline C16 & $-\mathrm{C} 17$ & $-\mathrm{H} 172$ & 109.43 & $\mathrm{H} 252$ & $-C 25$ & $-H 253$ & 109.54 \\
\hline C16 & $-\mathrm{C} 17$ & $-\mathrm{H} 173$ & 109.50 & C24 & $-C 26$ & $-H 261$ & 109.50 \\
\hline $\mathrm{H} 171$ & $-\mathrm{C} 17$ & $-\mathrm{H} 172$ & 109.45 & $\mathrm{C} 24$ & $-C 26$ & $-H 262$ & 109.43 \\
\hline $\mathrm{H} 171$ & $-\mathrm{C} 17$ & $-\mathrm{H} 173$ & 109.52 & C24 & $-C 26$ & $-H 263$ & 109.48 \\
\hline $\mathrm{H} 172$ & $-\mathrm{C} 17$ & $-\mathrm{H} 173$ & 109.49 & $\mathrm{H} 261$ & $-C 26$ & $-\mathrm{H} 262$ & 109.42 \\
\hline C16 & - C18 & - H181 & 109.46 & H261 & $-C 26$ & $-H 263$ & 109.49 \\
\hline C16 & $-\mathrm{C} 18$ & - H182 & 109.48 & H262 & $-C 26$ & $-H 263$ & 109.50 \\
\hline C16 & - C18 & - H183 & 109.47 & C24 & $-C 27$ & $-H 271$ & 109.41 \\
\hline H181 & $-\mathrm{C} 18$ & $-\mathrm{H} 182$ & 109.48 & C24 & $-C 27$ & $-\mathrm{H} 272$ & 109.48 \\
\hline H181 & - C18 & - H183 & 109.41 & C24 & $-C 27$ & $-H 273$ & 109.50 \\
\hline H182 & - C18 & - H183 & 109.53 & $\mathrm{H} 271$ & $-C 27$ & $-H 272$ & 109.51 \\
\hline C16 & - C19 & - H191 & 109.47 & $\mathrm{H} 271$ & $-C 27$ & $-\mathrm{H} 273$ & 109.47 \\
\hline C16 & - C19 & - H192 & 109.44 & $\mathrm{H} 272$ & $-C 27$ & $-\mathrm{H} 273$ & 109.46 \\
\hline C16 & - C19 & $-\mathrm{H} 193$ & 109.47 & & & & \\
\hline H191 & - C19 & - H192 & 109.49 & & & & \\
\hline H191 & - C19 & - H193 & 109.51 & & & & \\
\hline H192 & - C19 & - H193 & 109.45 & & & & \\
\hline $\mathrm{C} 20$ & $-C 21$ & $-H 211$ & 109.44 & & & & \\
\hline $\mathrm{C} 20$ & $-C 21$ & $-\mathrm{H} 212$ & 109.48 & & & & \\
\hline C20 & $-C 21$ & $-H 213$ & 109.47 & & & & \\
\hline $\mathrm{H} 211$ & $-C 21$ & $-\mathrm{H} 212$ & 109.60 & & & & \\
\hline $\mathrm{H} 211$ & $-C 21$ & $-\mathrm{H} 213$ & 109.37 & & & & \\
\hline $\mathrm{H} 212$ & $-C 21$ & $-\mathrm{H} 213$ & 109.46 & & & & \\
\hline
\end{tabular}

Translation of Symmetry Code to Equiv.Pos

$a=[2555.00]=-x, y, 0.5-z$ 


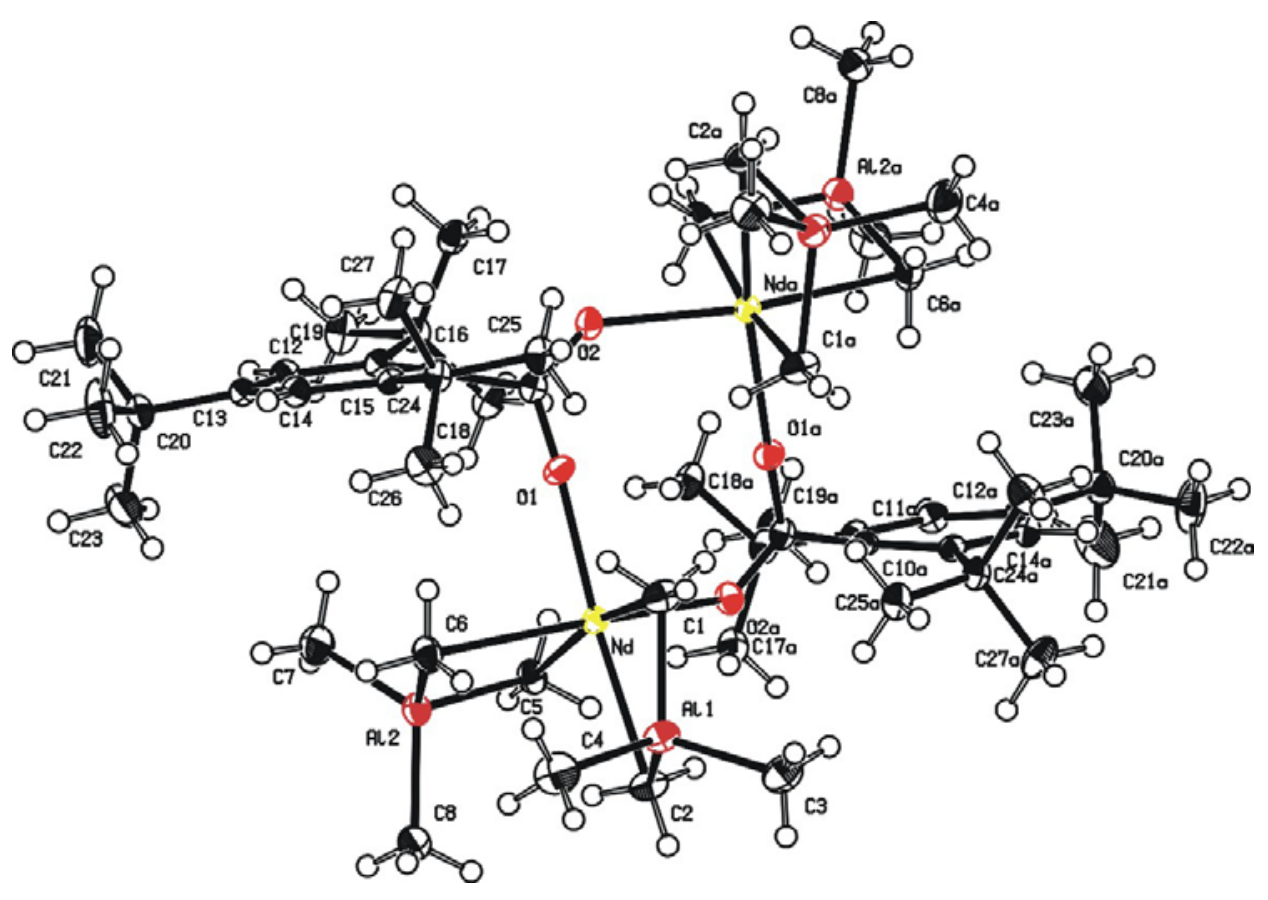

Molecular structure of $\left\{\mathrm{Nd}\left(\mathrm{O}_{2} \mathrm{CAr}{ }^{\mathrm{tBu}}\right)\left[(\mu-\mathrm{Me})_{2} \mathrm{AlMe}_{2}\right]_{2}\right\}_{2}$ (9c)

Table S19 - Crystal Data and Details of the Structure Determination for: Compound 9c

Crystal Data

Formula

Formula weight

crystal System

Space group

$a, b, c$ [Angstrom]

alpha, beta, gamma [deg]

$\mathrm{V}[\mathrm{Ang} *$ * 3$]$

Z

$D($ calc $)\left[\mathrm{g} / \mathrm{cm}^{*} * 3\right]$

$\mathrm{Mu}($ MoKa $)[/ \mathrm{mm}]$

$\mathrm{F}(\odot \odot \odot)$

crystal size [mm]
C54 H106 Al4 Nd2 04

1215.79

Monoclinic

$\mathrm{C} 2 / \mathrm{C}$

$\begin{array}{rr}23.5611(2) & 19.4031(1) \\ 90 & 113.5901(3)\end{array}$

(No. 15)

$15.2363(1)$

6383. 32(8)

1.265

1.700

2536

$0.18 \times 0.18 \times 0.51$

Data Collection

Temperature (K)

Radiation [Angstrom]

Theta Min-Max [Deg]

Dataset

Tot., Uniq. Data, R(int)

Observed data $[I>2.0$ sigma(I)]

Refinement

Nref, Npar

$\mathrm{R}$ ，WR2， $\mathrm{S}$

$\begin{array}{lr}R, W R 2, S & 0.0179,0.0415,1.02 \\ W^{\prime}=1 /\left[\backslash S^{\wedge} 2^{\wedge}\left(F 0^{\wedge} 2^{\wedge}\right)+(\odot .0160 P)^{\wedge} 2^{\wedge}+10.4851 P\right] & \text { Where } P=\left(F 0^{\wedge} 2^{\wedge}+2 F^{\wedge} 2^{\wedge}\right) /\end{array}$

Max. and Av. Shift/Error

Min. and Max. Resd. Dens. [e/Ang^3]

$$
\begin{array}{rr}
\text { MoKa } & 0.71073 \\
-28: 28 ;-23: 23 ; & 25.3 \\
75409, & 5856, \quad 0.041 \\
&
\end{array}
$$


Table S20 - Final Coordinates and Equivalent Isotropic Displacement Parameters of the non-Hydrogen atoms for: Compound 9c

\begin{tabular}{|c|c|c|c|c|}
\hline Atom & $\mathrm{x}$ & $\mathrm{y}$ & Z & $\mathrm{U}(\mathrm{eq}) \quad\left[\mathrm{Ang} \wedge^{\wedge}\right.$ \\
\hline--- & -- & --- & --- & -------- \\
\hline $\mathrm{Nd}$ & $-0.03196(1)$ & $\odot .24785$ (1) & ๑. $06828(1)$ & $0.0144(1)$ \\
\hline Al1 & $-0.07777(3)$ & ๑.39454(3) & $-0.02867(4)$ & $0.0219(2)$ \\
\hline Al2 & $-0.00666(3)$ & $0.15015(3)$ & $-0.07960(4)$ & 0.0201(2) \\
\hline 01 & ๑.05378(6) & $0.23136(7)$ & ๑.20630(9) & $0.0199(4)$ \\
\hline 02 & ๑.10312(6) & $\odot .22043(6)$ & ๑. 36302(9) & $0.0185(4)$ \\
\hline C1 & $-0.01638(10)$ & $0.37884(11)$ & $0.11183(15)$ & $0.0253(6)$ \\
\hline $\mathrm{C} 2$ & $-0.12256(10)$ & $0.30163(11)$ & $-0.07937(15)$ & $0.0248(6)$ \\
\hline C3 & $-0.14163(11)$ & $0.45860(12)$ & $-0.02567(19)$ & ๑.0319(7) \\
\hline $\mathrm{C} 4$ & $-0.02922(13)$ & $0.42260(14)$ & $-0.1020(2)$ & ๑. . $0379(8)$ \\
\hline C5 & $-0.06221(10)$ & $0.12519(10)$ & $-0.00701(16)$ & $0.0216(6)$ \\
\hline C6 & $0.04133(10)$ & $0.24031(11)$ & $-0.02354(16)$ & $0.0250(7)$ \\
\hline C7 & 0.05523(12) & $0.07602(13)$ & $-0.04798(19)$ & ๑. . $0327(8)$ \\
\hline $\mathrm{C} 8$ & $-0.06336(10)$ & $0.16729(13)$ & $-0.21427(15)$ & $0.0274(7)$ \\
\hline $\mathrm{C9}$ & $0.10281(8)$ & ๑.21807(9) & $0.28019(13)$ & $0.0157(5)$ \\
\hline C10 & 0.15880 (8) & $\odot .19789(9)$ & $0.26088(12)$ & $0.0148(5)$ \\
\hline C11 & ๑.17071(8) & ๑.12626(9) & $0.25328(13)$ & $0.0165(5)$ \\
\hline C12 & 0.21061(9) & $0.10949(10)$ & $0.20985(13)$ & 0.0193(6) \\
\hline C13 & $0.24077(8)$ & $0.15809(9)$ & $0.17663(13)$ & $0.0175(5)$ \\
\hline C14 & ○.23299(9) & $0.22645(10)$ & $0.19393(13)$ & 0.0188(5) \\
\hline C15 & $0.19293(8)$ & $\odot .24852(9)$ & $0.23664(12)$ & ๑. .0156(5) \\
\hline C16 & $0.14519(8)$ & ๑.06757(9) & $0.29608(14)$ & $0.0202(6)$ \\
\hline C17 & $0.17310(10)$ & $0.07511(11)$ & $0.40585(15)$ & $0.0233(6)$ \\
\hline C18 & $\odot .07391(9)$ & $0.06647(11)$ & $0.25572(17)$ & $0.0236(6)$ \\
\hline C19 & $0.16445(11)$ & $-0.00410(10)$ & $0.27443(18)$ & $0.0276(7)$ \\
\hline $\mathrm{C} 20$ & $\odot .28010(9)$ & $0.13361(10)$ & $0.12232(14)$ & $0.0225(6)$ \\
\hline C21 & $0.32285(14)$ & $0.07382(16)$ & $0.1767(2)$ & $0.0474(10)$ \\
\hline $\mathrm{C} 22$ & $0.32101(13)$ & $0.19060(14)$ & $0.1107(2)$ & $0.0438(9)$ \\
\hline C23 & $0.23651(12)$ & $0.10759(15)$ & $0.02378(18)$ & $0.0387(8)$ \\
\hline $\mathrm{C} 24$ & $0.19386(8)$ & $\odot .32713(9)$ & $0.25591(13)$ & 0.0184(5) \\
\hline $\mathrm{C} 25$ & $0.15575(10)$ & $0.35138(10)$ & $0.31145(16)$ & $0.0224(6)$ \\
\hline $\mathrm{C} 26$ & $0.17127(11)$ & $0.36683(11)$ & $0.16091(16)$ & $0.0291(7)$ \\
\hline C27 & ๑. 26118(10) & $0.34829(11)$ & $0.31834(18)$ & ๑.0287(7) \\
\hline
\end{tabular}

$U(e q)=1 / 3$ of the trace of the orthogonalized $U$ Tensor

Table S21 - Hydrogen Atom Positions and Isotropic Displacement Parameters for: Compound 9c

\begin{tabular}{|c|c|c|c|c|}
\hline Atom & $\mathrm{X}$ & $\mathrm{y}$ & z & $\mathrm{U}($ iso $) \quad\left[\mathrm{Ang}^{\wedge} 2\right]$ \\
\hline--- & -- & -- & -- & ---- \\
\hline H11 & $0.0243(12)$ & $0.3620(13)$ & $0.1285(18)$ & $0.047(7)$ \\
\hline $\mathrm{H} 12$ & $-0.0291(11)$ & $0.3603(12)$ & $0.1615(17)$ & $0.040(7)$ \\
\hline H13 & $-0.0115(10)$ & $0.4268(13)$ & $0.1251(17)$ & $0.040(7)$ \\
\hline $\mathrm{H} 21$ & $-0.1072(10)$ & $0.2667(12)$ & $-0.1077(16)$ & $0.027(6)$ \\
\hline $\mathrm{H} 22$ & $-0.1546(10)$ & $0.3236(12)$ & $-0.1334(16)$ & $0.033(6)$ \\
\hline H23 & $-0.1462(11)$ & ๑. $2787(13)$ & $-0.0455(17)$ & $0.039(6)$ \\
\hline H31 & $-0.1590(12)$ & $0.4426(14)$ & $0.018(2)$ & ๑. $055(8)$ \\
\hline H32 & $-0.1718(13)$ & $0.4629(14)$ & $-0.087(2)$ & $0.056(8)$ \\
\hline H33 & $-0.1240(12)$ & $0.5043(14)$ & $-0.0020(19)$ & ๑. 053(8) \\
\hline H41 & $-0.0273(13)$ & ๑. 3896(16) & $-0.148(2)$ & ๑.065(9) \\
\hline H42 & ๑.0086(15) & $0.4345(16)$ & $-0.066(2)$ & $0.067(10)$ \\
\hline $\mathrm{H} 43$ & $-0.0477(12)$ & $0.4625(15)$ & $-0.143(2)$ & $0.057(8)$ \\
\hline H51 & $-0.0475(10)$ & $0.1144(12)$ & $\odot .0560(17)$ & $0.032(6)$ \\
\hline H52 & $-0.0720(10)$ & $0.0844(13)$ & $-0.0365(16)$ & ๑.031(6) \\
\hline H53 & $-0.1019(10)$ & $0.1460(11)$ & $-0.0181(15)$ & $0.029(6)$ \\
\hline H61 & $0.0236(11)$ & ๑. 2848(14) & $-0.0395(17)$ & $0.039(7)$ \\
\hline H62 & ๑.0658(12) & $0.2372(12)$ & $-0.0606(19)$ & $0.041(7)$ \\
\hline H63 & ๑.0717(12) & ๑.2406(11) & $0.0410(19)$ & $0.039(7)$ \\
\hline
\end{tabular}


Table S21 (cont.) - Hydrogen Atom Positions and Isotropic Displacement Parameters for: Compound 9c

\begin{tabular}{|c|c|c|c|c|}
\hline Atom & $\mathrm{x}$ & $\mathrm{y}$ & Z & $\mathrm{U}($ iso $)\left[\mathrm{Ang}^{\wedge}\right.$ \\
\hline---- & --- & -- & -- & $-\ldots$ \\
\hline H71 & ๑.0781(13) & $\odot .0729(14)$ & $0.019(2)$ & $0.055(8)$ \\
\hline H72 & $0.0839(14)$ & ๑.0837(15) & $-0.075(2)$ & ๑.064(9) \\
\hline H73 & ๑.0377(12) & ๑.0335(16) & $-0.0662(19)$ & ๑.059(9) \\
\hline H81 & $-0.1067(12)$ & $0.1707(13)$ & $-0.2233(17)$ & $\odot .048(7)$ \\
\hline H82 & $-0.0587(11)$ & $0.1344(13)$ & $-0.2526(18)$ & $0.043(7)$ \\
\hline H83 & $-0.0556(11)$ & $0.2095(15)$ & $-0.2375(18)$ & $0.046(7)$ \\
\hline $\mathrm{H} 121$ & $0.2178(8)$ & $0.0643(10)$ & $0.2024(13)$ & $0.014(5)$ \\
\hline H141 & $0.2549(10)$ & ๑. 2609(10) & $0.1745(14)$ & $0.017(5)$ \\
\hline H171 & $0.1655(9)$ & $0.1194(11)$ & $0.4265(14)$ & $0.022(5)$ \\
\hline $\mathrm{H} 172$ & $\odot .2180(10)$ & $0.0685(10)$ & $0.4312(15)$ & $0.025(5)$ \\
\hline $\mathrm{H} 173$ & $0.1576(10)$ & ๑. . $413(11)$ & $\odot .4350(15)$ & $0.027(6)$ \\
\hline H181 & $0.0556(9)$ & $0.1041(11)$ & $0.2710(14)$ & $0.018(5)$ \\
\hline H182 & $0.0600(9)$ & $\odot .0260(11)$ & ๑. $2797(15)$ & $0.025(5)$ \\
\hline H183 & $0.0580(10)$ & ๑.0636(11) & $0.1905(17)$ & $0.028(6)$ \\
\hline H191 & $0.2101(11)$ & $-0.0091(13)$ & $0.3010(17)$ & $0.040(7)$ \\
\hline H192 & $0.1489(10)$ & $-0.0136(11)$ & ๑. 2064(17) & $0.027(6)$ \\
\hline H193 & $0.1481(10)$ & $-0.0382(12)$ & $0.3042(16)$ & $0.031(6)$ \\
\hline $\mathrm{H} 211$ & $0.3502(16)$ & $\odot .0890(18)$ & $0.246(3)$ & $0.095(12)$ \\
\hline $\mathrm{H} 212$ & $0.3492(11)$ & $0.0608(13)$ & $0.1440(18)$ & $0.044(7)$ \\
\hline $\mathrm{H} 213$ & $0.2955(15)$ & ๑. $0358(17)$ & $0.168(2)$ & $0.074(11)$ \\
\hline $\mathrm{H} 221$ & $0.3487(13)$ & $0.2066(15)$ & $0.173(2)$ & ๑. .059( 8) \\
\hline $\mathrm{H} 222$ & ๑.2970(14) & ๑. 2299(17) & $0.074(2)$ & $0.065(9)$ \\
\hline $\mathrm{H} 223$ & $0.3453(11)$ & $0.1722(12)$ & $0.0774(17)$ & $0.042(7)$ \\
\hline H231 & $0.2097(11)$ & ๑.069๑(13) & ๑. $0299(17)$ & $0.046(7)$ \\
\hline $\mathrm{H} 232$ & $0.2598(11)$ & $0.0926(13)$ & $-0.0140(17)$ & $0.041(7)$ \\
\hline $\mathrm{H} 233$ & $\odot .2132(10)$ & $0.1476(12)$ & $-0.0097(16)$ & $0.028(6)$ \\
\hline H251 & $0.1686(9)$ & $0.3282(11)$ & $0.3725(16)$ & $0.025(5)$ \\
\hline H252 & $0.1117(9)$ & $0.3443(10)$ & ๑.2751(14) & $0.016(5)$ \\
\hline H253 & $0.1612(10)$ & ๑. 3981(12) & ๑. 3224(15) & $0.030(6)$ \\
\hline $\mathrm{H} 261$ & $0.1957(12)$ & $0.3567(13)$ & $0.1239(18)$ & $0.046(7)$ \\
\hline $\mathrm{H} 262$ & $0.1715(10)$ & $0.4142(13)$ & $0.1713(16)$ & $0.035(6)$ \\
\hline $\mathrm{H} 263$ & $0.1303(11)$ & $0.3536(11)$ & $0.1212(16)$ & $0.029(6)$ \\
\hline $\mathrm{H} 271$ & $0.2761(11)$ & $0.3218(13)$ & $0.3812(18)$ & $0.044(7)$ \\
\hline $\mathrm{H} 272$ & $0.2628(10)$ & $0.3970(12)$ & $0.3322(15)$ & $0.031(6)$ \\
\hline $\mathrm{H} 273$ & ๑.2881(11) & $\odot .3378(12)$ & ๑. 2884(17) & $0.040(7)$ \\
\hline
\end{tabular}

The Temperature Factor has the Form of $\operatorname{Exp}(-\mathrm{T})$ Where

$\mathrm{T}=8^{*}(\mathrm{Pi} * 2) * \mathrm{U}^{*}(\operatorname{Sin}($ Theta $) /$ Lambda $) * * 2$ for Isotropic Atoms

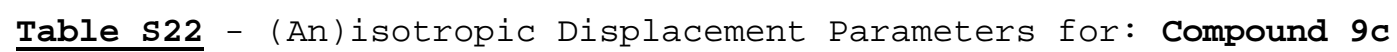

\begin{tabular}{|c|c|c|c|c|c|c|}
\hline Atom & $\mathrm{U}(1,1)$ or & $\mathrm{U}(2,2)$ & $\mathrm{U}(3,3)$ & $U(2,3)$ & $U(1,3)$ & $U(1,2)$ \\
\hline$-\ldots$ & ----- & $\ldots \ldots$ & - _- & - & - & - \\
\hline $\mathrm{Nd}$ & $0.0136(1)$ & $0.0138(1)$ & $0.0158(1)$ & $0.0005(1)$ & $0.0059(1)$ & $-0.0001(1)$ \\
\hline Al1 & $0.0213(3)$ & $0.0171(3)$ & ○. $0243(3)$ & $\odot .0042(2)$ & 0.0061(2) & $-0.0010(2)$ \\
\hline Al2 & $0.0209(3)$ & $0.0196(3)$ & $0.0214(3)$ & $-0.0022(2)$ & $0.0101(2)$ & $0.0004(2)$ \\
\hline 01 & $0.0150(6)$ & $0.0218(7)$ & $0.0209(7)$ & $0.0045(5)$ & $0.0050(6)$ & $0.0029(5)$ \\
\hline 02 & 0.0194(7) & $0.0173(6)$ & $0.0214(7)$ & ๑.0010(5) & $0.0109(6)$ & ๑.0025(5) \\
\hline C1 & $0.0258(11)$ & $0.0168(10)$ & $0.0275(12)$ & $0.0002(9)$ & $0.0045(9)$ & $-0.0002(8)$ \\
\hline $\mathrm{C} 2$ & 0.0231(11) & $0.0214(10)$ & $0.0241(11)$ & ๑.0023(9) & ๑.0034(9) & $-0.0007(8)$ \\
\hline C3 & ๑.0319(12) & $0.0253(12)$ & $0.0354(14)$ & $0.0070(10)$ & $0.0103(11)$ & ○.0057(9) \\
\hline $\mathrm{C} 4$ & ๑.0356(14) & ๑.0365(14) & $0.0415(15)$ & ๑.0067(12) & ๑.0153(12) & $-0.0079(11)$ \\
\hline $\mathrm{C} 5$ & $0.0271(11)$ & 0.0181(10) & $0.0234(11)$ & $0.0006(9)$ & $0.0140(9)$ & ๑. .0015( 8 ) \\
\hline C6 & $0.0238(11)$ & $0.0273(12)$ & $0.0280(12)$ & $-0.0041(9)$ & $0.0146(10)$ & $-0.0044(9)$ \\
\hline C7 & $0.0345(13)$ & ๑. .0305(13) & $0.0344(14)$ & $-0.0001(10)$ & $0.0153(11)$ & $0.0082(10)$ \\
\hline $\mathrm{C} 8$ & $0.0257(12)$ & $0.0337(13)$ & $0.0241(11)$ & $-0.0024(10)$ & $0.0112(9)$ & $-0.0005(9)$ \\
\hline
\end{tabular}


Table s22 (cont.) - (An)isotropic Displacement Parameters for: Compound 9c

\begin{tabular}{|c|c|c|c|c|c|c|}
\hline Atom & $U(1,1)$ or & $U(2,2)$ & $U(3,3)$ & $U(2,3)$ & $U(1,3)$ & $U(1,2)$ \\
\hline--- & ----- & - & - & ---- & - & - \\
\hline $\mathrm{C9}$ & $0.0176(9)$ & ๑.0०91( 8 ) & $0.0215(10)$ & $0.0009(7)$ & ๑. 0089 ( 8) & $-0.0009(7)$ \\
\hline C10 & 0.0139(9) & $0.0168(9)$ & $0.0125(9)$ & $-0.0004(7)$ & $0.0040(7)$ & $0.0002(7)$ \\
\hline C11 & $0.0139(9)$ & $0.0171(9)$ & $0.0176(9)$ & $0.0002(7)$ & $0.0054(7)$ & $-0.0001(7)$ \\
\hline C12 & $0.0203(10)$ & 0.0152(9) & $0.0214(10)$ & $-0.0022(8)$ & $0.0074(8)$ & $0.0007(7)$ \\
\hline C13 & $0.0149(9)$ & $0.0216(9)$ & $0.0158(9)$ & $-0.0017(7)$ & $0.0059(7)$ & $0.0003(7)$ \\
\hline C14 & $0.0178(9)$ & $0.0193(9)$ & $0.0204(10)$ & $0.0002(8)$ & $0.0088(8)$ & $-0.0025(8)$ \\
\hline C15 & $0.0143(9)$ & $0.0158(9)$ & ๑. .0155(9) & $0.0010(7)$ & $0.0046(7)$ & $0.0012(7)$ \\
\hline C16 & 0.0196(10) & 0.0164(9) & $0.0265(10)$ & $0.0021(8)$ & 0.0111( 8) & $0.0008(7)$ \\
\hline C17 & $0.0221(11)$ & $0.0219(11)$ & $0.0278(11)$ & ๑.0084(9) & 0.0121(9) & $0.0052(8)$ \\
\hline C18 & $0.0208(10)$ & $0.0194(10)$ & $0.0320(13)$ & $0.0026(9)$ & $0.0119(9)$ & $-0.0023(8)$ \\
\hline C19 & $0.0301(12)$ & $0.0159(10)$ & $0.0424(14)$ & ๑.0005(9) & $0.0203(11)$ & $-0.0010(8)$ \\
\hline $\mathrm{C} 20$ & $0.0237(10)$ & $0.0235(10)$ & $0.0240(10)$ & $-0.0042(8)$ & $0.0136(8)$ & $-0.0005(8)$ \\
\hline C21 & ๑.0499(16) & ๑.0569(18) & 0.0498(17) & $0.0066(14)$ & $\odot .0350(15)$ & $0.0260(14)$ \\
\hline $\mathrm{C} 22$ & $0.0436(15)$ & ๑.0422(15) & $0.0660(19)$ & $-0.0247(14)$ & $0.0435(15)$ & $-0.0171(12)$ \\
\hline C23 & $0.0383(13)$ & $0.0528(16)$ & $0.0315(13)$ & $-0.0133(12)$ & $0.0208(11)$ & $-0.0098(12)$ \\
\hline C24 & ๑. .0184(9) & $0.0144(9)$ & $0.0243(10)$ & $-0.0003(7)$ & ๑. . 0106( 8) & $-0.0 \odot \odot 3(7)$ \\
\hline $\mathrm{C} 25$ & $0.0248(11)$ & 0.0156(10) & ๑. .0301(12) & $-0.0022(9)$ & $0.0144(9)$ & $-0.0012(8)$ \\
\hline C26 & $0.0399(14$ & $0.0186(11)$ & $0.0319(12)$ & $0.0036(9)$ & $0.0177(11)$ & $0.0014(9)$ \\
\hline $\mathrm{C} 27$ & $0.0213(11)$ & $0.0201(11)$ & $0.0444(14)$ & $-0.0089(10)$ & $0.0128(10)$ & $-0.0038(8)$ \\
\hline
\end{tabular}

The Temperature Factor has the Form as given in Table S4

Table S23 - Bond Distances (Angstrom) for: Compound 9c

\begin{tabular}{|c|c|c|c|c|c|}
\hline $\mathrm{Nd}$ & -01 & $2.2815(13)$ & $\mathrm{C} 4$ & $-\mathrm{H} 41$ & $0.96(3)$ \\
\hline $\mathrm{Nd}$ & $-\mathrm{C1}$ & $2.615(2)$ & $\mathrm{C} 4$ & $-\mathrm{H} 42$ & $0.87(3)$ \\
\hline $\mathrm{Nd}$ & $-\mathrm{C} 2$ & $2.620(2)$ & $\mathrm{C} 4$ & $-\mathrm{H} 43$ & $0.98(3)$ \\
\hline Nd & $-\mathrm{C5}$ & $2.614(2)$ & C5 & $-H 51$ & $0.91(2)$ \\
\hline $\mathrm{Nd}$ & $-\mathrm{C} 6$ & $2.626(2)$ & C5 & $-\mathrm{H} 52$ & $0.89(2)$ \\
\hline Nd & $-02 \_a$ & $2.3652(15)$ & C5 & $-H 53$ & $0.97(2)$ \\
\hline Al1 & $-C 1$ & $2.071(2)$ & C6 & $-\mathrm{H} 61$ & $0.95(3)$ \\
\hline Al1 & $-\mathrm{C} 2$ & $2.076(2)$ & C6 & $-\mathrm{H} 62$ & $0.96(3)$ \\
\hline Al1 & $-\mathrm{C3}$ & $1.966(3)$ & $\mathrm{C} 6$ & $-H 63$ & $0.96(3)$ \\
\hline Al1 & $-\mathrm{C} 4$ & $1.969(3)$ & C7 & $-\mathrm{H} 71$ & $0.95(3)$ \\
\hline Al2 & $-\mathrm{C5}$ & $2.081(3)$ & C7 & $-\mathrm{H} 72$ & $0.93(3)$ \\
\hline Al2 & $-\mathrm{C} 6$ & $2.073(2)$ & C7 & $-\mathrm{H} 73$ & $0.92(3)$ \\
\hline Al2 & $-C 7$ & $1.966(3)$ & C8 & -H81 & $0.98(3)$ \\
\hline Al2 & $-\mathrm{C} 8$ & $1.978(2)$ & $\mathrm{C} 8$ & $-\mathrm{H} 82$ & $0.90(3)$ \\
\hline 01 & $-\mathrm{C9}$ & $1.276(2)$ & $\mathrm{C} 8$ & $-\mathrm{H} 83$ & $0.94(3)$ \\
\hline 02 & $-\mathrm{C9}$ & $1.260(2)$ & C12 & $-\mathrm{H} 121$ & $0.91(2)$ \\
\hline C9 & $-\mathrm{C} 10$ & $1.514(3)$ & C14 & $-\mathrm{H} 141$ & $0.96(2)$ \\
\hline C10 & $-C 11$ & $1.432(2)$ & C17 & $-\mathrm{H} 171$ & $0.96(2)$ \\
\hline C10 & $-C 15$ & $1.409(3)$ & C17 & $-\mathrm{H} 172$ & $0.98(2)$ \\
\hline C11 & $-\mathrm{C} 12$ & $1.388(3)$ & C17 & $-\mathrm{H} 173$ & $0.94(2)$ \\
\hline C11 & - C16 & $1.548(3)$ & C18 & $-\mathrm{H} 181$ & $0.92(2)$ \\
\hline C12 & $-\mathrm{C} 13$ & $1.392(3)$ & C18 & $-\mathrm{H} 182$ & $0.98(2)$ \\
\hline C13 & $-C 14$ & $1.379(3)$ & C18 & $-\mathrm{H} 183$ & $0.91(2)$ \\
\hline C13 & $-\mathrm{C} 20$ & $1.544(3)$ & C19 & $-\mathrm{H} 191$ & $0.99(3)$ \\
\hline C14 & $-\mathrm{C} 15$ & $1.410(3)$ & C19 & $-\mathrm{H} 192$ & $0.97(2)$ \\
\hline C15 & - C24 & $1.552(2)$ & C19 & -H193 & $0.97(2)$ \\
\hline C16 & $-\mathrm{C} 17$ & $1.540(3)$ & C21 & $-\mathrm{H} 211$ & $1.04(4)$ \\
\hline C16 & $-\mathrm{C} 18$ & $1.540(3)$ & C21 & $-\mathrm{H} 212$ & $0.97(3)$ \\
\hline C16 & - C19 & $1.539(3)$ & C21 & $-\mathrm{H} 213$ & $0.95(3)$ \\
\hline $\mathrm{C} 20$ & $-C 21$ & $1.542(4)$ & $\mathrm{C} 22$ & $-\mathrm{H} 221$ & $0.96(3)$ \\
\hline C2O & $-\mathrm{C} 22$ & $1.523(4)$ & C22 & $-\mathrm{H} 222$ & $0.98(3)$ \\
\hline C2O & $-C 23$ & $1.526(3)$ & C22 & $-\mathrm{H} 223$ & $0.97(3)$ \\
\hline C24 & $-C 25$ & $1.534(3)$ & C23 & $-\mathrm{H} 231$ & $1.01(3)$ \\
\hline
\end{tabular}


Table S23 (cont.) - Bond Distances (Angstrom) for: Compound 9c

$\begin{array}{llllll}\mathrm{C} 24 & -\mathrm{C} 26 & 1.535(3) & \mathrm{C} 23 & -\mathrm{H} 232 & 0.99(3) \\ \mathrm{C} 24 & -\mathrm{C} 27 & 1.543(3) & \mathrm{C} 23 & -\mathrm{H} 233 & 0.97(2) \\ \mathrm{C} 1 & -\mathrm{H} 11 & 0.95(3) & \mathrm{C} 25 & -\mathrm{H} 251 & 0.97(2) \\ \mathrm{C} 1 & -\mathrm{H} 12 & 0.99(3) & \mathrm{C} 25 & -\mathrm{H} 252 & 0.97(2) \\ \mathrm{C} 1 & -\mathrm{H} 13 & 0.95(3) & \mathrm{C} 25 & -\mathrm{H} 253 & 0.92(2) \\ \mathrm{C} 2 & -\mathrm{H} 21 & 0.95(2) & \mathrm{C} 26 & -\mathrm{H} 261 & 0.97(3) \\ \mathrm{C} 2 & -\mathrm{H} 22 & 0.97(2) & \mathrm{C} 26 & -\mathrm{H} 262 & 0.93(3) \\ \mathrm{C} 2 & -\mathrm{H} 23 & 1.00(3) & \mathrm{C} 26 & -\mathrm{H} 263 & 0.95(3) \\ \mathrm{C} 3 & -\mathrm{H} 31 & 0.96(3) & \mathrm{C} 27 & -\mathrm{H} 271 & 1.02(3) \\ \mathrm{C} 3 & -\mathrm{H} 32 & 0.92(3) & \mathrm{C} 27 & -\mathrm{H} 272 & 0.97(2) \\ \mathrm{C} 3 & -\mathrm{H} 33 & 0.98(3) & \mathrm{C} 27 & -\mathrm{H} 273 & 0.94(3)\end{array}$

Table S24 - Bond Angles (Degrees) for: Compound 9c

\begin{tabular}{|c|c|c|c|c|c|c|c|}
\hline 01 & $-\mathrm{Nd}$ & $-\mathrm{C} 1$ & $85.40(6)$ & C21 & $-\mathrm{C} 20$ & $-C 23$ & $108.50(19)$ \\
\hline 01 & $-\mathrm{Nd}$ & $-\mathrm{C} 2$ & $164.58(6)$ & $\mathrm{C} 22$ & $-C 20$ & $-C 23$ & $109.50(19)$ \\
\hline 01 & $-\mathrm{Nd}$ & $-\mathrm{C5}$ & $105.20(6)$ & C15 & $-C 24$ & $-C 25$ & $116.04(16)$ \\
\hline 01 & $-\mathrm{Nd}$ & $-\mathrm{C} 6$ & $87.41(6)$ & C15 & $-C 24$ & $-\mathrm{C} 26$ & $109.98(15$ \\
\hline 01 & $-\mathrm{Nd}$ & $-02 \_a$ & $94.79(5)$ & C15 & $-C 24$ & $-C 27$ & $108.14(16$ \\
\hline C1 & $-\mathrm{Nd}$ & $-\mathrm{C} 2$ & $79.29(7)$ & $\mathrm{C} 25$ & $-C 24$ & $-\mathrm{C} 26$ & $107.93(17)$ \\
\hline C1 & $-\mathrm{Nd}$ & $-\mathrm{C} 5$ & $169.12(7)$ & $\mathrm{C} 25$ & $-C 24$ & $-\mathrm{C} 27$ & $105.22(17)$ \\
\hline C1 & $-\mathrm{Nd}$ & $-\mathrm{C} 6$ & $97.69(7)$ & $\mathrm{C} 26$ & $-C 24$ & $-C 27$ & $109.32(18)$ \\
\hline 02_a & $-\mathrm{Nd}$ & $-C 1$ & $99.13(6)$ & & & & \\
\hline $\mathrm{C} 2$ & $-\mathrm{Nd}$ & $-\mathrm{C5}$ & $90.03(7)$ & $\mathrm{Nd}$ & $-C 1$ & $-\mathrm{H} 11$ & $75.9(16)$ \\
\hline $\mathrm{C} 2$ & $-\mathrm{Nd}$ & $-\mathrm{C} 6$ & $92.77(7)$ & $\mathrm{Nd}$ & $-C 1$ & $-\mathrm{H} 12$ & $77.6(14)$ \\
\hline $02 \_a$ & $-\mathrm{Nd}$ & $-\mathrm{C} 2$ & $89.50(6)$ & $\mathrm{Nd}$ & $-\mathrm{C1}$ & $-\mathrm{H} 13$ & $177.8(15)$ \\
\hline $\mathrm{C} 5$ & $-\mathrm{Nd}$ & $-\mathrm{C} 6$ & $80.64(7)$ & Al1 & $-C 1$ & $-\mathrm{H} 11$ & $122.8(15)$ \\
\hline 02_a & $-\mathrm{Nd}$ & $-\mathrm{C} 5$ & $82.67(6)$ & Al1 & $-\mathrm{C1}$ & $-\mathrm{H} 12$ & $123.1(15)$ \\
\hline $02 \_a$ & $-\mathrm{Nd}$ & $-\mathrm{C} 6$ & $163.15(6)$ & Al1 & $-C 1$ & $-\mathrm{H} 13$ & $92.9(14)$ \\
\hline C1 & $-A 11$ & $-\mathrm{C} 2$ & $107.32(9)$ & H11 & $-C 1$ & $-\mathrm{H} 12$ & $105(2)$ \\
\hline C1 & $-A 11$ & $-\mathrm{C3}$ & $107.06(10)$ & H11 & $-C_{1}$ & $-\mathrm{H} 13$ & $105(2)$ \\
\hline C1 & $-A 11$ & $-\mathrm{C} 4$ & $107.65(11)$ & $\mathrm{H} 12$ & $-C 1$ & $-\mathrm{H} 13$ & $104(2)$ \\
\hline $\mathrm{C} 2$ & $-A 11$ & $-\mathrm{C3}$ & $106.14(11)$ & $\mathrm{Nd}$ & $-\mathrm{C} 2$ & $-\mathrm{H} 21$ & $77.2(14)$ \\
\hline C2 & $-A 11$ & $-\mathrm{C} 4$ & $110.69(11)$ & $\mathrm{Nd}$ & $-\mathrm{C} 2$ & $-\mathrm{H} 22$ & $177.0(16)$ \\
\hline C3 & $-A 11$ & $-\mathrm{C} 4$ & $117.53(12)$ & $\mathrm{Nd}$ & $-\mathrm{C} 2$ & $-\mathrm{H} 23$ & $79.1(15)$ \\
\hline C5 & $-A 12$ & $-\mathrm{C} 6$ & $109.44(9)$ & Al1 & $-\mathrm{C} 2$ & $-\mathrm{H} 21$ & $124.0(15)$ \\
\hline C5 & $-A 12$ & $-C 7$ & $105.20(10)$ & Al1 & $-\mathrm{C} 2$ & $-\mathrm{H} 22$ & $92.4(14)$ \\
\hline C5 & $-A 12$ & $-\mathrm{C} 8$ & $106.45(10)$ & Al1 & $-\mathrm{C} 2$ & $-\mathrm{H} 23$ & $119.9(14)$ \\
\hline C6 & $-A 12$ & $-C 7$ & $106.72(11)$ & $\mathrm{H} 21$ & $-\mathrm{C} 2$ & $-\mathrm{H} 22$ & $104(2)$ \\
\hline C6 & $-A 12$ & $-\mathrm{C} 8$ & $108.44(10)$ & $\mathrm{H} 21$ & $-\mathrm{C} 2$ & $-\mathrm{H} 23$ & $108(2)$ \\
\hline C7 & $-A 12$ & $-\mathrm{C} 8$ & $120.27(11)$ & $\mathrm{H} 22$ & $-\mathrm{C} 2$ & $-\mathrm{H} 23$ & $103(2)$ \\
\hline $\mathrm{Nd}$ & -01 & $-\mathrm{C9}$ & $175.41(13)$ & Al1 & $-\mathrm{C3}$ & $-H 31$ & $110.7(17)$ \\
\hline Nd_a & -02 & $-\mathrm{CO}$ & $136.93(13)$ & Al1 & $-\mathrm{C} 3$ & $-H 32$ & $108.7(19)$ \\
\hline $\mathrm{Nd}$ & $-C 1$ & $-A 11$ & $84.93(7)$ & Al1 & $-\mathrm{C3}$ & $-\mathrm{H} 33$ & $111.3(18)$ \\
\hline $\mathrm{Nd}$ & $-\mathrm{C} 2$ & $-A 11$ & $84.73(8)$ & H31 & $-\mathrm{C3}$ & $-\mathrm{H} 32$ & $111(3)$ \\
\hline $\mathrm{Nd}$ & $-\mathrm{C5}$ & $-A 12$ & $84.39(7)$ & H31 & $-\mathrm{C3}$ & $-\mathrm{H} 33$ & $106(2)$ \\
\hline $\mathrm{Nd}$ & $-\mathrm{C} 6$ & $-A 12$ & $84.26(8)$ & H32 & $-\mathrm{C3}$ & $-\mathrm{H} 33$ & $109(2)$ \\
\hline 01 & $-\mathrm{CO}$ & -02 & $121.12(18)$ & Al1 & $-\mathrm{C} 4$ & $-\mathrm{H} 41$ & $115.9(19)$ \\
\hline 01 & $-\mathrm{CO}$ & $-\mathrm{C} 10$ & $115.63(16)$ & Al1 & $-\mathrm{C} 4$ & $-\mathrm{H} 42$ & $113(2)$ \\
\hline 02 & $-\mathrm{CO}$ & $-\mathrm{C} 10$ & $123.23(17)$ & Al1 & $-\mathrm{C} 4$ & $-\mathrm{H} 43$ & $111.7(18)$ \\
\hline C9 & $-\mathrm{C} 10$ & $-C 11$ & $118.76(16)$ & H41 & $-\mathrm{C} 4$ & $-\mathrm{H} 42$ & $108(3)$ \\
\hline C9 & $-\mathrm{C} 10$ & $-\mathrm{C} 15$ & $120.25(16)$ & H41 & $-\mathrm{C} 4$ & $-\mathrm{H} 43$ & $102(2)$ \\
\hline C11 & - C10 & - C15 & $120.31(18)$ & $\mathrm{H} 42$ & $-\mathrm{C} 4$ & $-\mathrm{H} 43$ & $106(3)$ \\
\hline C10 & $-C 11$ & $-\mathrm{C} 12$ & $117.28(17)$ & $\mathrm{Nd}$ & $-\mathrm{C5}$ & $-H 51$ & $79.9(15)$ \\
\hline C10 & $-C 11$ & $-C 16$ & $123.93(17)$ & $\mathrm{Nd}$ & $-\mathrm{C5}$ & $-H 52$ & $175.7(16)$ \\
\hline C12 & $-C 11$ & - C16 & $118.67(16)$ & $\mathrm{Nd}$ & $-\mathrm{C5}$ & $-\mathrm{H} 53$ & $76.8(13)$ \\
\hline C11 & $-\mathrm{C} 12$ & $-\mathrm{C} 13$ & $123.80(18)$ & Al2 & $-\mathrm{C5}$ & $-\mathrm{H} 51$ & $124.2(16)$ \\
\hline C12 & $-\mathrm{C} 13$ & $-\mathrm{C} 14$ & $117.12(19)$ & Al2 & $-\mathrm{C5}$ & $-\mathrm{H} 52$ & $91.7(16)$ \\
\hline C12 & $-C 13$ & $-\mathrm{C} 20$ & $119.32(16)$ & Al2 & $-\mathrm{C5}$ & $-\mathrm{H} 53$ & $125.4(13)$ \\
\hline C14 & $-C 13$ & $-\mathrm{C} 20$ & $123.56(17)$ & H51 & $-\mathrm{C} 5$ & $-\mathrm{H} 52$ & $104(2)$ \\
\hline C13 & - C14 & - C15 & $123.02(19)$ & H51 & $-\mathrm{C5}$ & $-\mathrm{H} 53$ & $102(2)$ \\
\hline C10 & $-C 15$ & $-C 14$ & $117.82(16)$ & H52 & $-\mathrm{C5}$ & $-\mathrm{H} 53$ & $104(2)$ \\
\hline C10 & $-C 15$ & $-C 24$ & $127.01(17)$ & $\mathrm{Nd}$ & $-\mathrm{C} 6$ & $-H 61$ & $76.7(16)$ \\
\hline
\end{tabular}


Table 524 (cont.)

\begin{tabular}{|c|c|c|c|c|c|c|}
\hline C14 & $-C 15$ & $-C 24$ & $115.11(17)$ & $\mathrm{Nd}$ & $-\mathrm{C} 6$ & $-\mathrm{H} 62$ \\
\hline C11 & - C16 & $-\mathrm{C} 17$ & $108.58(16)$ & $\mathrm{Nd}$ & $-\mathrm{C} 6$ & $-\mathrm{H} 63$ \\
\hline C11 & - C16 & - C18 & $112.78(16)$ & Al2 & $-\mathrm{C} 6$ & $-\mathrm{H} 61$ \\
\hline C11 & - C16 & - C19 & $112.14(17)$ & Al2 & $-\mathrm{C6}$ & $-\mathrm{H} 62$ \\
\hline C17 & - C16 & - C18 & $111.00(18)$ & Al2 & $-\mathrm{C} 6$ & $-\mathrm{H} 63$ \\
\hline C17 & - C16 & - C19 & $106.52(17)$ & H61 & $-\mathrm{C} 6$ & $-\mathrm{H} 62$ \\
\hline C18 & - C16 & - C19 & $105.65(17)$ & H61 & $-\mathrm{C} 6$ & $-\mathrm{H} 63$ \\
\hline C13 & $-\mathrm{C} 20$ & $-C 21$ & $110.28(18)$ & $\mathrm{H} 62$ & $-\mathrm{C} 6$ & $-\mathrm{H} 63$ \\
\hline C13 & $-\mathrm{C} 20$ & $-\mathrm{C} 22$ & $112.49(18)$ & Al2 & $-\mathrm{C} 7$ & $-\mathrm{H} 71$ \\
\hline C13 & $-C 20$ & $-C 23$ & $108.49(19)$ & Al2 & $-C 7$ & $-\mathrm{H} 72$ \\
\hline C21 & $-\mathrm{C} 20$ & $-C 22$ & $107.5(2)$ & Al2 & $-\mathrm{C} 7$ & $-\mathrm{H} 73$ \\
\hline H71 & $-C 7$ & $-\mathrm{H} 72$ & $107(3)$ & $\mathrm{C} 20$ & $-\mathrm{C} 22$ & $-\mathrm{H} 2$ \\
\hline $\mathrm{H} 71$ & $-C 7$ & $-\mathrm{H} 73$ & $105(2)$ & $\mathrm{C} 20$ & $-\mathrm{C} 22$ & $-\mathrm{H} 2$ \\
\hline H72 & $-C 7$ & $-\mathrm{H} 73$ & $109(3)$ & $\mathrm{C} 20$ & $-\mathrm{C} 22$ & $-\mathrm{H} 2$ \\
\hline Al2 & $-\mathrm{C} 8$ & $-H 81$ & $113.1(14)$ & H221 & $-\mathrm{C} 22$ & $-\mathrm{H}_{2}$ \\
\hline Al2 & $-\mathrm{C} 8$ & $-\mathrm{H} 82$ & $110.3(17)$ & $\mathrm{H} 221$ & $-\mathrm{C} 22$ & $-\mathrm{H} 2$ \\
\hline Al2 & $-\mathrm{C} 8$ & $-\mathrm{H} 83$ & $112.7(16)$ & $\mathrm{H} 222$ & $-\mathrm{C} 22$ & $-\mathrm{H} 2$ \\
\hline H81 & $-\mathrm{C} 8$ & $-\mathrm{H} 82$ & $110(2)$ & $\mathrm{C} 20$ & $-\mathrm{C} 23$ & $-\mathrm{H} 2$ \\
\hline H81 & $-\mathrm{C} 8$ & $-\mathrm{H} 83$ & $104(2)$ & $\mathrm{C} 20$ & $-\mathrm{C} 23$ & $-\mathrm{H}$ \\
\hline H82 & $-\mathrm{C} 8$ & $-\mathrm{H} 83$ & $106(2)$ & $\mathrm{C} 20$ & $-\mathrm{C} 23$ & $-\mathrm{H} 2$ \\
\hline C11 & $-\mathrm{C} 12$ & $-\mathrm{H} 121$ & $118.8(13)$ & H231 & $-\mathrm{C} 23$ & $-\mathrm{H} 2$ \\
\hline C13 & $-\mathrm{C} 12$ & $-\mathrm{H} 121$ & $117.4(13)$ & H231 & $-C 23$ & $-\mathrm{H} 2$ \\
\hline C13 & - C14 & $-\mathrm{H} 141$ & $118.8(13)$ & $\mathrm{H} 232$ & $-\mathrm{C} 23$ & $-\mathrm{H}_{2}$ \\
\hline C15 & - C14 & $-\mathrm{H} 141$ & $118.2(13)$ & C24 & $-C 25$ & $-\mathrm{H} 2$ \\
\hline C16 & - C17 & $-\mathrm{H} 171$ & $112.8(12)$ & $\mathrm{C} 24$ & $-\mathrm{C} 25$ & $-\mathrm{H}_{2}^{2}$ \\
\hline C16 & $-\mathrm{C} 17$ & $-\mathrm{H} 172$ & $109.9(13)$ & $\mathrm{C} 24$ & $-C 25$ & $-\mathrm{H} 2$ \\
\hline C16 & $-\mathrm{C} 17$ & $-\mathrm{H} 173$ & $111.6(13)$ & $\mathrm{H} 251$ & $-C 25$ & $-\mathrm{H} 2$ \\
\hline H171 & - C17 & $-\mathrm{H} 172$ & $107.6(18)$ & H251 & $-C 25$ & $-\mathrm{H}_{2}$ \\
\hline H171 & $-\mathrm{C} 17$ & $-\mathrm{H} 173$ & $108.1(19)$ & H252 & $-C 25$ & $-\mathrm{H} 2$ \\
\hline H172 & - C17 & $-\mathrm{H} 173$ & $106.5(19)$ & C24 & $-C 26$ & $-\mathrm{H} 2$ \\
\hline C16 & - C18 & - H181 & $115.7(14)$ & $\mathrm{C} 24$ & $-\mathrm{C} 26$ & $-\mathrm{H} 2$ \\
\hline C16 & - C18 & $-\mathrm{H} 182$ & $109.7(13)$ & C24 & $-\mathrm{C} 26$ & $-\mathrm{H} 2$ \\
\hline C16 & $-\mathrm{C} 18$ & $-\mathrm{H} 183$ & $110.0(16)$ & H261 & $-C 26$ & $-\mathrm{H}_{2}$ \\
\hline H181 & - C18 & - H182 & $106.3(19)$ & H261 & $-\mathrm{C} 26$ & $-\mathrm{H}_{2}$ \\
\hline H181 & - C18 & - H183 & $107.0(19)$ & H262 & $-\mathrm{C} 26$ & $-\mathrm{H}_{2}$ \\
\hline H182 & - C18 & $-\mathrm{H} 183$ & $107.8(19)$ & C24 & $-\mathrm{C} 27$ & $-\mathrm{H}_{2}^{2}$ \\
\hline C16 & - C19 & - H191 & $111.5(15)$ & C24 & $-\mathrm{C} 27$ & $-\mathrm{H} 2$ \\
\hline C16 & - C19 & - H192 & $112.6(13)$ & C24 & $-\mathrm{C} 27$ & $-\mathrm{H} 2$ \\
\hline C16 & - C19 & $-\mathrm{H} 193$ & $108.1(15)$ & H271 & $-\mathrm{C} 27$ & $-\mathrm{H} 2$ \\
\hline H191 & - C19 & $-\mathrm{H} 192$ & $108(2)$ & $\mathrm{H} 271$ & $-\mathrm{C} 27$ & $-\mathrm{H} 2$ \\
\hline H191 & - C19 & -H193 & $108(2)$ & $\mathrm{H} 272$ & $-\mathrm{C} 27$ & $-\mathrm{H}_{2}$ \\
\hline H192 & - C19 & - H193 & $108.7(19)$ & & & \\
\hline C20 & $-C 21$ & $-\mathrm{H} 211$ & $110(2)$ & & & \\
\hline C2O & $-C 21$ & $-\mathrm{H} 212$ & $109.5(15)$ & & & \\
\hline C20 & $-C 21$ & $-H 213$ & $104(2)$ & & & \\
\hline H211 & $-C 21$ & $-\mathrm{H} 212$ & $109(3)$ & & & \\
\hline H211 & $-C 21$ & $-H 213$ & $118(3)$ & & & \\
\hline H212 & $-C 21$ & $-H 213$ & $105(3)$ & & & \\
\hline
\end{tabular}

Bond Angles (Degrees) for: Compound 9c

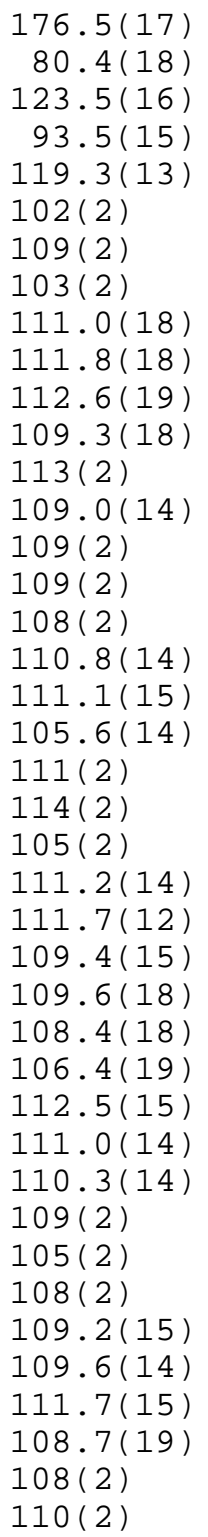

Translation of Symmetry Code to Equiv.Pos $a=[2555.00]=-x, y, 0.5-z$ 


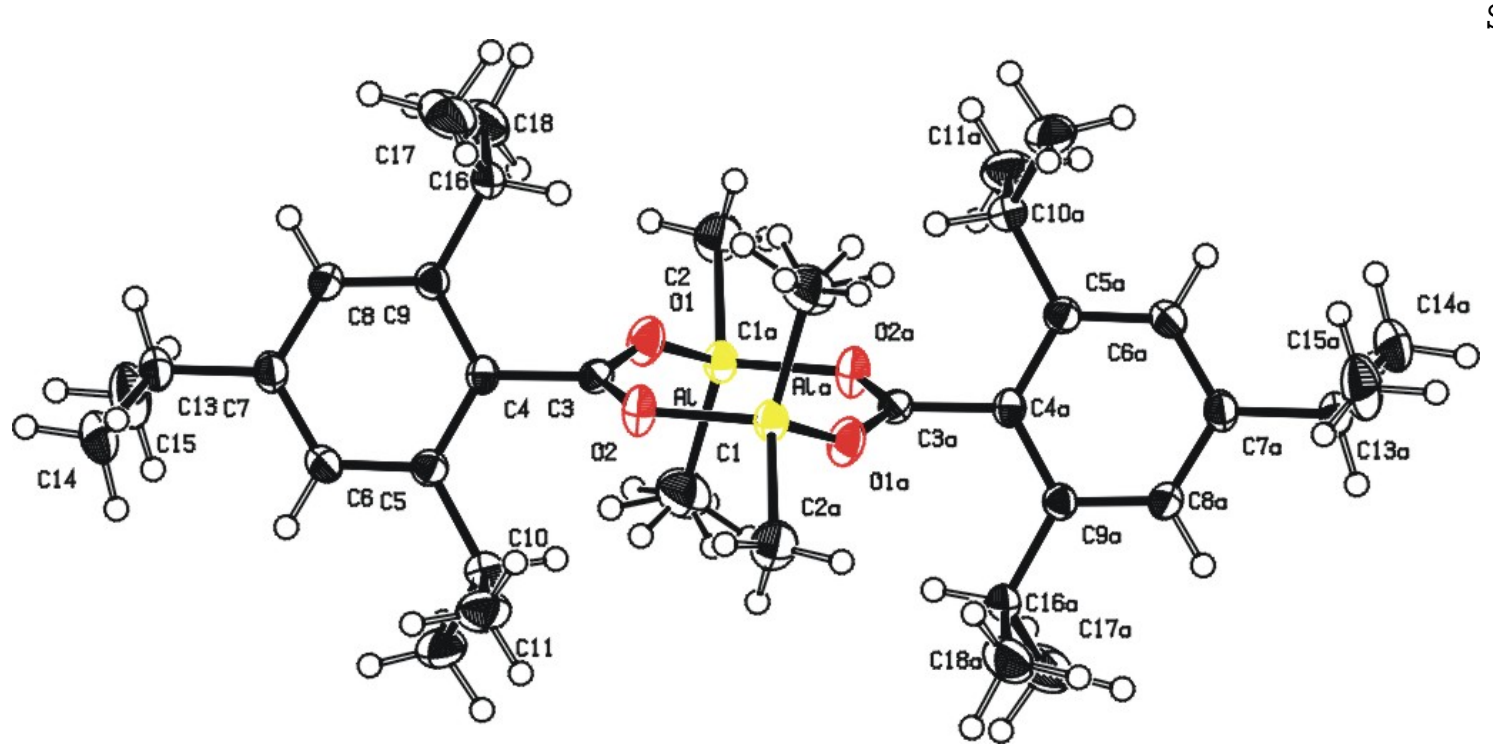

Molecular structure of $\left[\mathrm{Me}_{2} \mathrm{Al}\left(\mu-\mathrm{O}_{2} \mathrm{CAr}^{\mathrm{iPr}}\right)\right]_{2}(\mathbf{1 0 b})$

Table S25 - Crystal Data and Details of the Structure Determination for: Compound 10b

Crystal Data

Formula

Formula weight

Crystal System

space group

$a, b, c$ [Angstrom]

alpha, beta, gamma [deg]

$\mathrm{V}$ [Ang**3]

Z

$D($ calc $)\left[\mathrm{g} / \mathrm{cm}^{*} * 3\right]$

$\mathrm{Mu}($ MoKa) $[/ \mathrm{mm}]$

$\mathrm{F}(000)$

crystal size [mm]

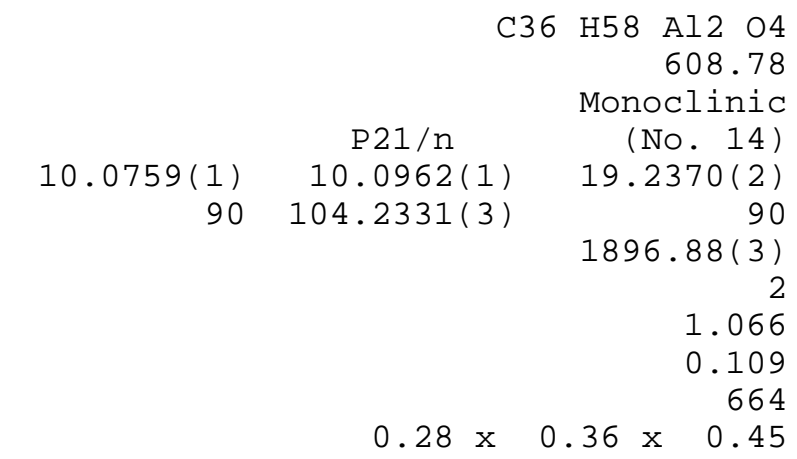

Data Collection

Temperature (K)

Radiation [Angstrom]

Theta Min-Max [Deg]

Moka 0.71073

Dataset

Tot., Uniq. Data, R(int)

observed data $[\mathrm{I}>2.0 \mathrm{sigma}(\mathrm{I})]$

$2.6, \quad 25.3$

$-12: 12 ;-12: 12 ;-23: 23$

41190, 3455, 0.040

Refinement

Nref, Npar

$3455, \quad 318$

$\mathrm{R}$, WR2, $\mathrm{S}$

$0.0348,0.0945,1.03$

$W=1 /\left[\backslash S^{\wedge} 2^{\wedge}\left(F O^{\wedge} 2^{\wedge}\right)+(0.0495 P)^{\wedge} 2^{\wedge}+\odot .7606 P\right]$ where $P=\left(F 0^{\wedge} 2^{\wedge}+2 F^{\wedge} C^{\wedge}\right) / 3$

Max. and Av. Shift/Error

Min. and Max. Resd. Dens. [e/Ang^3]

$0.00,0.00$

$-0.21,0.33$ 
Table S26 - Final Coordinates and Equivalent Isotropic Displacement Parameters of the non-Hydrogen atoms for: Compound 10b

\begin{tabular}{|c|c|c|c|c|}
\hline Atom & $x$ & $y$ & z & $\mathrm{U}(\mathrm{eq}) \quad[\mathrm{Ang} \wedge$ \\
\hline$-\cdots$ & -- & - & - & \\
\hline Al & $1.05809(4)$ & $0.15956(4)$ & $\odot .44289(2)$ & ๑. $0238(1)$ \\
\hline 01 & ๑.89481(9) & $0.10037(10)$ & $\odot .45372(5)$ & ๑. . $338(3)$ \\
\hline 02 & ๑.81514(9) & $-0.09211(9)$ & ๑.47955(5) & $0.0309(3)$ \\
\hline C1 & $1.0921(2)$ & $0.0783(2)$ & $0.35747(10)$ & $0.0519(7)$ \\
\hline $\mathrm{C} 2$ & $1.06346(18)$ & $0.34902(15)$ & $0.45847(11)$ & $0.0412(5)$ \\
\hline C3 & $0.80321(12)$ & 0.01233(12) & $0.44311(6)$ & $0.0214(3)$ \\
\hline C4 & $0.67450(12)$ & ๑. .03419(12) & $\odot .38616(6)$ & $0.0206(3)$ \\
\hline $\mathrm{C} 5$ & $0.64549(12)$ & $-0.04837(12)$ & ๑.32542(7) & $0.0234(3)$ \\
\hline C6 & $0.52724(13)$ & $-0.02195(13)$ & $\odot .27202(7)$ & $0.0270(4)$ \\
\hline C7 & $0.43917(13)$ & $0.08145(13)$ & $\odot .27771(7)$ & $0.0261(4)$ \\
\hline $\mathrm{C} 8$ & $\odot .46979(13)$ & $0.15893(13)$ & $\odot .33940(7)$ & $0.0249(4)$ \\
\hline $\mathrm{C9}$ & $0.58706(12)$ & $0.13807(12)$ & $0.39436(6)$ & $0.0219(3)$ \\
\hline C10 & $0.73810(14)$ & $-0.16414(13)$ & ๑.31761(7) & ○.0291(4) \\
\hline C11 & $0.6831(2)$ & $-0.29353(16)$ & $0.34082(10)$ & $\odot .0439(6)$ \\
\hline C12 & $0.75948(19)$ & $-0.17642(19)$ & $\odot .24204(9)$ & $0.0432(5)$ \\
\hline C13 & $0.31567(15)$ & $0.11433(15)$ & ๑.21690( (8) & $0.0346(4)$ \\
\hline C14 & $0.22573(17)$ & $-\odot .0 \odot 563(18)$ & $0.19043(10)$ & $\odot . \odot 440(5)$ \\
\hline C15 & $0.3623(2)$ & $0.1803(2)$ & $0.15559(10)$ & $0.0528(6)$ \\
\hline C16 & $0.61481(13)$ & $\odot .22564(14)$ & $0.46096(7)$ & $0.0269(4)$ \\
\hline C17 & $0.4931(2)$ & $0.2284(3)$ & $0.49486(11)$ & ๑. .0566( 7 \\
\hline C18 & $0.6537(2)$ & $0.36556(17)$ & $0.44332(10)$ & $0.0475(6$ \\
\hline
\end{tabular}

$U(e q)=1 / 3$ of the trace of the orthogonalized $U$ Tensor

Table S27 - Hydrogen Atom Positions and Isotropic Displacement Parameters for: Compound $\mathbf{1 0 b}$

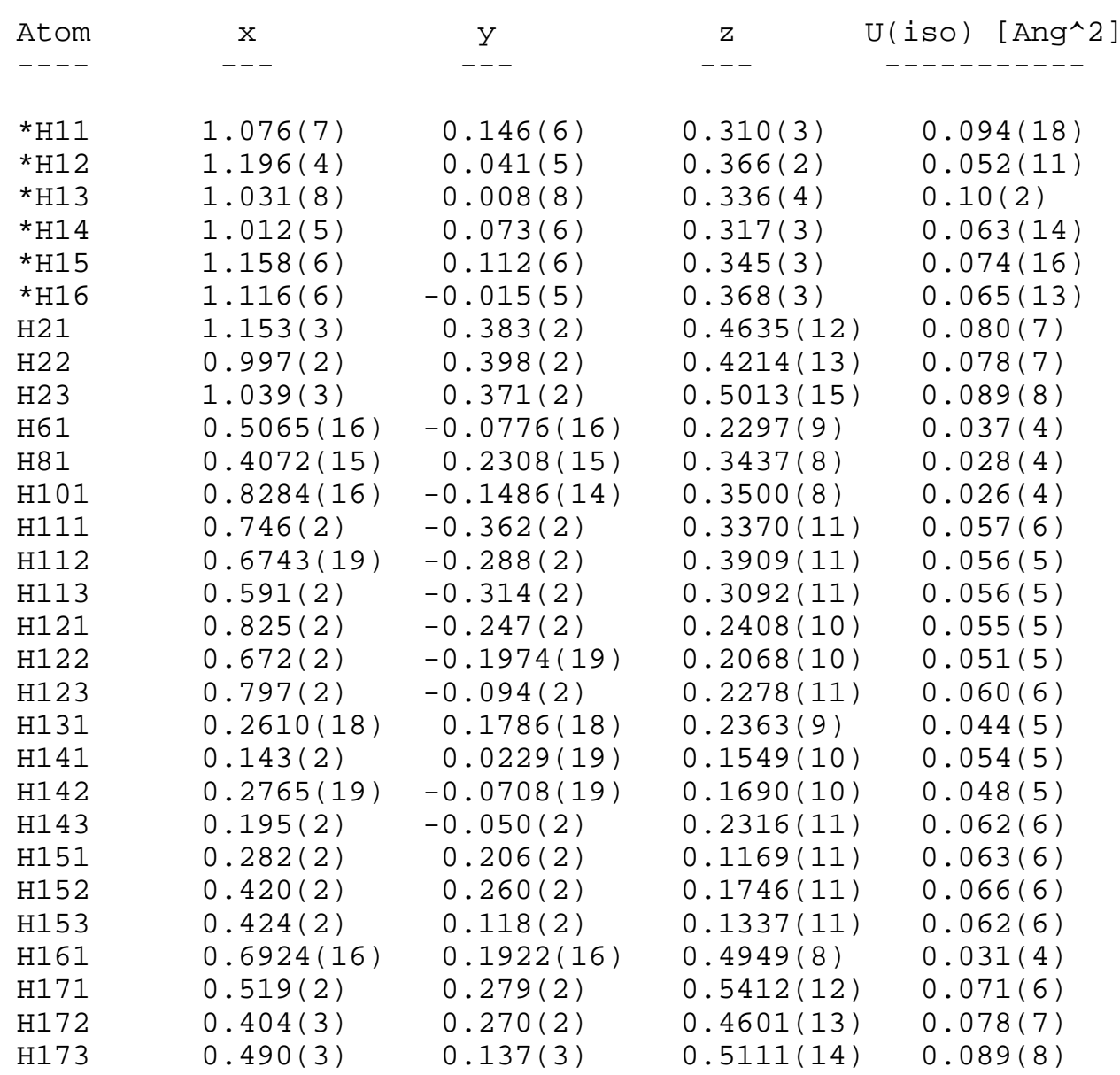


Table S27 (cont.) - Hydrogen Atom Positions and Isotropic Displacement Parameters for: Compound 10b

$\begin{array}{lcccc}\text { Atom } & \mathrm{x} & \mathrm{y} & \mathrm{z} & \mathrm{U}(\text { iso })[\text { Ang^2] } \\ \mathrm{H} & --- & --- & -- & \\ \text { H181 } & 0.669(2) & 0.422(2) & 0.4883(11) & 0.064(6) \\ \text { H182 } & 0.747(2) & 0.364(2) & 0.4218(12) & 0.075(7) \\ \text { H183 } & 0.579(2) & 0.409(2) & 0.4082(12) & 0.073(7)\end{array}$

The Temperature Factor has the Form of $\operatorname{Exp}(-\mathrm{T})$ Where $\mathrm{T}=8^{*}\left(\mathrm{Pi}{ }^{*} 2\right){ }^{*} \mathrm{U}^{*}(\operatorname{Sin}($ Theta $) /$ Lambda $) * * 2$ for Isotropic Atoms

Starred Atom sites have a S.O.F of 0.500

Table S28 - (An)isotropic Displacement Parameters for: Compound 10b

$\begin{array}{ccccccc}\text { Atom } & U(1,1) \text { or } U & U(2,2) & U(3,3) & U(2,3) & U(1,3) & U(1,2)\end{array}$

\begin{tabular}{|c|c|c|c|c|c|c|}
\hline $\mathrm{Al}$ & $\odot .0220(2)$ & $0.0228(2)$ & $0.0244(2)$ & $0.0041(2)$ & $0.0016(2)$ & $-0.0014(1)$ \\
\hline 01 & ๑.0194(5) & $\odot .0303(5)$ & $\odot .0479(6)$ & $\odot .0021(4)$ & $0.0009(4)$ & $-\odot .0031(4)$ \\
\hline 02 & $0.0223(5)$ & $\odot .0330(5)$ & $\odot .0328(5)$ & $\odot .0094(4)$ & $-0.0021(4)$ & $-0.0020(4$ \\
\hline C1 & $0.0558(12)$ & $0.0603(13)$ & $0.0403(10)$ & ) - $0.0095(9)$ & ๑.0132(9) & $0.0005(11)$ \\
\hline 2 & $0.0395(9)$ & $0.0261(8)$ & $0.0539(10)$ & $0.0025(7)$ & $0.0038(8)$ & $-0.0 \odot 29(7)$ \\
\hline c3 3 & o( 6$)$ & $0.0244(6)$ & & $-0.0023(5)$ & 0.0061 & $0.0014(5)$ \\
\hline C4 & $0.0181(6)$ & $\odot .0230(6)$ & $\odot .0203(6)$ & $0.0025(5)$ & $0.0041(5)$ & $-0.0013(5)$ \\
\hline C5 & $0.0230(6)$ & $0.0222(6)$ & ๑.0248(6) & $-0.0004(5)$ & $0.0054(5)$ & $-\odot .0002(5)$ \\
\hline $\mathrm{C} 6$ & $0.0290(7)$ & $\odot .0258(7)$ & $0.0235(6)$ & $-\odot .0043(5)$ & $0.0011(5)$ & $-\odot .00 \odot 6(5)$ \\
\hline $\mathrm{C} 7$ & $0.0228(6)$ & ๑.0266(7) & $0.0256(6)$ & $0.0005(5)$ & $-0.0006(5)$ & $-0.0011(5)$ \\
\hline $\mathrm{C} 8$ & $0.0204(6)$ & $0.0246(7)$ & ๑.0281(7) & $-0.0013(5)$ & $0.0027(5)$ & $0.0025(5)$ \\
\hline $\mathrm{Cg}$ & ๑.0193(6) & $0.0240(6)$ & ๑.0221(6) & $-\odot .0009(5)$ & $0.0043(5)$ & $-\odot .0012(5)$ \\
\hline C1C & $0.0296(7)$ & ๑.0281(7) & $0.0278(7)$ & $-0.0040(5)$ & $0.0038(6)$ & $0.0058(6)$ \\
\hline C11 & $7(11)$ & ๑.0268(8) & 0.6 & $-0.0022(7)$ & $0.0141(\varepsilon$ & $\odot .0048(7)$ \\
\hline C12 & $\odot .0479(10)$ & $0.0482(10)$ & $0.0361(8)$ & $-0.0052(7)$ & $0.0151(7)$ & ๑.0153(8) \\
\hline C13 & $0.0294(7)$ & $0.0338(8)$ & $0.0328(7)$ & $-0.0044(6)$ & $-0.0075(6)$ & $0.0040(6)$ \\
\hline C14 & $0.0309(8)$ & $0.0488(10)$ & $\odot .0438(9)$ & $-0.0081(8)$ & $-0.0070(7)$ & $-0.0052(7)$ \\
\hline C15 & $0.0544(11)$ & $0.0524(11)$ & $\odot .0390(9)$ & $0.0156(8)$ & $-0.0125(8$ & $-0.0049(9$ \\
\hline $\mathrm{C} 1$ & $0.0204(6)$ & $0.0333(7)$ & $0.0249(6)$ & $-0.0064(5)$ & $0.0016(5$ & $0.0031(5)$ \\
\hline $\mathrm{C} 1$ & $0.0426(10)$ & $0.0847(16)$ & 0.0 & $55(11)$ & $\odot .0219$ & $-\odot .0085(10)$ \\
\hline C1 & $0.0567(11)$ & $0.0343(9)$ & 0 & $-0.0113(7$ & $0.0000(8)$ & $-0.0051(8)$ \\
\hline
\end{tabular}

The Temperature Factor has the Form as given in Table S4

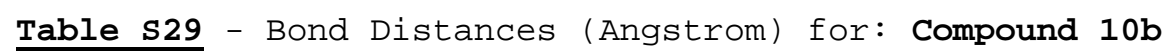

$\begin{array}{llllll}\text { Al } & -01 & 1.8099(10) & \mathrm{C} 1 & -\mathrm{H} 11 & 1.12(6) \\ \mathrm{Al} & -\mathrm{C} 1 & 1.941(2) & \mathrm{C} 1 & -\mathrm{H} 12 & 1.09(4) \\ \mathrm{Al} & -\mathrm{C} 2 & 1.9349(16) & \mathrm{C} 1 & -\mathrm{H} 13 & 0.96(8) \\ \mathrm{Al} & -02 \_\mathrm{a} & 1.8396(10) & \mathrm{C} 1 & -\mathrm{H} 14 & 0.98(5) \\ \text { 01 } & -\mathrm{C} 3 & 1.2613(16) & \mathrm{C} 1 & -\mathrm{H} 15 & 0.83(6) \\ 02 & -\mathrm{C} 3 & 1.2555(15) & \mathrm{C} 1 & -\mathrm{H} 16 & 0.98(5) \\ \mathrm{C} 3 & -\mathrm{C} 4 & 1.4940(17) & \mathrm{C} 2 & -\mathrm{H} 21 & 0.95(3) \\ \mathrm{C} 4 & -\mathrm{C} 5 & 1.4063(17) & \mathrm{C} 2 & -\mathrm{H} 22 & 0.98(2) \\ \mathrm{C} 4 & -\mathrm{C} 9 & 1.4034(17) & \mathrm{C} 2 & -\mathrm{H} 23 & 0.94(3) \\ \mathrm{C} 5 & -\mathrm{C} 6 & 1.3943(18) & \mathrm{C} 6 & -\mathrm{H} 61 & 0.969(17) \\ \mathrm{C} 5 & -\mathrm{C} 10 & 1.5259(18) & \mathrm{C} 8 & -\mathrm{H} 11 & 0.978(15) \\ \mathrm{C} 6 & -\mathrm{C} 7 & 1.3918(19) & \mathrm{C} 10 & -\mathrm{H} 101 & 0.981(16) \\ \mathrm{C} 7 & -\mathrm{C} 8 & 1.3912(18) & \mathrm{C} 11 & -\mathrm{H} 111 & 0.95(2)\end{array}$


Table S29 (cont.) - Bond Distances (Angstrom) for: Compound 10b

$\begin{array}{lll}\text { C7 } & - \text { C13 } & 1.520(2) \\ \text { C8 } & - \text { C9 } & 1.3943(18) \\ \text { C9 } & - \text { C16 } & 1.5248(18) \\ \text { C10 } & - \text { C11 } & 1.528(2) \\ \text { C10 } & - \text { C12 } & 1.526(2) \\ \text { C13 } & - \text { C14 } & 1.523(2) \\ \text { C13 } & - \text { C15 } & 1.526(2) \\ \text { C16 } & - \text { C17 } & 1.524(2) \\ \text { C16 } & - \text { C18 } & 1.526(2)\end{array}$

$\begin{array}{lll}\mathrm{C} 11 & -\mathrm{H} 112 & 0.99(2) \\ \mathrm{C} 11 & -\mathrm{H} 113 & 1.00(2) \\ \mathrm{C} 12 & -\mathrm{H} 121 & 0.98(2) \\ \mathrm{C} 12 & -\mathrm{H} 122 & 0.99(2) \\ \mathrm{C} 12 & -\mathrm{H} 123 & 0.98(2) \\ \mathrm{C} 13 & -\mathrm{H} 131 & 0.982(18) \\ \mathrm{C} 14 & -\mathrm{H} 141 & 0.98(2) \\ \mathrm{C} 14 & -\mathrm{H} 142 & 0.984(19) \\ \mathrm{C} 14 & -\mathrm{H} 143 & 1.02(2) \\ \mathrm{C} 15 & -\mathrm{H} 151 & 0.99(2) \\ \mathrm{C} 15 & -\mathrm{H} 152 & 1.01(2) \\ \mathrm{C} 15 & -\mathrm{H} 153 & 1.04(2) \\ \mathrm{C} 16 & -\mathrm{H} 161 & 0.949(16) \\ \mathrm{C} 17 & -\mathrm{H} 171 & 1.01(2) \\ \mathrm{C} 17 & -\mathrm{H} 172 & 1.07(3) \\ \mathrm{C} 17 & -\mathrm{H} 173 & 0.98(3) \\ \mathrm{C} 18 & -\mathrm{H} 181 & 1.02(2) \\ \mathrm{C} 18 & -\mathrm{H} 182 & 1.12(2) \\ \mathrm{C} 18 & -\mathrm{H} 183 & 0.98(2)\end{array}$

Table 530

\begin{tabular}{|c|c|c|c|}
\hline 01 & $-A l$ & $-C 1$ & $108.21(7)$ \\
\hline 01 & $-A l$ & $-\mathrm{C} 2$ & $107.47(7)$ \\
\hline 01 & $-A l$ & $-02 \_a$ & $104.71(5)$ \\
\hline C1 & $-A l$ & $-\mathrm{C} 2$ & $123.02(9)$ \\
\hline 02_a & $-A l$ & $-C 1$ & $107.35(7)$ \\
\hline 02_a & $-A l$ & $-\mathrm{C} 2$ & $104.65(7)$ \\
\hline $\mathrm{Al}$ & -01 & $-\mathrm{C3}$ & $149.74(9)$ \\
\hline Al_a & -02 & $-\mathrm{C3}$ & $135.71(9)$ \\
\hline 01 & $-\mathrm{C3}$ & -02 & $122.39(11)$ \\
\hline 01 & $-\mathrm{C3}$ & $-\mathrm{C} 4$ & $118.90(11)$ \\
\hline 02 & $-\mathrm{C} 3$ & $-\mathrm{C} 4$ & $118.70(11)$ \\
\hline C3 & $-\mathrm{C} 4$ & $-\mathrm{C5}$ & $119.17(11)$ \\
\hline C3 & $-\mathrm{C} 4$ & $-\mathrm{CO}$ & $118.93(10)$ \\
\hline $\mathrm{C} 5$ & $-\mathrm{C} 4$ & $-\mathrm{C9}$ & $121.90(11)$ \\
\hline C4 & $-\mathrm{C} 5$ & $-\mathrm{C} 6$ & $117.66(11)$ \\
\hline C4 & $-\mathrm{C} 5$ & - C10 & $121.91(11)$ \\
\hline C6 & $-\mathrm{C5}$ & - C10 & $120.43(11)$ \\
\hline $\mathrm{C} 5$ & $-\mathrm{C} 6$ & $-C 7$ & $122.10(12)$ \\
\hline C6 & $-C 7$ & $-\mathrm{C} 8$ & $118.48(12)$ \\
\hline C6 & $-C 7$ & $-C 13$ & $121.36(12)$ \\
\hline C8 & $-C 7$ & $-\mathrm{C} 13$ & $120.10(12)$ \\
\hline $\mathrm{C} 7$ & $-\mathrm{C} 8$ & $-\mathrm{C9}$ & $122.06(12)$ \\
\hline C4 & $-\mathrm{CO}$ & $-\mathrm{C8}$ & $117.78(11)$ \\
\hline C4 & $-\mathrm{C9}$ & - C16 & $122.50(11)$ \\
\hline $\mathrm{C8}$ & $-\mathrm{C9}$ & - C16 & $119.72(11)$ \\
\hline $\mathrm{C} 5$ & $-\mathrm{C} 10$ & $-C 11$ & $110.59(12)$ \\
\hline $\mathrm{C} 5$ & $-\mathrm{C} 10$ & $-\mathrm{C} 12$ & $112.89(12)$ \\
\hline C11 & - C10 & - C12 & $110.80(13)$ \\
\hline C7 & $-\mathrm{C} 13$ & - C14 & $113.00(13)$ \\
\hline C7 & $-C 13$ & - C15 & $109.92(13)$ \\
\hline C14 & $-C 13$ & $-C 15$ & $111.08(14)$ \\
\hline $\mathrm{CO}$ & - C16 & $-\mathrm{C} 17$ & $111.68(13)$ \\
\hline $\mathrm{C9}$ & - C16 & - C18 & $110.73(12)$ \\
\hline C17 & - C16 & - C18 & $110.84(16)$ \\
\hline Al & $-C 1$ & $-\mathrm{H} 11$ & $114(3)$ \\
\hline Al & $-\mathrm{C1}$ & $-\mathrm{H} 12$ & $113(2)$ \\
\hline $\mathrm{Al}$ & $-C 1$ & $-\mathrm{H} 13$ & $117(5)$ \\
\hline Al & $-C 1$ & $-\mathrm{H} 14$ & $115(3)$ \\
\hline Al & $-C 1$ & $-\mathrm{H} 15$ & $113(4)$ \\
\hline Al & $-C 1$ & - H16 & $109(3)$ \\
\hline
\end{tabular}

$\begin{array}{llll}\mathrm{C} 5 & -\mathrm{C} 6 & -\mathrm{H} 11 & 118.5(10) \\ \mathrm{C} 7 & -\mathrm{C} 6 & -\mathrm{H} 11 & 119.4(10) \\ \mathrm{C} 7 & -\mathrm{C} 8 & -\mathrm{H} 11 & 118.5(9) \\ \mathrm{C} 9 & -\mathrm{C} 8 & -\mathrm{H} 11 & 119.5(9) \\ \mathrm{C} 5 & -\mathrm{C} 10 & -\mathrm{H} 101 & 108.0(9) \\ \mathrm{C} 11 & -\mathrm{C} 10 & -\mathrm{H} 101 & 107.3(8) \\ \mathrm{C} 12 & -\mathrm{C} 10 & -\mathrm{H} 101 & 107.0(9) \\ \mathrm{C} 10 & -\mathrm{C} 11 & -\mathrm{H} 111 & 107.6(12) \\ \mathrm{C} 10 & -\mathrm{C} 11 & -\mathrm{H} 112 & 111.2(12) \\ \mathrm{C} 10 & -\mathrm{C} 11 & -\mathrm{H} 113 & 110.4(12) \\ \mathrm{H} 111 & -\mathrm{C} 11 & -\mathrm{H} 112 & 109.7(17) \\ \mathrm{H} 111 & -\mathrm{C} 11 & -\mathrm{H} 113 & 109.8(17) \\ \mathrm{H} 112 & -\mathrm{C} 11 & -\mathrm{H} 113 & 108.2(17) \\ \mathrm{C} 10 & -\mathrm{C} 12 & -\mathrm{H} 121 & 109.7(11) \\ \mathrm{C} 10 & -\mathrm{C} 12 & -\mathrm{H} 122 & 111.0(12) \\ \mathrm{C} 10 & -\mathrm{C} 12 & -\mathrm{H} 123 & 110.6(12) \\ \mathrm{H} 121 & -\mathrm{C} 12 & -\mathrm{H} 122 & 108.6(16) \\ \mathrm{H} 121 & -\mathrm{C} 12 & -\mathrm{H} 123 & 107.7(17) \\ \mathrm{H} 122 & -\mathrm{C} 12 & -\mathrm{H} 123 & 109.2(16) \\ \mathrm{C} 7 & -\mathrm{C} 13 & -\mathrm{H} 131 & 106.3(10) \\ \mathrm{C} 14 & -\mathrm{C} 13 & -\mathrm{H} 131 & 108.0(11) \\ \mathrm{C} 15 & -\mathrm{C} 13 & -\mathrm{H} 131 & 108.3(10) \\ \mathrm{C} 13 & -\mathrm{C} 14 & -\mathrm{H} 141 & 109.4(11) \\ \mathrm{C} 13 & -\mathrm{C} 14 & -\mathrm{H} 142 & 110.1(11) \\ \mathrm{C} 13 & -\mathrm{C} 14 & -\mathrm{H} 143 & 111.0(12) \\ \mathrm{H} 141 & -\mathrm{C} 14 & -\mathrm{H} 142 & 110.1(16) \\ \mathrm{H} 141 & -\mathrm{C} 14 & -\mathrm{H} 143 & 107.5(17) \\ \mathrm{H} 142 & -\mathrm{C} 14 & -\mathrm{H} 143 & 108.6(16) \\ \mathrm{C} 13 & -\mathrm{C} 15 & -\mathrm{H} 151 & 110.2(12) \\ \mathrm{C} 13 & -\mathrm{C} 15 & -\mathrm{H} 152 & 109.2(12) \\ \mathrm{C} 13 & -\mathrm{C} 15 & -\mathrm{H} 153 & 112.1(11) \\ \mathrm{H} 151 & -\mathrm{C} 15 & -\mathrm{H} 152 & 110.8(17) \\ \mathrm{H} 151 & -\mathrm{C} 15 & -\mathrm{H} 153 & 108.2(17) \\ \mathrm{H} 152 & -\mathrm{C} 15 & -\mathrm{H} 153 & 106.3(16) \\ \mathrm{C} 9 & -\mathrm{C} 16 & -\mathrm{H} 161 & 108.8(10) \\ \mathrm{C} 17 & -\mathrm{C} 16 & -\mathrm{H} 161 & 109.0(10) \\ \mathrm{C} 18 & -\mathrm{C} 16 & -\mathrm{H} 161 & 105.6(10) \\ \mathrm{C} 16 & -\mathrm{C} 17 & -\mathrm{H} 171 & 109.2(12) \\ \mathrm{C} 16 & -\mathrm{C} 17 & -\mathrm{H} 172 & 112.4(15) \\ \mathrm{C} 16 & -\mathrm{C} 17 & -\mathrm{H} 173 & 102.2(18) \\ \mathrm{H} 171 & -\mathrm{C} 17 & -\mathrm{H} 172 & 110.6(17)\end{array}$


Table S30 (cont.) - Bond Angles (Degrees) for: Compound 10b

$\begin{array}{llllllll}\mathrm{H} 11 & -\mathrm{C} 1 & -\mathrm{H} 12 & 106(4) & \mathrm{H} 171 & -\mathrm{C} 17 & -\mathrm{H} 173 & 103(2) \\ \mathrm{H} 11 & -\mathrm{C} 1 & -\mathrm{H} 13 & 99(6) & \mathrm{H} 172 & -\mathrm{C} 17 & -\mathrm{H} 173 & 119(2) \\ \mathrm{H} 12 & -\mathrm{C} 1 & -\mathrm{H} 13 & 107(6) & \mathrm{C} 16 & -\mathrm{C} 18 & -\mathrm{H} 181 & 108.9(11) \\ \mathrm{H} 14 & -\mathrm{C} 1 & -\mathrm{H} 15 & 111(5) & \mathrm{C} 16 & -\mathrm{C} 18 & -\mathrm{H} 182 & 110.8(10) \\ \mathrm{H} 14 & -\mathrm{C} 1 & -\mathrm{H} 16 & 103(5) & \mathrm{C} 16 & -\mathrm{C} 18 & -\mathrm{H} 183 & 111.8(12) \\ \mathrm{H} 15 & -\mathrm{C} 1 & -\mathrm{H} 16 & 106(5) & \mathrm{H} 181 & -\mathrm{C} 18 & -\mathrm{H} 182 & 111.0(16) \\ \mathrm{A} 1 & -\mathrm{C} 2 & -\mathrm{H} 21 & 111.3(13) & \mathrm{H} 181 & -\mathrm{C} 18 & -\mathrm{H} 183 & 105.4(17) \\ \mathrm{A} 1 & -\mathrm{C} 2 & -\mathrm{H} 22 & 113.4(12) & \mathrm{H} 182 & -\mathrm{C} 18 & -\mathrm{H} 183 & 108.9(17) \\ \mathrm{A} 1 & -\mathrm{C} 2 & -\mathrm{H} 23 & 111.4(13) & & & & \\ \mathrm{H} 21 & -\mathrm{C} 2 & -\mathrm{H} 22 & 110.7(18) & & & & \\ \mathrm{H} 21 & -\mathrm{C} 2 & -\mathrm{H} 23 & 106(2) & & & & \\ \mathrm{H} 22 & -\mathrm{C} 2 & -\mathrm{H} 23 & 104(2) & & & & \end{array}$

Translation of Symmetry Code to Equiv.Pos

$a=[3756.00]=2-x, y, 1.5-z$

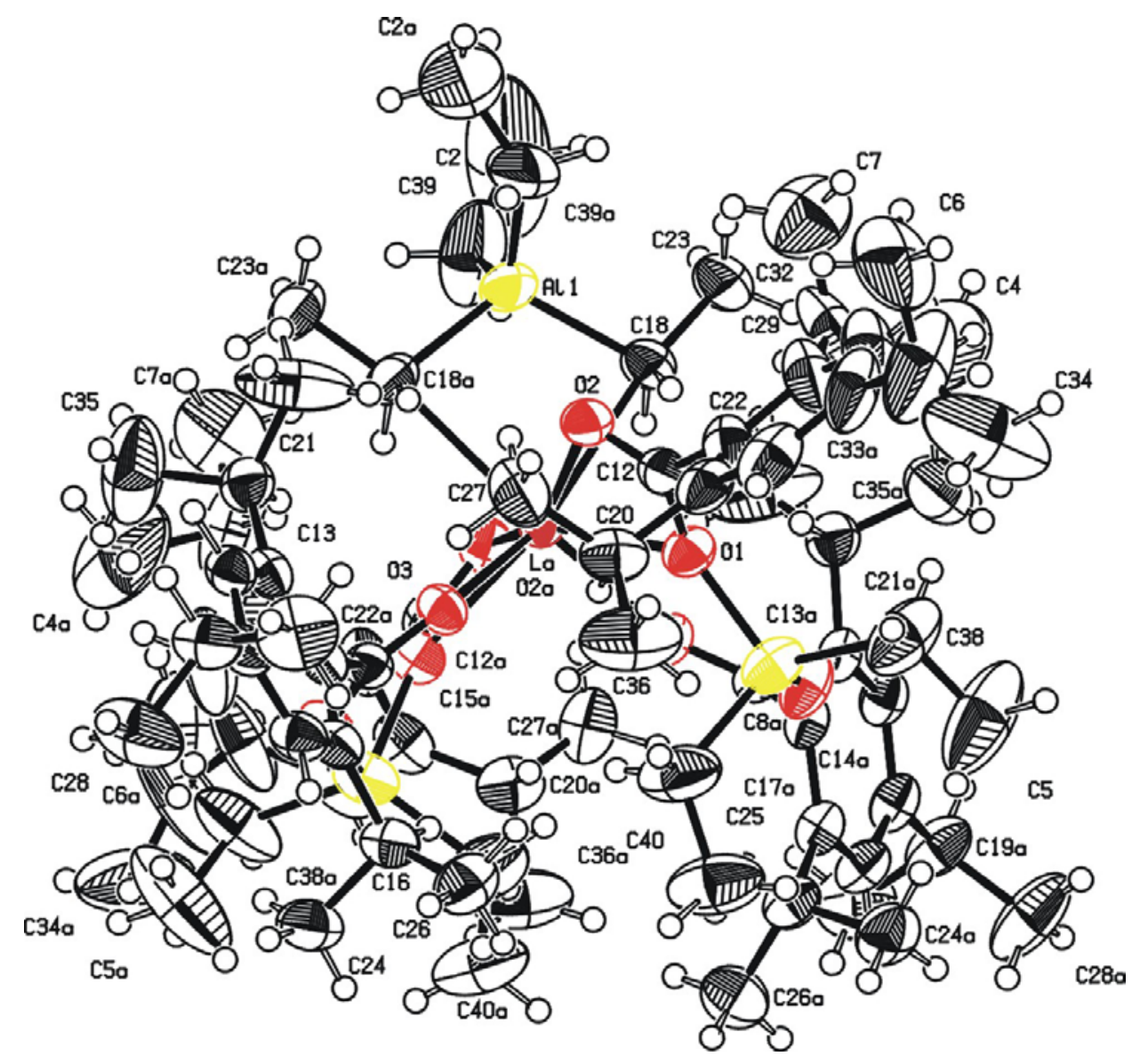

Molecular structure of $\left[\operatorname{LaAl}_{3} \mathrm{Et}_{8}\left(\mathrm{O}_{2} \mathrm{CC}_{6} \mathrm{H}_{2}{ }^{i} \mathrm{Pr}_{3}-2,4,6\right)_{4}\right.$ (7a) 